\title{
Anatomy of the inert two-Higgs-doublet model in the light of the LHC and non-LHC dark matter searches
}

\author{
Alexander Belyaev, ${ }^{1,2}$ Giacomo Cacciapaglia, ${ }^{3}$ Igor P. Ivanov, ${ }^{4}$ Felipe Rojas-Abatte, ${ }^{2,5}$ and Marc Thomas ${ }^{1,2}$ \\ ${ }^{1}$ Rutherford Appleton Laboratory, Didcot, United Kingdom \\ ${ }^{2}$ University of Southampton, Southampton, United Kingdom \\ ${ }^{3}$ Université Lyon, Université Lyon 1, CNRS/IN2P3, IPNL, F-69622 Villeurbanne, France \\ ${ }^{4}$ CFTP, Instituto Superior Técnico, Universidade de Lisboa, Lisbon 1049-001, Portugal \\ ${ }^{5}$ Universidad Técnica Federico Santa María, Valparaíso, Chile
}

(Received 15 March 2017; published 15 February 2018)

\begin{abstract}
The inert two-Higgs-doublet model (i2HDM) is a theoretically well-motivated example of a minimal consistent dark matter (DM) model which provides monojet, mono-Z, mono-Higgs, and vector-bosonfusion $+\boldsymbol{E}_{\mathrm{T}}^{\text {miss }}$ signatures at the LHC, complemented by signals in direct and indirect DM search experiments. In this paper we have performed a detailed analysis of the constraints in the full fivedimensional parameter space of the i2HDM, coming from perturbativity, unitarity, electroweak precision data, Higgs data from the LHC, DM relic density, direct/indirect DM detection, and LHC monojet analysis, as well as implications of experimental LHC studies on disappearing charged tracks relevant to a high DM mass region. We demonstrate the complementarity of the above constraints and present projections for future LHC data and direct DM detection experiments to probe further i2HDM parameter space. The model is implemented into the CALCHEP and MICROMEGAs packages, which are publicly available at the HEPMDB database, and it is ready for a further exploration in the context of the LHC, relic density, and DM direct detection.
\end{abstract}

DOI: 10.1103/PhysRevD.97.035011

\section{INTRODUCTION}

The evidence for dark matter (DM) is well established from several independent cosmological observations, including galactic rotation curves, cosmic microwave background fits of the WMAP and PLANCK data, gravitational lensing, large scale structure of the Universe, as well as interacting galaxy clusters such as the Bullet Cluster. Despite these large-scale evidences, the microscopic nature of the DM particles remains unknown, since no experiment so far has been able to claim their detection in the laboratory and probe their properties. Potentially, DM can be produced at the LHC and probed in the DM direct detection (DD) underground experiments. The fundamental importance and vast experimental opportunities make the search for and investigation of DM one of the key goals in astroparticle physics and high energy physics (HEP), worthy of the intense efforts undertaken by the physics community.

Published by the American Physical Society under the terms of the Creative Commons Attribution 4.0 International license. Further distribution of this work must maintain attribution to the author(s) and the published article's title, journal citation, and DOI. Funded by SCOAP .
At the other end of the length scale, the Standard Model (SM) of particle physics recently demonstrated its vitality once again. The scalar boson with mass $m_{H} \approx 125 \mathrm{GeV}$ found at the LHC [1,2] closely resembles, in all its manifestations, the SM Higgs boson. Since the SM cannot be the ultimate theory, many constructions beyond the SM (BSM) have been put forth, at different levels of sophistication. Yet, without direct experimental confirmation, none of them can be named the true theory beyond the SM.

One way the particle theory community can respond to this situation is to propose simple, fully calculable, renormalizable BSM models with viable DM candidates. We do not know yet which of these models (if any) corresponds to reality, but all models of this kind offer an excellent opportunity to gain insight into the intricate interplay among various astrophysical and collider constraints. We call here these models minimal consistent dark matter (MCDM) models. MCDM models which can be viewed as toy models, are self-consistent and can easily be incorporated into a bigger BSM model. Because of these attractive features, MCDM models can be considered as the next step beyond DM effective field theory (EFT) (see e.g. [3-15]) and simplified DM models (see e.g. [16-23]).

In this paper, we explore, in the light of the recent collider, astroparticle, and DD DM experimental data, the inert two-Higgs-doublet model (i2HDM), also known as 
the inert doublet model. This model is easily doable with analytic calculations, its parameter space is relatively small, and it can be strongly constrained by the present and future data. The model leads to a variety of collider signatures, and, in spite of many years of investigation, not all of them have yet been fully and properly explored. It is the goal of the present paper to investigate in fine detail the present constraints and the impact of the future LHC and DD DM data on the parameter space of this model.

The i2HDM [24-27] is a minimalistic extension of the SM with a second scalar doublet $\phi_{2}$ possessing the same quantum numbers as the SM Higgs doublet $\phi_{1}$ but with no direct coupling to fermions (the inert doublet). This construction is protected by the discrete $Z_{2}$ symmetry under which $\phi_{2}$ is odd and all the other fields are even. The scalar Lagrangian is

$$
\mathcal{L}=\left|D_{\mu} \phi_{1}\right|^{2}+\left|D_{\mu} \phi_{2}\right|^{2}-V\left(\phi_{1}, \phi_{2}\right)
$$

with the potential $V$ containing all scalar interactions compatible with the $Z_{2}$ symmetry,

$$
\begin{aligned}
V= & -m_{1}^{2}\left(\phi_{1}^{\dagger} \phi_{1}\right)-m_{2}^{2}\left(\phi_{2}^{\dagger} \phi_{2}\right)+\lambda_{1}\left(\phi_{1}^{\dagger} \phi_{1}\right)^{2}+\lambda_{2}\left(\phi_{2}^{\dagger} \phi_{2}\right)^{2} \\
& +\lambda_{3}\left(\phi_{1}^{\dagger} \phi_{1}\right)\left(\phi_{2}^{\dagger} \phi_{2}\right)+\lambda_{4}\left(\phi_{2}^{\dagger} \phi_{1}\right)\left(\phi_{1}^{\dagger} \phi_{2}\right) \\
& +\frac{\lambda_{5}}{2}\left[\left(\phi_{1}^{\dagger} \phi_{2}\right)^{2}+\left(\phi_{2}^{\dagger} \phi_{1}\right)^{2}\right] .
\end{aligned}
$$

All free parameters here are real, ${ }^{1}$ which precludes the $C P$ violation in the scalar sector. There is a large part of the parameter space in which only the first, SM-like doublet acquires the vacuum expectation value (VEV). In the notation $\left\langle\phi_{i}^{0}\right\rangle=v_{i} / \sqrt{2}$, this inert minimum corresponds to $v_{1}=v, v_{2}=0$. In the unitary gauge, the doublets are expanded near the minimum as

$$
\phi_{1}=\frac{1}{\sqrt{2}}\left(\begin{array}{c}
0 \\
v+H
\end{array}\right), \quad \phi_{2}=\frac{1}{\sqrt{2}}\left(\begin{array}{c}
\sqrt{2} h^{+} \\
h_{1}+i h_{2}
\end{array}\right) .
$$

The $Z_{2}$ symmetry is still conserved by the vacuum state, which forbids direct coupling of any single inert field to the SM fields, and it stabilizes the lightest inert boson against decay. Pairwise interactions of the inert scalars with the gauge bosons and with the SM-like Higgs $H$ are still possible, which gives rise to various i2HDM signatures at colliders and in the DM detection experiments.

The idea that the symmetry-protected second Higgs doublet naturally produces a scalar dark matter candidate was first mentioned more that 30 years ago [24]. However, the real interest in phenomenological consequences of the i2HDM woke up in mid-2000 and intensified in the past

\footnotetext{
${ }^{1}$ Even if we started with a complex $\lambda_{5}$, we could redefine the second doublet via a global phase rotation, which would render $\lambda_{5}$ real without affecting any other part of the Lagrangian.
}

few years. Its simplicity, predictive power, rich yet manageable parameter space, makes it an ideal playground for checking its compatibility with the DM relic density, with the results of the direct and indirect DM searches, and with collider searches of various BSM signals.

Assuming that the lightest inert scalar is the only DM candidate, one typically finds that the low-mass region, below about $50 \mathrm{GeV}$, is excluded by the relic density constraints coupled with the LHC constraints on the invisible Higgs decay [28-30]. The funnel region, with the DM mass close to $M_{H} / 2$, the intermediate, 100$500 \mathrm{GeV}$, and the high mass regions are still compatible with data and lead to interesting predictions at colliders. Additional theoretical constraints on the parameter space and DM candidate properties can be deduced from assumptions of full stability of the i2HDM up to the PLANCK scale [31,32] or of multidoublet Higgs inflation [33]. The i2HDM can also produce signals for direct [34] and indirect DM search experiments via heavy inert scalar annihilation, which can be detectable via $\gamma$ rays [35-37] or via its neutrino $[38,39]$ and cosmic-ray signals [40].

The i2HDM can also have interesting cosmological consequences. Being an example of 2HDM, it possesses a rich vacuum structure, which evolves at high temperatures [41-43]. This opens up the possibility within i2HDM that the early Universe, while cooling down, went through a sequence of phase transitions including strong first-order phase transitions [44-50]. Such analyses are capable of restricting the parameter space; for example, the recent study [50] showed that combining the strong firstorder phase transition with other astroparticle and collider constraints gives preference to the funnel region.

There has also been a number of studies on collider signatures of the i2HDM. They focus on specific processes such as SM-like Higgs decays to $\gamma \gamma$ and $\gamma Z$ [28,51-53], multilepton production plus missing transverse momentum $\left(\boldsymbol{E}_{\mathrm{T}}^{\text {miss }}\right)$ [54-56] with as many as five leptons [57], dijet + $\boldsymbol{E}_{\mathrm{T}}^{\text {miss }}$ [58], and dileptons accompanied with dijets [56]. Other works present combined analyses of astroparticle and collider constraints [29,57,59-62]. Comparing the i2HDM predictions with the electroweak precision data, the measured SM-like Higgs properties, the nonobservation of long-lived charged particles and other exotic signals, and finally the astroparticle observations, allows one to significantly restrict the i2HDM parameter space. The recent analysis [29] gave a detailed account of these constraints. For specific benchmark points or benchmark planes in the surviving parameter space, it predicted the cross section of pair production of inert scalars followed by various modes of their decay. As for the specific signatures of the i2HDM at the LHC, dileptons and mono- $Z$ signals were mentioned. An earlier analysis [60] investigated multilepton, multijet, mono- $Z$, and several channels for the monojet with large $\boldsymbol{E}_{\mathrm{T}}^{\text {miss }}$. Reference [59] took into account one-loop corrections to the masses and, for a part of the numerical scans, 
included the additional theoretical constraint that the perturbativity and stability be satisfied up to a large scale $\Lambda$. The version of i2HDM equipped with Peccei-Quinn $U(1)$ symmetry spontaneously broken to $Z_{2}$ was investigated in [62]. Here, dark matter acquires a second component, the axion, which changes the DM phenomenology. It is also possible to hunt for i2HDM at the future colliders, via searching for new scalars and reconstructing the potential [63] or by accurately measuring the SM-like Higgs couplings and deducing patterns of the deviations from the SM [64].

In the present work, to these many studies on the i2HDM, we add the following:

(i) detailed combined analysis of the 2 2HDM model in its full five-dimensional (5D) parameter space, taking into account perturbativity and unitarity, LEP and electroweak precision data, Higgs data from the LHC, DM relic density, direct/indirect DM detection complemented by realistic (beyond-theparton-level) LHC monojet analysis at the LHC;

(ii) quantitative exploration of the surviving regions of parameters, including very fine details and a qualitatively new region not seen in previous studies, which is enabled by our extensive numerical scans;

(iii) a combination of different processes giving the LHC monojet signatures: those with direct DM pair production and those with associate production of DM with another scalar with a close mass from the inert multiplet;

(iv) implication of experimental LHC studies on disappearing charged tracks relevant to a high $(\simeq 500 \mathrm{GeV}) \mathrm{DM}$ mass region;

(v) separate, equally detailed analyses for the assumptions of the DM relic density being fitted to the PLANCK results or underabundant, allowing thus for additional allowed regions of the parameter space.

All these points above are in close focus of the present paper where we have performed a comprehensive scan and study of the full parameter space of the i2HDM model. In addition, we have performed an independent implementation and validation of the model in two gauges including Higgs-gluon-gluon and Higgs-photon-photon effective couplings, and we made it public together with the LanHEP model source.

The paper is organized as follows. In Sec. II we discuss the i2HDM model parameter space, implementation, theoretical constraints, as well as constraints from LEP and electroweak precision data. In Sec. III we discuss results of a comprehensive scan of the i2HDM parameter space and combined constraints considering both the cases when the relic density is "just right" and agrees with the PLANCK results and when it is underabundant. In this section we also present the reach of LHC studies in the high DM mass region using results on disappearing charged tracks. In Sec. IV we present results on future projections of the LHC and DM DD experiments in combination with all previous constraints. Finally, in Sec. V we draw our conclusions.

\section{II. i2HDM: PARAMETER SPACE, MODEL IMPLEMENTATION, THEORETICAL AND EXPERIMENTAL CONSTRAINTS}

\section{A. Constraints from the Higgs potential}

In order to represent a viable model, the potential (2) must be bounded from below and must have a neutral, not charge-breaking, vacuum. The former requirement leads to the well-known restrictions on the free parameters of the model,

$\lambda_{1}>0, \quad \lambda_{2}>0, \quad 2 \sqrt{\lambda_{1} \lambda_{2}}+\lambda_{3}>0$,

$2 \sqrt{\lambda_{1} \lambda_{2}}+\lambda_{3}+\lambda_{4}-\left|\lambda_{5}\right|>0$.

The absence of the charge-breaking vacuum is guaranteed if one assumes

$$
\lambda_{4}-\left|\lambda_{5}\right|<0
$$

This is a sufficient but not necessary condition for the vacuum to be neutral. A neutral vacuum can also be achieved for positive $\lambda_{4}-\left|\lambda_{5}\right|$ with appropriate $m_{1}^{2}$ and $m_{2}^{2}$. However, in this case the lightest DM candidate will be the charged scalar. Condition (5) avoids this situation as well.

Once these restrictions are applied, the vacuum is neutral, and one can calculate the masses of the physical Higgs bosons. In addition to the SM-like scalar $H$, one gets charged $h^{ \pm}$and neutral $h_{1}, h_{2}$ scalars. It is well known that the two neutral scalars of the i2HDM have opposite $C P$ parities, but it is impossible to unambiguously assign which of them is $C P$ even and which is $C P$ odd. In the absence of any suitable vertex, the model has two $C P$ symmetries, $h_{1} \rightarrow h_{1}, h_{2} \rightarrow-h_{2}$ and $h_{1} \rightarrow-h_{1}, h_{2} \rightarrow h_{2}$, which get interchanged upon basis change $\phi_{2} \rightarrow i \phi_{2}$. Either can be used as the $C P$ symmetry of the model, making the specification of the $C P$ properties of $h_{1}$ and $h_{2}$ a basis dependent statement. Therefore, we denote the two neutral inert scalar masses as $M_{h_{1}}<M_{h_{2}}$, without specifying which is scalar and pseudoscalar. The masses of the physical scalars are

$$
\begin{aligned}
M_{H}^{2} & =2 \lambda_{1} v^{2}=2 m_{1}^{2}, \quad M_{h^{+}}^{2}=\frac{1}{2} \lambda_{3} v^{2}-m_{2}^{2}, \\
M_{h_{1}}^{2} & =\frac{1}{2}\left(\lambda_{3}+\lambda_{4}-\left|\lambda_{5}\right|\right) v^{2}-m_{2}^{2}, \\
M_{h_{2}}^{2} & =\frac{1}{2}\left(\lambda_{3}+\lambda_{4}+\left|\lambda_{5}\right|\right) v^{2}-m_{2}^{2}>M_{h_{1}}^{2} .
\end{aligned}
$$

The mass differences, written as

$M_{h_{2}}^{2}-M_{h_{1}}^{2}=\left|\lambda_{5}\right| v^{2}, \quad M_{h^{+}}^{2}-M_{h_{1}}^{2}=-\left(\lambda_{4}-\left|\lambda_{5}\right|\right) v^{2} / 2$, 


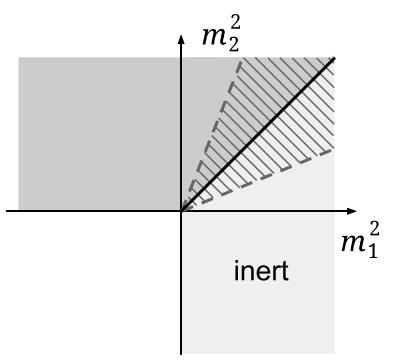

(a) $R>1$

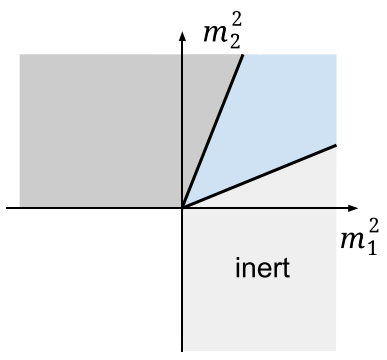

(b) $0<R<1$

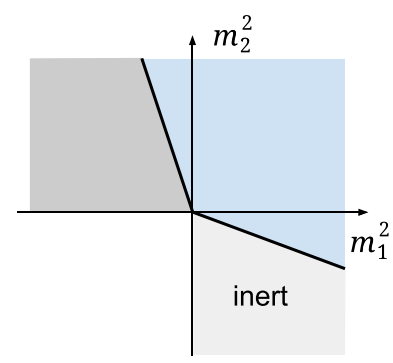

(c) $-1<R<0$

FIG. 1. Restrictions on the $\left(m_{1}^{2}, m_{2}^{2}\right)$ plane coming from the requirement that the inert vacuum is the deepest minimum of the potential. The three cases correspond to (a) $R>1$, (b) $0<R<1$, (c) $-1<R<0$. Light and dark grey correspond to models with an inert $v_{1}=v$, $v_{2}=0$ and a pseudoinert $v_{1}=0, v_{2}=v$ vacuum, respectively, while the blue region in between corresponds to the mixed vacuum, when both $v_{1}$ and $v_{2}$ are nonzero. The dashed region in the left plot indicates coexistence of the inert and pseudoinert minima at different depths.

highlight the role of the parameters $\lambda_{4}$ and $\lambda_{5}$ and are consistent with (5). It should also be stressed that the parameters $\lambda_{1}$ and $m_{1}^{2}$ correspond to the Higgs potential in the SM, and can thus be fixed by the values of the VEV and Higgs mass.

One also notices that the sign of $\lambda_{5}$ is phenomenologically irrelevant: flipping the sign of $\lambda_{5}$ would only lead to swapping the $C P$ parities of the inert neutral scalars, which are unobservable anyway. In order to eliminate double counting, we make the standard choice of $\lambda_{5}<0$ and introduce the shorthand notation $\lambda_{345}=\lambda_{3}+\lambda_{4}+\lambda_{5}$. The latter parameter plays an important phenomenological role, as it governs the Higgs-DM interaction vertex $H h_{1} h_{1}$. For future convenience, we also introduce the shorthand notation

$\tilde{\lambda}_{345} \equiv \lambda_{3}+\lambda_{4}-\lambda_{5}=\lambda_{345}+2\left|\lambda_{5}\right|=\lambda_{345}+\frac{2\left(M_{h_{2}}^{2}-M_{h_{1}}^{2}\right)}{v^{2}}$,

which is not a new free parameter and is the combination which governs, in particular, the $H h_{2} h_{2}$ coupling as well as the quartic coupling of $h_{1}$ to the longitudinal $Z$ bosons $h_{1} h_{1} Z_{L} Z_{L}$.

With all these conventions, we describe the fivedimensional parameter space of $\mathrm{i} 2 \mathrm{HDM}$ with the following phenomenologically relevant variables:

$$
\begin{aligned}
& M_{h_{1}}, \quad M_{h_{2}}>M_{h_{1}}, \quad M_{h^{+}}>M_{h_{1}}, \\
& \lambda_{2}>0, \quad \lambda_{345}>-2 \sqrt{\lambda_{1} \lambda_{2}} .
\end{aligned}
$$

Another set of theoretical constraints comes from the symmetry breaking patterns in i2HDM [24] and from the fact that the potential can have two minima at different depths. Following [44], we introduce $R=\lambda_{345} / 2 \sqrt{\lambda_{1} \lambda_{2}}$, which satisfies $R>-1$. Requiring that the inert vacuum corresponds to the global minimum leads to the following conditions on the parameters of the potential, apart from $m_{1}^{2}>0$ :

$$
\begin{aligned}
& m_{2}^{2}<\frac{\lambda_{345}}{2 \lambda_{1}} m_{1}^{2}=R \sqrt{\frac{\lambda_{2}}{\lambda_{1}}} m_{1}^{2}, \quad \text { if }|R|<1, \\
& m_{2}^{2}<\sqrt{\frac{\lambda_{2}}{\lambda_{1}}} m_{1}^{2}, \quad \text { if } R>1 .
\end{aligned}
$$

In Fig. 1 we visualize these restrictions on the $\left(m_{1}^{2}, m_{2}^{2}\right)$ plane for the three choices of $R$. The inert, $v_{1}=v, v_{2}=0$, and pseudoinert, $v_{1}=0, v_{2}=v$, vacua can coexist only when $R>1$, which is shown by the dashed region in Fig. 1(a). For $R>1$, the second line in Eq. (10) is a stronger condition than the first line, and it guarantees that the inert minimum is the deepest one. This condition is shown in Fig. 1(a) by the solid black line.

Rewriting conditions (10) for the physical parameters we get the constraint on the Higgs potential in the following compact final form:

$$
\text { the trivial one, } M_{h_{1}}^{2}>0 \text { for }|R|<1 \text {, }
$$

and

$$
\begin{aligned}
M_{h_{1}}^{2} & >\left(\lambda_{345} / 2 \sqrt{\lambda_{1} \lambda_{2}}-1\right) \sqrt{\lambda_{1} \lambda_{2}} v^{2} \\
& =(R-1) \sqrt{\lambda_{1} \lambda_{2}} v^{2} \text { for } R>1,
\end{aligned}
$$

where $\lambda_{1} \approx 0.129$ is fixed as in the Standard Model by the Higgs mass (6). The latter condition places an upper bound on $\lambda_{345}$ for a given DM mass $M_{h_{1}}$.

\section{B. Model implementation}

We have implemented the i2HDM into the CALCHEP package [65] with the help of the LANHEP program $[66,67]$ for automatic Feynman rules derivation. The effective $H g g$ and $H \gamma \gamma$ vertices were included, and the model was 
cross-checked in two different gauges to ensure a correct, gauge invariant implementation. It is publicly available at the High Energy Physics Model Data-Base (HEPMDB) [68] at http://hepmdb.soton.ac.uk/hepmdb:0715.0187 together with the LANHEP source of the model. The model is implemented in terms of the five independent parameters defined in Eq. (9), consisting of three physical masses and two couplings. We found this choice the most convenient for exploration of i2HDM phenomenology and constraints of its parameter space. We should stress that the $M_{h_{1}}$ and $M_{h_{2}}$ parameters conveniently define the mass order of the two neutral inert states independently of their $C P$ properties. This choice is especially convenient and relevant for collider phenomenology since, as we discussed above, one cannot assign (or determine) the $C P$ parity of each neutral inert scalar.

To explore the phenomenology of the i2HDM we need to consider other constraints on its parameter space in addition to those coming from vacuum stability which we discussed above.

\section{Constraints from perturbativity and unitarity}

The first requirement we impose on the quartic couplings in (2) is that their values are such that perturbative calculations can be trusted in the model. The most effective way is to impose perturbative unitarity on all the scattering processes involving the scalars. Following [51], we impose this condition on the full scattering matrix, which leads to the following bounds on combinations of couplings $e_{i}$ :

$$
\left|e_{i}\right| \leq 8 \pi
$$

where $e_{1,2}=\lambda_{3} \pm \lambda_{4}, e_{3,4}=\lambda_{3} \pm \lambda_{5}, e_{5,6}=\lambda_{3}+2 \lambda_{4} \pm 3 \lambda_{5}$, $e_{7,8}=-\lambda_{1}-\lambda_{2} \pm \sqrt{\left(\lambda_{1}-\lambda_{2}\right)^{2}+\lambda_{4}^{2}}, \quad e_{9,10}=-3 \lambda_{1}-$ $3 \lambda_{2} \pm \sqrt{9\left(\lambda_{1}-\lambda_{2}\right)^{2}+\left(2 \lambda_{3}+\lambda_{4}\right)^{2}}, \quad e_{11,12}=-\lambda_{1}-\lambda_{2} \pm$ $\sqrt{\left(\lambda_{1}-\lambda_{2}\right)^{2}+\lambda_{5}^{2}}$. The parameter $\lambda_{1}$ is fixed by SMHiggs mass and the vacuum expectation value. One can verify that the constraints given by Eq. (13) imply that all quartic couplings in (2) are bound to be smaller than $8 \pi$, thus within the perturbative regime. The perturbativity constraints can also be used to find upper bounds on the two input couplings we defined in the previous section, i.e. $\lambda_{2}$ and $\lambda_{345}$. From $e_{10}$ one finds

$$
\lambda_{2}<\lambda_{2}^{\max }<4 \pi / 3
$$

where $\lambda_{2}^{\max }$ is a function of model parameters, while from $e_{5}=3 \lambda_{345}-\left(2 \lambda_{3}+\lambda_{4}\right)$, combined with $e_{10}$ in the limit $\lambda_{2}=0$, we obtain an upper bound for $\lambda_{345}$,

$$
\begin{aligned}
-1.47 & \simeq-2 \sqrt{\lambda_{1}(4 \pi / 3)}<-2 \sqrt{\lambda_{1} \lambda_{2}^{\max }}<\lambda_{345} \\
& \lesssim \frac{2}{3} \times 8 \pi-\lambda_{1},
\end{aligned}
$$

where we expanded at leading order in the small coupling $\lambda_{1}$, and the lower bound comes from the stability of the potential. This limit, derived from the constraints on $e_{5}$ and $e_{10}$ is not actually the most stringent one: in the limit of $\lambda_{2} \rightarrow 0$ we have found that the biggest value for $\lambda_{345}$ is realized in the $\left|\lambda_{4,5}\right| \rightarrow 0$ limit when $\lambda_{3} \simeq 4 \pi$ and, respectively, $\lambda_{345} \simeq 4 \pi$. After expansion in the small coupling $\lambda_{1}$, the upper limit on $\lambda_{345}$ in the small $\lambda_{2}$ limit reads as

$$
\lambda_{345} \lesssim 4 \pi-\frac{3}{2} \lambda_{1}
$$

while for finite $\lambda_{2}$ the limit can be found numerically. We would like to note that the limit from perturbative unitarity and perturbativity given by Eqs. (13)-(16) we are using in our study is consistent with that implemented in the 2HDMC code [69].

One should also stress that the vacuum stability condition given by Eq. (12) sets an important constraint on the maximum value of $\lambda_{345}$ in the small $M_{h_{1}}$ region (which is the region of our special interest because of the collider phenomenology constraints as we discuss below). This can be seen from Eq. (12) which can be written as

$$
\lambda_{345}<2\left(\frac{M_{h_{1}}^{2}}{v^{2}}+\sqrt{\lambda_{1} \lambda_{2}^{\max }}\right) .
$$

In Fig. 2, we present viable parameter space in the $\left(\lambda_{345}, \lambda_{2}\right)$ plane after constraints from Eq. (13) as well as constraints from the scalar potential given by Eqs. (11), (12), (17). To produce this plot we have performed the wide random scan to cover the full five-dimensional parameter space of the model, with the following chosen range for the model parameters:

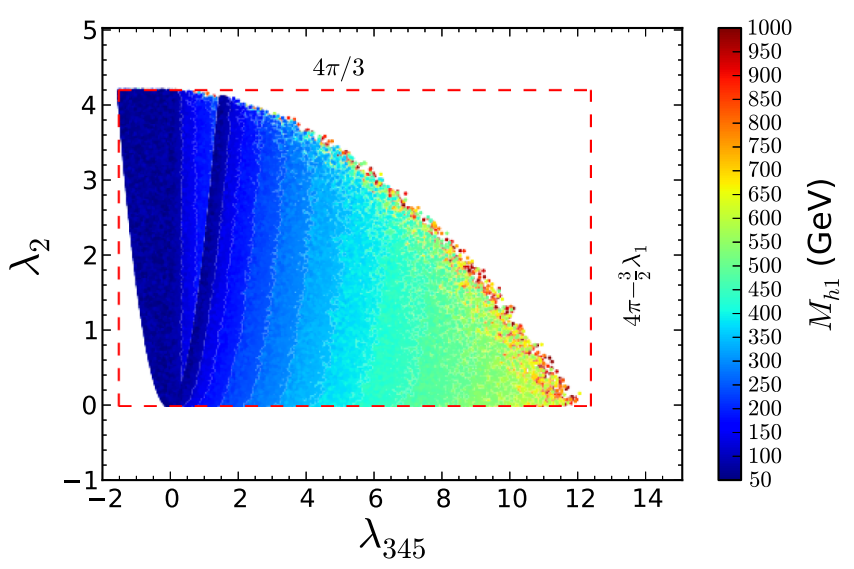

FIG. 2. The part of the $\left(\lambda_{345}, \lambda_{2}\right)$ parameter space allowed by the unitarity, perturbativity, and scalar potential constraints. 


$$
\begin{aligned}
10 \mathrm{GeV} & <M_{h 1, h 2, h^{+}}<1000 \mathrm{GeV} \\
0 & <\lambda_{2}<\frac{4 \pi}{3} \\
-1.47 & <\lambda_{345}<4 \pi .
\end{aligned}
$$

The color map in Fig. 2 presents the values for the third essential parameter, the DM candidate mass $M_{h_{1}}$, with points of smaller values of $M_{h_{1}}$ on the top of points with larger $M_{h_{1}}$ values. From Fig. 2 one can observe a nontrivial shape of the allowed parameter space in the $\left(\lambda_{345}, \lambda_{2}\right)$ plane defined by the constraints mentioned above. In particular, for small $M_{h_{1}}$ values, the upper limit on $\lambda_{345}$ comes from Eq. (17) which restricts $H h_{1} h_{1}$ coupling $\lambda_{345}$ to be not very large. The value of $\lambda_{2}^{\max }$ entering there can be found in general only numerically.

\section{Constraints from LEP and electroweak precision data}

Very strong constraints on the i2HDM arise from precision data and searches from LEP experiments. First of all, the model should respect the precise measurements of the $W$ and $Z$ widths which lead to the following lower limit on the odd scalar masses:

$$
\begin{aligned}
M_{h_{1}}+M_{h^{+}}>M_{W^{+}}, & M_{h_{2}}+M_{h^{+}}>M_{W^{+}}, \\
M_{h_{1}}+M_{h_{2}}>M_{Z}, & 2 M_{h^{+}}>M_{Z},
\end{aligned}
$$

to make sure that $\Gamma\left(W^{+} \rightarrow h_{1} h^{+}, h_{2} h^{+}\right)$and $\Gamma\left(Z \rightarrow h_{1} h_{2}, h^{+} h^{-}\right)$decay channels are kinematically forbidden.

While studying the phenomenology of the i2HDM, we should also make sure that Electroweak Precision Test (EWPT) data are respected. As we know, EWPT can be expressed in terms of three measurable quantities, called $S$, $T$, and $U$, that parametrize contributions from beyond standard model physics to electroweak radiative corrections [70]. The contribution to the $S$ and $T$ parameters [26] can be written as

$$
\begin{aligned}
S= & \frac{1}{72 \pi} \frac{1}{\left(x_{2}^{2}-x_{1}^{2}\right)^{3}}\left[x_{2}^{6} f_{a}\left(x_{2}\right)-x_{1}^{6} f_{a}\left(x_{1}\right)\right. \\
& +9 x_{2}^{2} x_{1}^{2}\left(x_{2}^{2} f_{b}\left(x_{2}\right)-x_{1}^{2} f_{b}\left(x_{1}\right)\right]
\end{aligned}
$$

where $\quad x_{1}=M_{h_{1}} / M_{h^{+}}, \quad x_{2}=M_{h_{2}} / M_{h^{+}}, \quad f_{a}(x)=$ $-5+12 \log (x), f_{b}(x)=3-4 \log (x)$, and

$$
\begin{aligned}
T= & \frac{1}{32 \pi^{2} \alpha v^{2}}\left[f_{c}\left(M_{h^{+}}^{2}, M_{h_{2}}^{2}\right)+f_{c}\left(M_{h^{+}}^{2}, M_{h_{1}}^{2}\right)\right. \\
& \left.-f_{c}\left(M_{h_{2}}^{2}, M_{h_{1}}^{2}\right)\right]
\end{aligned}
$$

where the function $f_{c}(x, y)$ is defined by

$$
f_{c}(x, y)=\left\{\begin{array}{ll}
\frac{x+y}{2}-\frac{x y}{x-y} \log \left(\frac{x}{y}\right), & x \neq y \\
0, & x=y
\end{array} .\right.
$$

We have written the contributions to $S$ and $T$ in a form which demonstrates explicitly their symmetry with respect to swapping $h_{1} \leftrightarrow h_{2}$, pointing again to the fact that one cannot distinguish their $C P$ properties. With $U$ fixed to be zero, the central values of $S$ and $T$, assuming a SM Higgs boson mass of $m_{h}=125 \mathrm{GeV}$, are given by [71]

$$
S=0.06 \pm 0.09, \quad T=0.1 \pm 0.07
$$

with correlation coefficient +0.91 . The effect of the constraints on $S$ and $T$ is presented in Fig. 3, where panels 3(a) and 3(b) present the color map of the $S$ and $T$ parameters, respectively, in the $\left(M_{h^{+}}, M_{h_{2}}\right)$ plane. One can see that the $T$ variable is more sensitive than $S$ to this mass split; thus only modest splits are allowed by EWPT data. Finally, Fig. 3(c) presents the color map of the $M_{h^{+}}-M_{h_{2}}$ split in the $(S, T)$ plane together with the $65 \%$ and $95 \%$ exclusion contours, based on a $\chi^{2}$ with 2 degrees of freedom. One can see that EWPT data prefer a modest positive $M_{h^{+}}-M_{h_{2}}$ mass split below about $100 \mathrm{GeV}$, which is mainly defined by the $T$ parameter, while the role and the respective range of variation of $S$ is milder. One should stress that it is crucial to take into account the correlation between $S$ and $T$ and combine limits from these two parameters. This combination gives a much stronger limit on the parameter space, in particular on the $M_{h^{+}}-M_{h_{2}}$ mass split, while a much larger splitting would naively be allowed by looking at the $S$ and $T$ values separately. This can be seen from Figs. 3(a) and 3(b), respectively.

We also excluded the region defined by the intersection of the conditions below:

$$
\begin{aligned}
& M_{h_{1}}<80 \mathrm{GeV}, \quad M_{h_{2}}<100 \mathrm{GeV}, \\
& M_{h_{2}}-M_{h_{1}}>8 \mathrm{GeV} .
\end{aligned}
$$

This region is excluded by the LEP data since it would lead to a visible dijet or dilepton signal as demonstrated in [72] where a reinterpretation in the 2 2HDM of a LEP-II limit of the second neutralino production in the Minimal Supersymmetric Standard Model (MSSM) was presented. A more detailed analysis of this specific region of the parameter space-low $M_{h_{1}}$ and $M_{h_{2}}$ with large enough mass gap-was studied recently [73]. One should also mention that $e^{+} e^{-} \rightarrow h^{+} h^{-}$production at LEP2 sets

$$
M_{h^{+}}>70 \mathrm{GeV}
$$

as found in [74] as a result of the reinterpretation of LEP-II limits on charginos. 


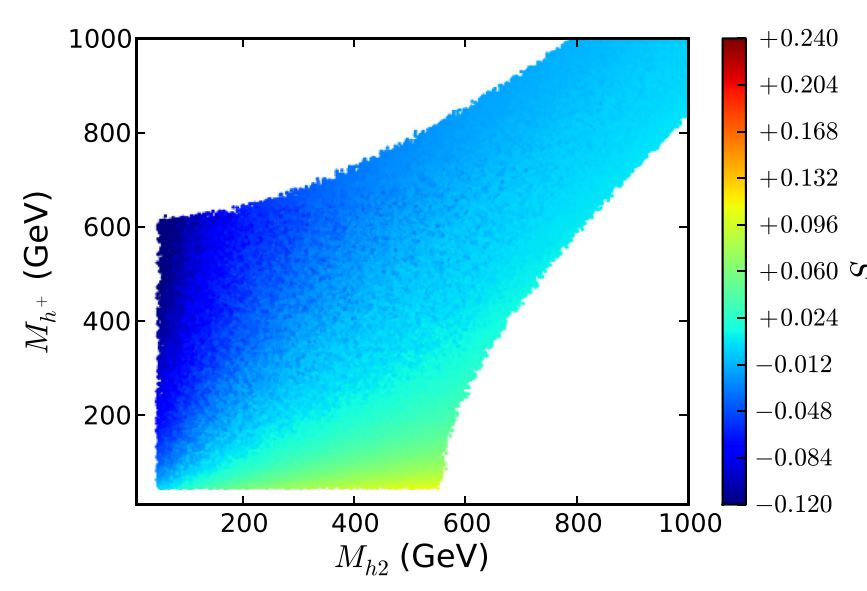

(a)

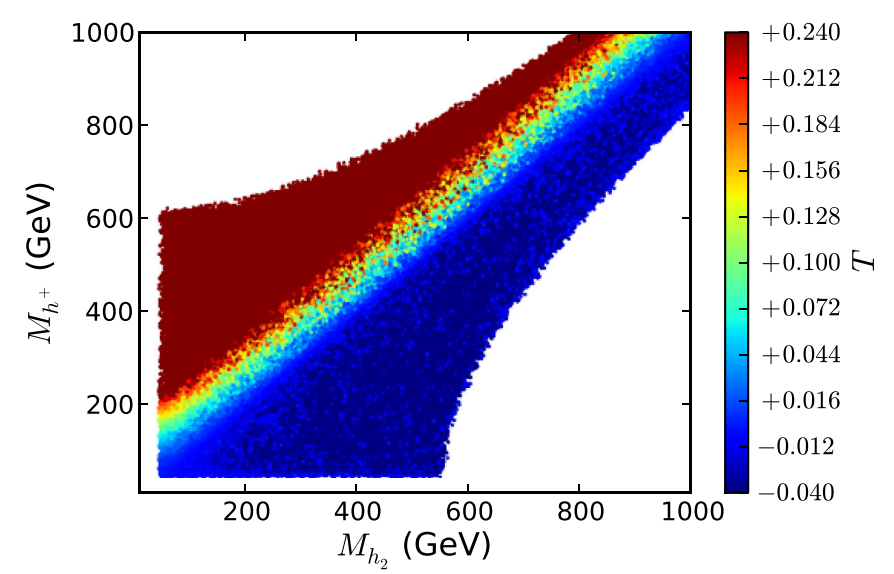

(b)

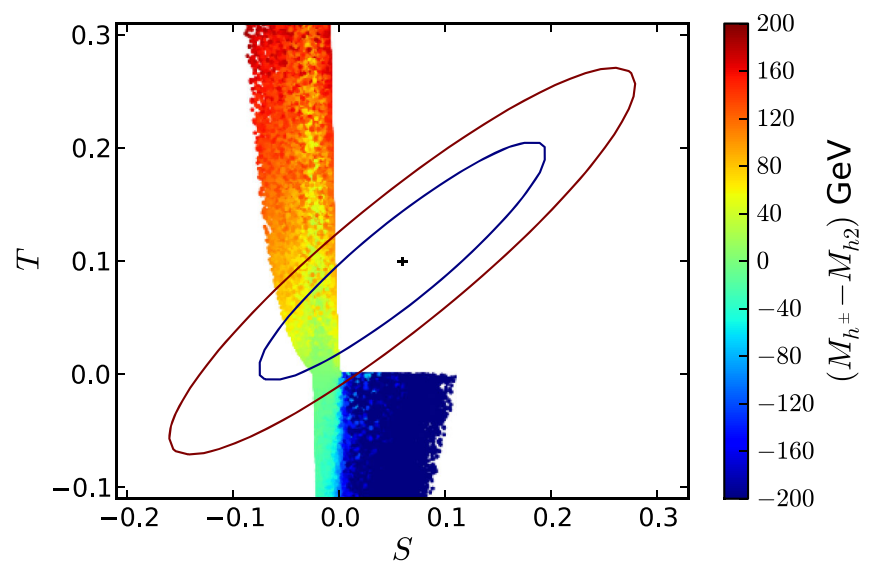

(c)

FIG. 3. Effect of the $S$ and $T$ constraints on the $M_{h^{+}}-M_{h_{2}}$ mass difference: (a) and (b) show the color map of the $S$ and $T$ parameters, respectively, in the $\left(M_{h^{+}}, M_{h_{2}}\right)$ plane; (c) shows the color map of the $M_{h^{+}}-M_{h_{2}}$ split in the $(S, T)$ plane together with the $65 \%$ and $95 \%$ exclusion contours based on the $\chi^{2}(S, T)$ characterization for 2 degrees of freedom.

\section{E. Constraints from LHC Higgs data}

The LHC Higgs data further restrict the i2HDM parameters space in the form of constraints on the couplings of the SM-like Higgs boson. A collection of combined fits from the Run I data, for both ATLAS and CMS, can be found in [75]. In the i2HDM, the leading effect is encoded in two observables: the decays of the Higgs into two dark matter scalars, $H \rightarrow h_{1} h_{1}$, which is kinematically open when $M_{h_{1}}<M_{H} / 2$; and the contribution of the charged Higgs loops to the $H \rightarrow \gamma \gamma$ decay. In principle, we would need to do a two-parameter fit of the available Higgs data. None of the fits presented in [75] can therefore be directly applied in our case.

A simpler possibility is, instead, to consider the best possible bound from the available fits on the two parameters. We follow this simpler procedure, confident that it will lead to a somewhat more conservative estimation of the bounds. For the invisible Higgs branching ratio, we consider the bound coming from the dedicated ATLAS search [76]

$$
\operatorname{Br}(H \rightarrow \text { invisible })<28 \%
$$

at the $95 \%$ C.L., which is comparable with a $36 \%$ limit from the combined CMS analysis [77]. ${ }^{2}$

For the second observable, the diphoton decay rate, we consider the result from the combined fit on the signal strength in the diphoton channel [75]:

$$
\frac{\operatorname{Br}^{\mathrm{BSM}}(H \rightarrow \gamma \gamma)}{\mathrm{Br}^{\mathrm{SM}}(H \rightarrow \gamma \gamma)}=\mu^{\gamma \gamma}=1.14_{-0.36}^{+0.38}
$$

where we doubled the $1 \sigma$ errors given in [75] to obtain the $\mu^{\gamma \gamma}$ range at the $95 \%$ C.L. A sufficiently light charged

\footnotetext{
${ }^{2}$ One could also limit $\operatorname{Br}(H \rightarrow$ invisible $)$ using $\operatorname{Br}(H \rightarrow$ $\mathrm{BSM})<34 \%$ at $95 \%$ C.L. exclusion from Run 1 ATLASCMS Higgs data analysis [75]. However, here we use the $\operatorname{Br}(H \rightarrow$ invisible $)<28 \%$ limit from a dedicated ATLAS search as it is less model dependent.
} 
Higgs with sufficiently large $\lambda_{3}$ coupling to the SM Higgs boson, which would bring the $H \rightarrow \gamma \gamma$ decay beyond the quoted limit, is excluded.

It should be noted that we would expect a proper twoparameter fit to lead to stronger constraints than the ones we use; however, the qualitative impact of the constraints should be unchanged. For example, the partial decay width of the Higgs into DM which is defined by

$$
\Gamma\left(H \rightarrow h_{1} h_{1}\right)=\frac{1}{8 \pi} \frac{\lambda_{345}^{2} M_{W}^{2}}{g_{W}^{2} M_{H}} \sqrt{1-4 \frac{M_{h_{1}}^{2}}{M_{H}^{2}}},
$$

where $g_{W}$ is the weak coupling constant, provides the following bound on $\lambda_{345}$ :

$$
\left|\lambda_{345}\right|<\left(\frac{8 \pi g_{W}^{2} \Gamma_{\mathrm{SM}} M_{H}}{M_{W}^{2}\left(\frac{1}{\mathrm{Br}_{\text {invis }}^{\max }}-1\right) \sqrt{1-4 \frac{M_{h_{1}}^{2}}{M_{H}^{2}}}}\right)^{1 / 2},
$$

where $\mathrm{Br}_{\text {invis }}^{\max }=0.28$ is the current bound on the maximal value of branching ratio of the Higgs boson decay into invisible mode. The above limit on $\lambda_{345}$ is $M_{h_{1}}$ dependent: for $M_{h_{1}} / M_{H} \ll 1$ it is about 0.019 , while for $M_{h_{1}}$ closer to the threshold, e.g. $60 \mathrm{GeV}$, the limit on $\lambda_{345}$ increases almost by a factor of 2 and reaches a value of 0.036 . In addition, we have included the limit from $H \rightarrow h_{2} h_{2}$ when $h_{2}$ is close in mass to $h_{1}$, which can be trivally done, taking into account that $H h_{2} h_{2}$ coupling is equal to $\tilde{\lambda}_{345}$ in Eq. (8). We discuss these limits in more details below, together with the DM constraints.

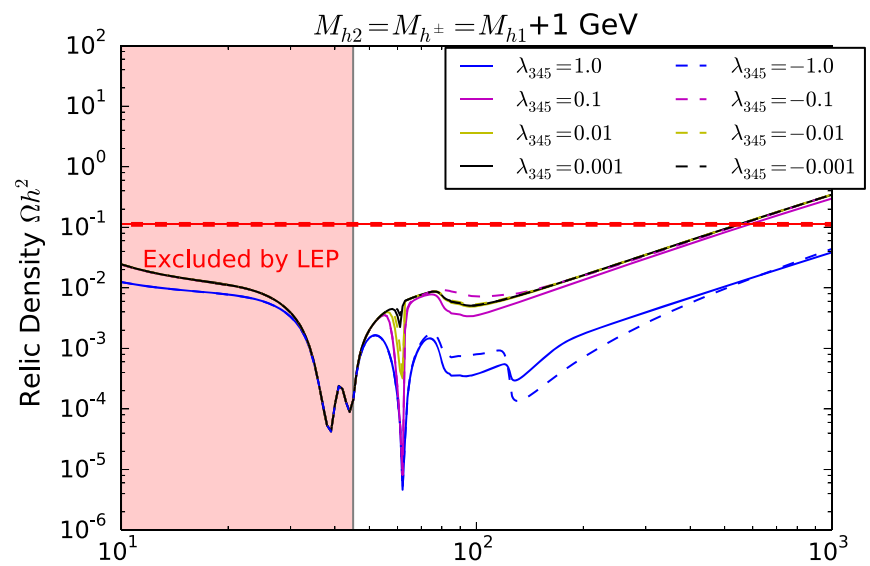

(a) $\mathrm{Mh}_{1}(\mathrm{GeV})$

\section{F. Dark matter relic density and direct/indirect detection}

The results from PLANCK [78,79] (see also WMAP [80]) have further decreased the error on the already quite precise measurement of the dark matter relic density, $\Omega_{\mathrm{DM}} h^{2}$ :

$$
\Omega_{\mathrm{DM}}^{\mathrm{Planck}} h^{2}=0.1184 \pm 0.0012 .
$$

In the i2HDM model, the lightest inert scalar $h_{1}$ is stable and contributes to this relic density. In our study we take the upper limit on $\Omega_{\mathrm{DM}} h^{2}$ as the hard one, excluding the parameter space points which lead to DM overabundance. However, we do not exclude the i2HDM parameter space regions where $h_{1}$ is underabundant, allowing for other sources of DM coming from an additional new physics sector.

We have evaluated $\Omega_{\mathrm{DM}} h^{2}$ with the MICROMEGAs 2.4.1 package [81-83] since it directly reads the model files in CALCHEP format. In our analysis we have assumed $10 \%$ theoretical uncertainty on the DM relic density prediction since it is based on the tree-level calculation. This uncertainty is the dominant one in comparison to about $1 \%$ uncertainty on DM fit from the latest PLANCK results given above and relax the DM relic density limit to the following one at $95 \%$ C.L.:

$$
\begin{aligned}
\Omega_{\mathrm{DM}}^{\operatorname{limit}} h^{2} & =0.1184 \pm 2 \times 0.1184 / 10 \\
& \simeq 0.118 \pm 2 \times 0.012
\end{aligned}
$$

which we will refer here still as "PLANCK limit."

Figure 4(a) shows the relic density in the case of quasidegenerate $h_{1}, h_{2}$, and $h^{+}$masses, $M_{h_{2}}=M_{h^{+}}=$ $M_{h_{1}}+\Delta M=M_{h_{1}}+1 \mathrm{GeV}$. This case is qualitatively

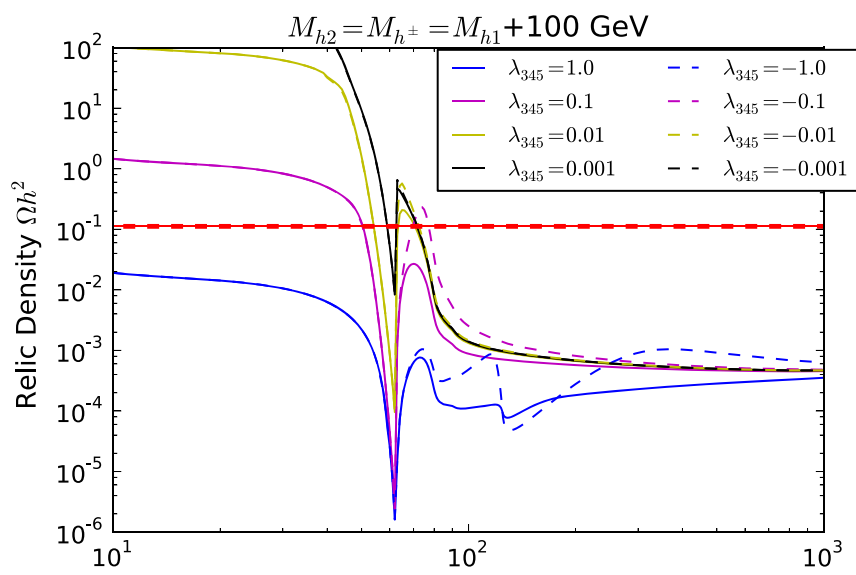

(b) $\mathrm{Mh}_{1}(\mathrm{GeV})$

FIG. 4. The relic density, $\Omega_{\mathrm{DM}} h^{2}$, as a function of $M_{h_{1}}$ for various $\lambda_{345}$ parameters. The red-shaded region in the left frame is excluded by the LEP data, since in this region $W$ and $Z$ bosons would decay to the light inert scalars. The horizontal red line corresponds to the relic density upper limit given by Eq. (30). 
different from the case with a non-negligible mass splitting as illustrated in Fig. 4(b), where we chose $M_{h_{2}}=M_{h^{+}}=$ $M_{h_{1}}+\Delta M=M_{h_{1}}+100 \mathrm{GeV}$. One should also note that scenarios with positive or negative $\lambda_{345}$ values of the same magnitude are qualitatively similar, except for the effect of interference (see dashed versus solid curves in Fig. 4). One can observe the following effects and features of the model in Fig. 4:

(i) The red-shaded region in Fig. 4(a) is excluded by the LEP data, since in this region $W$ and $Z$ bosons would decay to the light inert scalars. Respectively, the effect of the resonant coannihilation, $h_{1} h_{2} \rightarrow Z$ and $h_{1} h^{+} \rightarrow W^{+}$, can be seen in this region in the first two dips for $M_{h_{1}} \sim 40$ and $45 \mathrm{GeV}$. These processes are governed by the gauge coupling constant and are independent of $\lambda_{345}$.

(ii) In the case of larger $M_{h_{2}}-M_{h_{1}}$ mass split [Fig. 4(b)], this effect disappears since $M_{h_{1}}+M_{h_{2}}>M_{Z}$ and $M_{h_{1}}+M_{h^{+}}>M_{W}$.

(iii) The sharpest dip in the $\Omega_{\mathrm{DM}} h^{2}$ dependence of $M_{h_{1}}$ is at $65 \mathrm{GeV}$ and corresponds to the DM annihilation through the Higgs boson $h_{1} h_{1} \rightarrow H$. It is present in both cases.

(iv) At higher masses, we observe a wider and more shallow dip at around 80-90 GeV from $h_{1} h_{1} \rightarrow$ $W^{+} W^{-}$and $h_{1} h_{1} \rightarrow Z Z$ channels which are merged together.

(v) Finally, the last dip around $125 \mathrm{GeV}$ corresponds to the reduction of the DM relic density due to the opening of the $h_{1} h_{1} \rightarrow H H$ annihilation channel. This dip takes place only for large values of $\lambda_{345}$, which provide a high enough rate for the $h_{1} h_{1} \rightarrow$ $H H$ process via the $s$-channel Higgs boson.

(vi) The pattern of these last three dips is the same for the larger mass split scenario presented in Fig. 4(b). In both scenarios, the interference effect is sensitive to the sign of $\lambda_{345}$ and appears in this region as a result of the positive or negative interference of the $s$ channel Higgs boson exchange diagram and the rest of annihilation diagrams.

(vii) One can also observe qualitative differences in the asymptotic behavior of the DM relic density for small and large $M_{h_{1}}$ values for different $\Delta M$. In the $\Delta M=1 \mathrm{GeV}$ case with $M_{h_{1}}<65 \mathrm{GeV}$, the effective coannihilation of the inert scalars keeps the DM density always below the PLANCK limit. For $\Delta M=100 \mathrm{GeV}$, DM coannihilation is suppressed and the relic density is equal or below the experimental limit only for large values of $\lambda_{345}$ $\left(\lambda_{345} \gtrsim 0.3\right)$ which are excluded by LHC limits on the invisible Higgs decay; see Eq. (28).

(viii) For $M_{h_{1}}$ well above $65 \mathrm{GeV}$, coannihilation effects become less important in comparison with $h_{1} h_{1}$ annihilation into vector bosons, which opens in this region. For this annihilation process the quartic couplings of DM with longitudinal vector bosons $h_{1} h_{1} V_{L} V_{L}$ play an important role. For $h_{1} h_{1} Z_{L} Z_{L}$, it is equal to $\tilde{\lambda}_{345}$ defined in (8), while for $h_{1} h_{1} W_{L} W_{L}$ it is given by $\lambda_{3}=\lambda_{345}+2\left(M_{h^{+}}^{2}-M_{h_{1}}^{2}\right) / v^{2}$. For small mass splittings $\Delta M_{c}=M_{h^{+}}-M_{h_{1}}$ and $\Delta M_{2}=M_{h_{2}}-M_{h_{1}}$, the correspondingly small values of the $h_{1} h_{1} V_{L} V_{L}$ quartic couplings generate a low $h_{1} h_{1}$ annihilation cross section $\langle\sigma v\rangle$, which decreases with growing $M_{h_{1}}$. Eventually this leads to a comparatively high value of $\Omega_{\mathrm{DM}} h^{2}$ (which increases with $M_{h_{1}}$ both due to the decrease of $\langle\sigma v\rangle$ as well as the increase of the DM mass) which reaches the PLANCK limit for large enough $M_{h_{1}}$ as one can see from Fig. 4(a). On the contrary, for large $\Delta M_{c}$ and/or $\Delta M_{2}$, the mass splittings generate a high rate for $h_{1} h_{1}$ annihilation into vector bosons, which rises with growing $M_{h_{1}}$. This generates a DM density below the experimental limit even for large values of $M_{h_{1}}$. In this scenario the potential increase of $\Omega_{\mathrm{DM}} h^{2}$ due the large DM mass is compensated by the respective increase of $\langle\sigma v\rangle$ and leads to an approximately flat $\Omega_{\mathrm{DM}} h^{2}$ versus $M_{h_{1}}$ in the 100$1000 \mathrm{GeV}$ range. This makes the asymptotic behavior of the DM density versus $M_{h_{1}}$ qualitatively different for $\Delta M=100 \mathrm{GeV}$ as compared to $\Delta M=1 \mathrm{GeV}$; see Fig. 4(b). These two scenarios with the large and small $\Delta M_{c}, \Delta M_{2}$ mass splittings qualitatively cover the whole parameter space of the i2HDM.

We have also checked whether the i2HDM parameter space is consistent with the limits from DM DD experiments. We have evaluated the spin-independent cross section of DM scattering off the proton, $\sigma_{\mathrm{SI}}$, also using the MICROMEGAs package. In Fig. 5 limits from LUX100 are shown by the shaded green area where the left and right frames illustrate the small and large $\Delta M$ scenarios as in Fig. 4. To present the results in Fig. 5, we use the rescaled DD cross section, $\hat{\sigma}_{\mathrm{SI}}=R_{\Omega} \times \sigma_{\mathrm{SI}}$, where the scaling factor $R_{\Omega}=\Omega_{\mathrm{DM}} / \Omega_{\mathrm{DM}}^{\text {Planck }}$ takes into account the case of $h_{1}$ representing only a part of the total DM budget, thus allowing for a convenient comparison of the model prediction with the limits from LUX [84].

The flat asymptotic of $\hat{\sigma}_{\mathrm{SI}}$ in Fig. 5(a) for high $M_{h_{1}}$ means that the decrease of the proton-DM scattering cross section $\sigma_{\mathrm{SI}}$ with increasing $M_{h_{1}}$ is compensated by the growth of the relic density which one can observe in Fig. 4(a). In Fig. 5(b), on the other hand, $\hat{\sigma}_{\mathrm{SI}}$ drops with large and increasing values of $M_{h_{1}}$. This can be understood by observing from Fig. 4(b) that in this region $R_{\Omega}=$ $\Omega_{\mathrm{DM}} / \Omega_{\mathrm{DM}}^{\text {Planck }} \simeq$ const, and therefore the asymptotic behavior of $\hat{\sigma}_{\mathrm{SI}}$ is the same as for $\sigma_{\mathrm{SI}}$; that is, it goes down as $M_{h_{1}}$ grows due to the reduced $\langle\sigma v\rangle$.

A related question is whether the model can be better probed by indirect detection (ID) experiments, i.e. the 


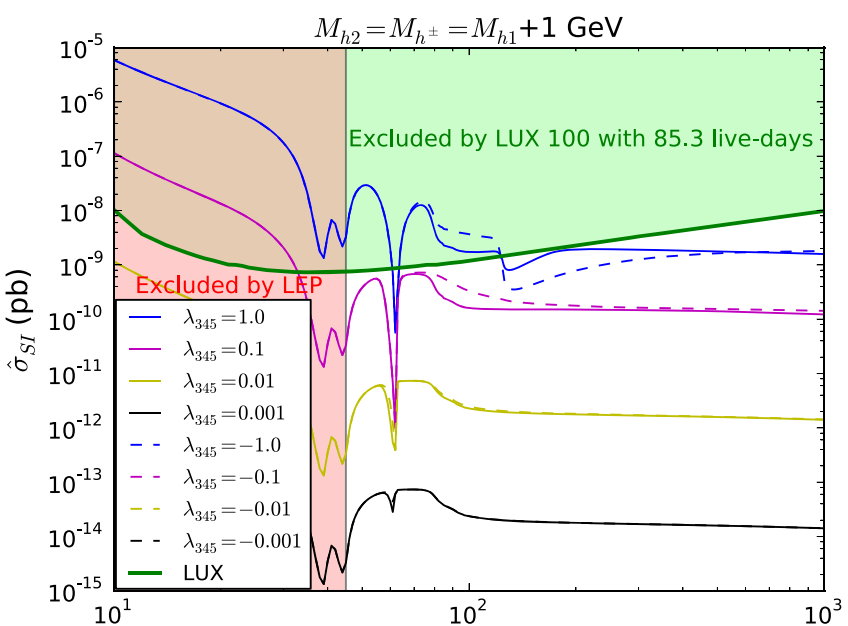

(a) $M_{h 1}(\mathrm{GeV})$

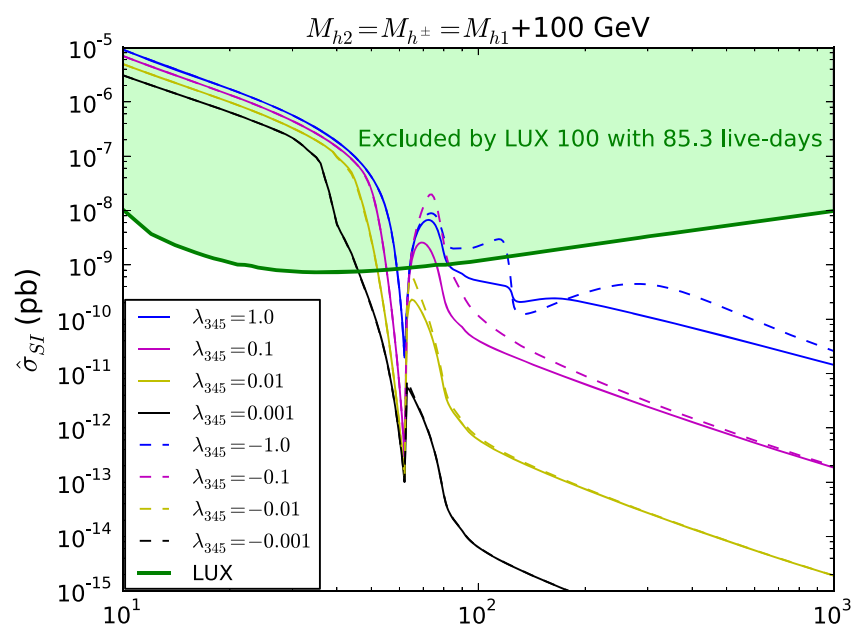

(b) $M_{h 1}(\mathrm{GeV})$

FIG. 5. Rescaled spin independent direct detection rates $\hat{\sigma}_{\mathrm{SI}}$ versus $M_{h_{1}}$ and the LUX100 constraint. The red-shaded region in the left frame is excluded by LEP data.

detection of energetic cosmic rays like $e^{+}, \gamma, p$, or $\bar{p}$, which may be created by the annihilation of $h_{1}$ pairs. We have checked that the strongest bounds on the i2HDM parameter space coming from such experiments are set by gamma ray telescopes: both the Fermi-LAT gamma-ray space telescope [85] as well as ground based telescopes. Fermi-LAT is sensitive to gamma rays particularly in the low mass range up to $\mathcal{O}(100 \mathrm{GeV})$, but the bounds are not competitive with those coming from DD. This conclusion is also confirmed by studies in Ref. [60]. Another recent work, Ref. [86], indirectly confirms that the present Fermi-LAT data do not place additional strong constraints on the i2HDM parameter space. The authors of that work looked at the so-called gamma-ray Galactic center excess [87] and asked if it can be explained via the DM annihilation in i2HDM. They indeed found a few possible regions, and estimate that one would need 15 years of Fermi-LAT data to conclusively test it. In our work, we stay conservative and do not interpret such signals as the DM evidence. We can only state that the regions selected in Ref. [86] as promising are, at best, not excluded by Fermi-LAT data. Since the other regions were not favored by the Galactic center excess in the Fermi-LAT data, their expected contributions to the ID signals are weaker. Finally, we stress that incorporating ID limits into our picture would bring novel uncertainties of purely astrophysical origin such as the poorly known DM distribution profile. Thus, they cannot easily be translated into a new constraint on the i2HDM parameter space.

\section{NUMERICAL SCAN OF THE PARAMETER SPACE}

\section{A. Results of the general scan}

To have a complete picture of the properties of i2HDM in the whole parameter space, we have performed a five-dimensional random scan of the model parameter space with about $10^{8}$ points, evaluating all relevant observables and limits mentioned above. The range for the model parameters of the scan was chosen according to Eq. (18).

When performing the scan, we took into account the constraints mentioned above in the following succession. First, we applied only theoretical constraints from vacuum stability, perturbativity, and unitarity; second, we applied the collider constraints (LEP, EWPT, LHC Higgs data); last, we placed the upper bound on the DM relic density at $\Omega_{\mathrm{DM}} h^{2} \leq 0.1184+2 \times 0.0012$ given by the PLANCK result plus 2 standard deviations, and took into account the negative results of the DM DD searches at LUX.

The salient features of the results of this scan, with all three groups of constraints applied successively, are presented in Fig. 6 on the $\left(M_{h_{1}}, \lambda_{345}\right)$ projection. The results are presented in the form of color maps, where the color encodes the value of the thermal relic density. The points with higher relic density are always on top of those with low density. To make the exposition as clear as possible, we decided to show here only this specific two-dimensional (2D) projection. The reader can find more information and insights in Appendix, which contains more projections and a more detailed description of the effects of each set of constraints. Our scan highlights the following features:

(i) The lower bound of $\lambda_{345}$ corresponds to the theoretical lower limit in Eq. (18). The upper bound on $\lambda_{345}$ depends on $M_{h_{1}}$ and comes from the vacuum stability condition given in Eq. (17). Taking into account the collider constraints, and in particular the invisible Higgs decay and the $H \gamma \gamma$ coupling strength, restricts $\lambda_{345}$ to $\left|\lambda_{345}\right| \leq 0.02$ at $M_{h_{1}}<M_{H} / 2$.

(ii) The LEP and LHC data also place constraints on the other inert scalars. Charged scalars lighter than $70 \mathrm{GeV}$ as well as $M_{h_{2}}<M_{Z} / 2$ are generically 

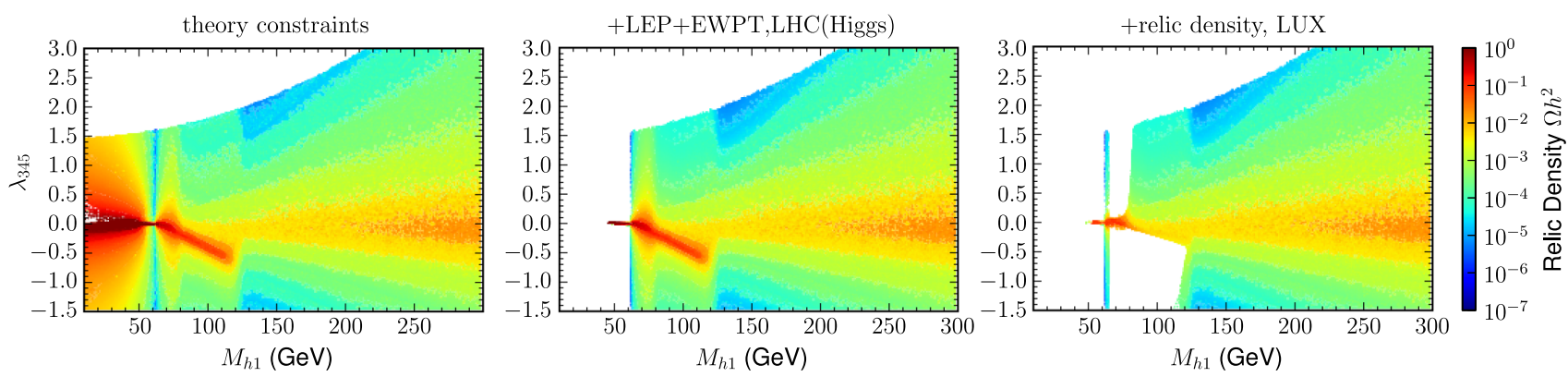

FIG. 6. Color maps of DM relic abundance projected on the plane $\left(M_{h_{1}}, \lambda_{345}\right)$. The three plots correspond to the surviving points after progressively imposing the three layers of constraints described in the text.

excluded. For $M_{h_{2}}$ above this value and below approximately $100 \mathrm{GeV}$, the only surviving region is a narrow strip with $M_{h_{2}}-M_{h_{1}}<8 \mathrm{GeV}$. The effect from these constraints can be seen in Fig. 7 which shows the points surviving all constraints in a projection in the masses, and more details can be found in Appendix.

(iii) The narrow strip at $M_{h_{1}}<M_{H} / 2$ surviving after collider data, which is seen in Fig. 6(b), is further cut off once the relic density constraint, in addition, is taken into account. Indeed, for such a small $M_{h_{1}}$ and $\lambda_{345}$ (and not too small $M_{h_{2}}$ to prevent $Z \rightarrow h_{1} h_{2}$ decays), there remains no mechanism for sufficiently active removal of DM in the early universe. The resulting DM relic density turns out too high and is ruled out. We already saw this feature in Fig. 4(b). Values of $M_{h_{1}}>45 \mathrm{GeV}$ are still allowed but they require a close $M_{h_{2}}$ for an efficient coannihilation in the early universe. This region is well visible as a protrusion in the $\left(M_{h_{1}}, M_{h_{2}}\right)$ in Fig. 7.

(iv) The masses $M_{h_{1}}>M_{H} / 2$ are not constrained by the relic density, but the DM DD results from LUX cut off a part of the parameter space. This is visible in the last plot in Fig. 6, for $M_{h_{1}} \lesssim M_{H}$ and with large and intermediate $\left|\lambda_{345}\right|$. In this region, the sizable $\left|\lambda_{345}\right|$ simultaneously keeps the relic density below the Planck upper bound and allows the scattering cross section to be enhanced due to the Higgs boson exchange. The interplay of moderately low relic density and a sizable cross section leads to a DD signal which could have been seen by LUX. For larger DM masses, the direct annihilation into $W W$, $\mathrm{ZZ}, \mathrm{HH}$ pairs opens up, and the relic density drops further, making LUX insensitive to this region.

(v) Finally, we remark that above $200 \mathrm{GeV}$, EWPT forces $M_{h_{2}}$ and $M_{h^{+}}$to stay rather close to each other, see again Appendix for more details.

In summary, after all constraints mentioned here and exposed in more detail in Appendix, we found that the parameter space with

$$
M_{h_{1}}, M_{h_{2}}<45 \mathrm{GeV} \quad \text { or } \quad M_{h^{+}}<70 \mathrm{GeV}
$$

is completely excluded. Our results agree with the conclusions of previous studies on the i2HDM (see, e.g., [29,60]). In particular, the authors of [29] have also stated the $M_{h_{1}}, M_{h_{2}}<45 \mathrm{GeV}$ limit. However, we would like to stress that the general exclusion for $M_{h_{1}}, M_{h_{2}}$ and for $M_{h^{+}}$ given by Eq. (31) is established here for the first time, to the best of our knowledge. In [29], for example, the authors demonstrate [see Fig. 6 and Eq. (18) in [29]] that $M_{h^{+}}$ above $M_{H}$ is excluded from a specific scan. Here we find that $M_{h^{+}}$as light as $70 \mathrm{GeV}$ is allowed by all present constraints, while $M_{h_{1}}$ and $M_{h_{2}}$ are generically allowed to be as light as $45 \mathrm{GeV}$. One should note that specific regions of the parameter space can be excluded using dilepton and missing transverse momentum signatures: for example, in a recent study [73] the authors showed that values of the
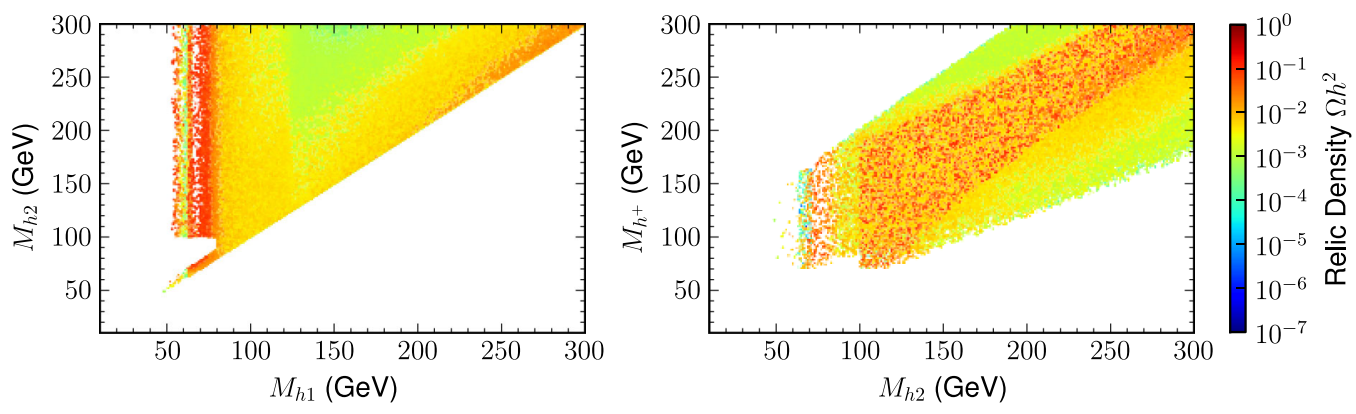

FIG. 7. Color maps of DM relic abundance projected on the planes $\left(M_{h_{1}}, M_{h_{2}}\right)$ and $\left(M_{h_{2}}, M_{h^{+}}\right)$, with all the constraints imposed. 
masses below $M_{h_{1}} \lesssim 50 \mathrm{GeV}$ and $M_{h_{2}} \lesssim 140 \mathrm{GeV}$ can be excluded using this signature, provided that the mass gap between $M_{h_{2}}$ and $M_{h_{1}}$ is large enough. However, we find that this parameter space region is already excluded by the upper cut on the relic density, as one can see from Fig. 7: for $M_{h_{2}}>100 \mathrm{GeV}$, the entire region $M_{h_{1}} \lesssim 50 \mathrm{GeV}$ is excluded by the relic density cut combined with previous constraints including LEPII limits.

\section{B. Fitting the relic density}

In our analysis, we generically allow the DM relic density to be equal or below the PLANCK constraints, Eq. (30). This is the concept of our approach: we assume that in the case of underabundance there should be either additional sources of DM or mechanisms other than thermal freeze-out that compensate for the DM deficit, such as DM freeze-in scenarios [88]. Keeping this in mind, we exclude in our analysis only those regions of the parameter space where the relic density exceeds the PLANCK constraint.

However, it is also instructive to explore the parameter space where both the upper and the lower PLANCK limits are satisfied. This parameter space region is presented in Fig. 8, for a wider scan $10 \mathrm{GeV}<M_{h_{1}}, M_{h_{2}}, M_{h^{+}}<$ $1000 \mathrm{GeV}$ and, separately, for the "zoomed" region $10 \mathrm{GeV}<M_{h_{1}}, M_{h_{2}}, M_{h^{+}}<200 \mathrm{GeV}$. Here, we show the two most revealing 2D projections: $\left(M_{h_{1}}, \lambda_{345}\right)$ and $\left(M_{h_{1}}, M_{h_{2}}\right)$. Additional plots can be found in Appendix.

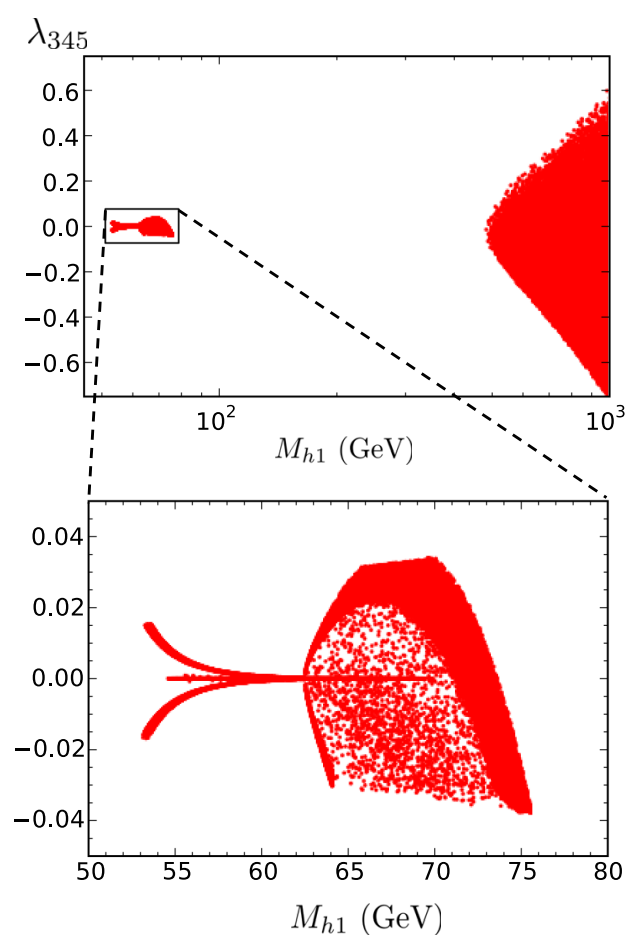

Many interesting features of the $\mathrm{i} 2 \mathrm{HDM}$ parameter space arise once the "correct" amount of DM relic density is required. One observes two very distinct $M_{h_{1}}$ regions: a low mass region for $53 \mathrm{GeV} \lesssim M_{h_{1}} \lesssim 76 \mathrm{GeV}$, shown also in the zoomed panels, and a high mass region for $M_{h_{1}} \gtrsim 490 \mathrm{GeV}$. Below, we discuss them separately.

\section{The low mass region}

In the low mass region we clearly distinguish three regimes with specific physical properties:

(a) A thin horizontal line with very small values of $\lambda_{345}$ can be seen, corresponding to $h_{1} h_{2}$ coannihilation: this region is novel, and it has been missed in previous studies. It can also be seen in the $\left(M_{h_{1}}, M_{h_{2}}\right)$ plots as the thin diagonal strip at low $M_{h_{2}}$ starting from $54 \mathrm{GeV}$ and extending beyond $M_{H} / 2$ up to about $73 \mathrm{GeV}$.

The width of this strip is defined by the maximum allowed value of $\Delta M=M_{h_{2}}-M_{h_{1}}=8 \mathrm{GeV}$, above which the parameter space is excluded by LEP dilepton searches until $M_{h_{2}}>100 \mathrm{GeV}$ [see Eq. (23)]. In this allowed region DM relic density is never above the PLANCK limit given by Eq. (30). The maximum value of $\Omega h_{\mathrm{DM}}^{2}$ reaches the value of about 0.11 for $M_{h_{2}}-M_{h_{1}} \simeq 8 \mathrm{GeV}$ and $\lambda_{345} \simeq 0$, when the only $h_{1}-h_{2}$ coannihilation takes place. For $\Delta M<8 \mathrm{GeV}$ and $M_{h_{1}}<54 \mathrm{GeV}$, the $\Omega h^{2}$ is

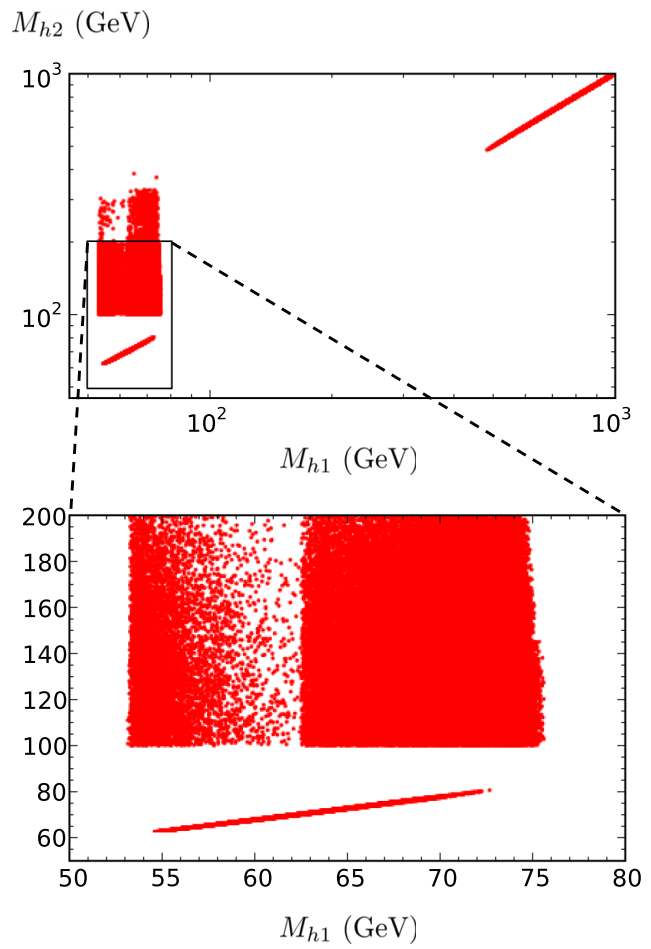

FIG. 8. Projection on two planes of the scan points passing all constraints, and fitting the PLANCK relic abundance within two sigmas. We show both a wide scan with masses between 10 and $1000 \mathrm{GeV}$, and a zoom on the low mass region. 
below the $0.118-2 \times 0.012$ limit which we use in our study because $h_{1}-h_{2}$ coannihilation via $Z$-boson increases with the decrease of $h_{1}, h_{2}$ masses. On the other hand, the upper edge at $73 \mathrm{GeV}$ is defined by the rapid increase of the $h_{1} h_{1} \rightarrow W W^{*}$ contribution, which does not require coannihilation above this mass. The typical $M_{h_{2}}-M_{h_{1}}$ mass split in the coannihilation region is $7-8 \mathrm{GeV}$, as required to make the relic density consistent with the PLANCK limit.

(b) For $M_{h_{1}}<M_{H} / 2$, two symmetric wings can also be seen, extending for positive and negative $\lambda_{345}$, clearly visible in the zoomed $\left(M_{h_{1}}, \lambda_{345}\right)$ panel. They correspond to DM annihilation via the Higgs boson exchange.

(c) In the region $M_{H} / 2<M_{h_{1}} \lesssim 76 \mathrm{GeV}$, large absolute values of $\lambda_{345}$ are allowed by the LHC Higgs data; however, LUX data require $\left|\lambda_{345}\right|$ to be below about 0.04. In this region, we remark on the asymmetric pattern in the $\left(\lambda_{345}, M_{h_{1}}\right)$ plane for positive and negative values of $\lambda_{345}$, which is related, respectively, to the positive and negative interference of $h_{1} h_{1} \rightarrow$ $V V(V=Z, W)$ annihilation diagrams via Higgs boson exchange and diagrams with quartic $h_{1} h_{1} V V$ interactions.

\section{The high mass region and the $\mathrm{LHC}$ sensitivity}

The relic density can also be "just right" at large masses $M_{h_{1}} \gtrsim 490 \mathrm{GeV}$, as shown in Fig. 8. The most salient feature of this high-mass region is the high degree of degeneracy among the three inert Higgs boson masses. This is clearly seen in the upper right corner of the $\left(M_{h_{1}}, M_{h_{2}}\right)$ plane, as well as in Fig. 28 in Appendix. Numerically we find that the maximal mass difference among $h_{1}, h_{2}$, and $h^{+}$, which we call $\Delta M^{\text {max }}$, does not exceed a few GeV.

Remarkably, the mass split is required to be large enough, so that the relic density can reach the lower value of the PLANCK limit: the increase of the mass split is, in fact, correlated with the increase of the quartic coupling $h_{1} h_{1} V_{L} V_{L}$ of the DM to longitudinal $Z$ and $W$ bosons, which enhances the $h_{1} h_{1}$ annihilation cross section, thus bringing the DM relic density down to within the experimental limits. Because of the connection between the mass split and the $h_{1} h_{1} V_{L} V_{L}$ couplings [see Eq. (8)], this effect is actually stronger than the effect of the $h_{1}, h_{2}$, and $h^{+}$ coannihilation, which becomes subdominant in this highmass region. One should also mention that $\Delta M^{\max }$ of the order of a few $\mathrm{GeV}$ is generically not small enough to lead to long-lived $h_{2}$ or $h^{+}$at detector level. However, in the small mass tip, in the interval $550 \mathrm{GeV} \gtrsim M_{h_{1}} \gtrsim 490 \mathrm{GeV}$, $\Delta M^{\text {max }}$ can take values about $0.2 \mathrm{GeV}$. This specific range of the mass split simultaneously provides an $\Omega_{\mathrm{DM}} h^{2}$ consistent with the PLANCK constraint and a lifetime for $h^{+}$large enough to travel about $10 \mathrm{~cm}$ or more in the detector, thus providing disappearing charged track signatures which have been recently explored by CMS [89] Collaboration.

This small $\Delta M$ region deserves a special discussion which we give below. For the mass splitting $\Delta M \simeq$ $0.2 \mathrm{GeV}=200 \mathrm{MeV}$ the $h^{+}$will dominantly decay into $\pi^{+} h_{1}$ as soon as $\Delta M=M_{h^{+}}-M_{h_{1}}>m_{\pi^{+}} \simeq 140 \mathrm{MeV}$. This happens because when the $\Delta M$ is of the order of pion mass, the naive perturbative calculation of $h^{+} \rightarrow h_{1} W^{+*} \rightarrow$ $h_{1} u \bar{d}$ underestimates the width by about 1 order of the magnitude and therefore overestimates the lifetime of $h^{+}$ by the same amount. For proper evaluation of the lifetime which is crucial for the collider phenomenology we have used the nonperturbative $W-\pi$ mixing,

$$
\mathcal{L}_{W \pi}=\frac{g f_{\pi}}{2 \sqrt{2}} W_{\mu}^{+} \partial^{\mu} \pi^{-}+\text {H.c. },
$$

leading to the effective Lagrangian for $h^{+} \rightarrow h_{1} \pi^{+}$interactions, which in momentum space reads as

$$
\mathcal{L}_{h^{+} h_{1} \pi^{-}}=\frac{i g^{2} f_{\pi}}{4 \sqrt{2} M_{W}^{2}}\left(p_{h^{+}}-p_{h_{1}}\right) \cdot p_{\pi}
$$

where $f_{\pi}=130 \mathrm{MeV}$ is the pion decay constant. This effective Lagrangian is represented by the diagram shown in Fig. 9 with the virtual $W$ boson line contracted to pointlike interaction.

From the Lagrangian above one can find the following formula for the $h^{+} \rightarrow h_{1} \pi^{+}$width in the $\Delta M / M \ll 1$ limit:

$$
\Gamma=\frac{g^{4} f_{\pi}^{2}}{64 \pi M_{W}^{4}} \Delta M^{2} \sqrt{\Delta M^{2}-m_{\pi^{+}}^{2}},
$$

where $g$ is the weak coupling constant. In Fig. 10 we present the decay width of $h^{+}$(left) as well as lifetime and decay length (right) which are functions of $\Delta M$ only. One can see that, for $\Delta M$ in the range $140-200 \mathrm{MeV}, h^{+}$will provide a disappearing charged track signature with the length of 100-10 cm, respectively. For $\Delta M$ below the pion mass the width is defined by the $h^{+} \rightarrow W^{+*} h_{1} \rightarrow e^{+} \nu_{e} h_{1}$ process and drops to the level of $10^{-18} \mathrm{GeV}$ or below, meaning that $h^{+}$becomes collider stable and goes through

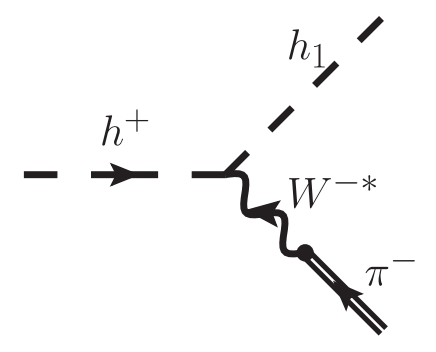

FIG. 9. Feynman diagram representing effective $h^{+} \rightarrow h_{1} \pi^{+}$ via $W-\pi$ mixing. 

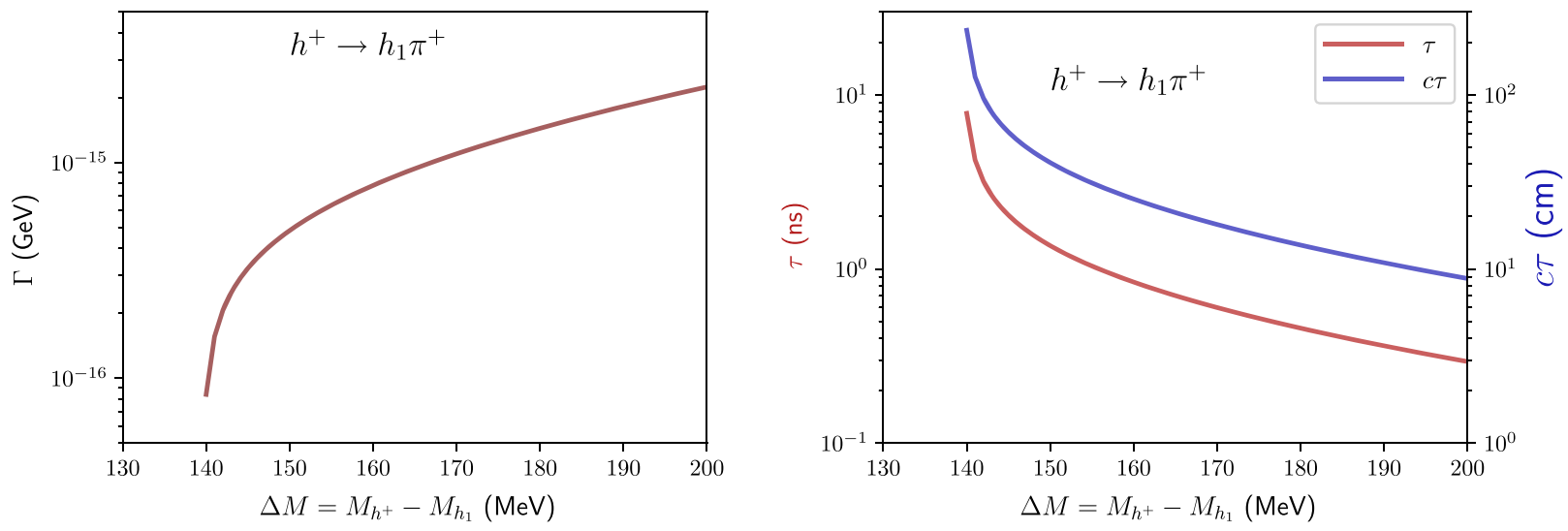

FIG. 10. The decay width of $h^{+}$(left) as well as its lifetime together with the decay length (right).

the whole detector. For such a small mass split, the width of $h^{+}$is proportional to $\Delta M^{5} / M_{W}^{4}$.

The next step is to check which $M_{h^{+}}$mass range in the $\Delta M / M \ll 1$ and $\lambda_{345}$ parameter space is consistent with the relic density constraints given by Eq. (30). Let us stress that in the $\Delta M / M \ll 1$ and $\lambda_{345} \simeq 0$ regime the relic density is maximized for $M_{h^{+}} \simeq 500 \mathrm{GeV}$ or above (this happens because the boost of DM annihilation into the gauge bosons dominates the reduction of co-annihilation effects with $\Delta M / M$ increase). In Fig. 11 we present the $\Omega h^{2}$ value for $\Delta M / M \ll 1$ and $\lambda_{345} \simeq 0$ parameter space which grows with $M_{h^{+}}$and becomes consistent with upper and lower limits for relic density constraints in the range of $490<$ $M_{h^{+}}<600 \mathrm{GeV}$ masses.

At the same time the figure presents the $p p \rightarrow h^{+} h^{-}+$ $h^{ \pm} h_{1,2}$ production rate which we have evaluated for the LHC@8 TeV with NNPDF23LO (as_0119_qed) parton density function (PDF) set [90] and QCD scale chosen to be equal to the averaged transverse mass of the final state particles.

After applying efficiency for the disappearing charged track signatures provided by CMS [89] as a function of

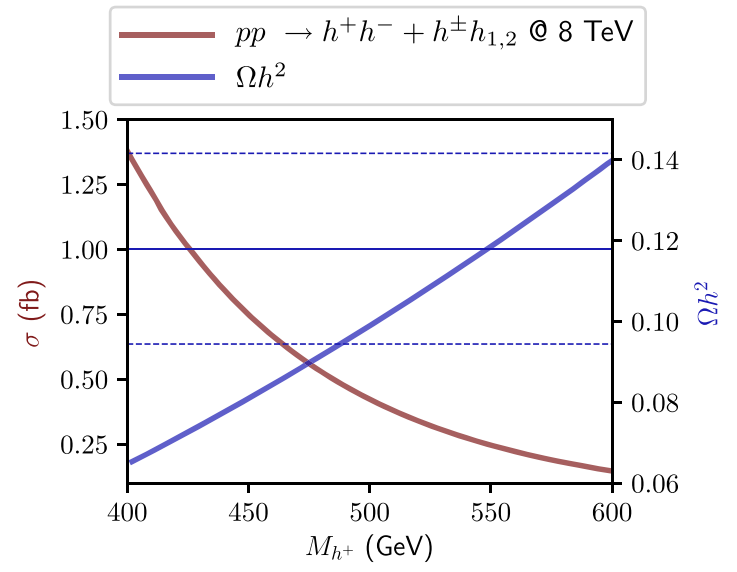

FIG. 11. The $\Omega h^{2}$ value for $\Delta M / M \ll 1$ and $\lambda_{345}$ parameter space and the $p p \rightarrow h^{+} h^{-}+h^{ \pm} h_{1,2}$ production rate evaluated for the LHC@8 TeV. charged track transverse momentum as well as efficiency for distance traveled by the charge particle, we have estimated that CMS@8 TeV with $19.5 \mathrm{fb}^{-1}$ data excludes $h^{+}$in the 490-550 GeV mass range for $\Delta M=$ 140-200 MeV. For example, for $M_{h^{+}}=500 \mathrm{GeV}$ the sum of the cross section of $p p \rightarrow h^{+} h^{-}$and $p p \rightarrow h^{ \pm} h_{1,2}$ is about $0.4 \mathrm{fb}$, and the product of this cross section, the luminosity, and the above efficiencies gives about 2.5 events which are above the 2 event exclusion level.

One should also note that with increasing DM mass, the required split among $h_{1}, h_{2}$, and $h^{+}$increases. At about $20 \mathrm{TeV}$ for $M_{h_{1}}$, the DM relic density constraint together with the requirement of unitarity and perturbativity which are saturated by $\Delta M^{\max } \simeq 10 \mathrm{GeV}$ close the i2HDM parameter space.

\section{PROBING DARK MATTER SIGNALS FROM I2HDM AT THE LHC}

The i2HDM exhibits various signatures that are potentially accessible at the LHC. They can be generically described as "mono-object production," that is, production of several final states in association with large missing transverse momentum. In this section, we undertake a detailed exploration of such processes which goes beyond the previously published state-of-the-art. We will first list the relevant processes, and then produce a cumulative plot which helps us compare their rates. With this knowledge, we will formulate convenient benchmark points which represent various qualitatively distinct regimes of i2HDM, and finally go into a more detailed calculation of monojet production.

\section{A. Dark matter signatures: Diagrams and features}

\section{Monojet production}

The monojet signature originates from the $p p \rightarrow h_{1} h_{1} j$ process, the Feynman diagrams for which are presented in Fig. 12. For this process, the relevant nontrivial parameter space is one dimensional: it is just the DM mass, $M_{h_{1}}$, since 

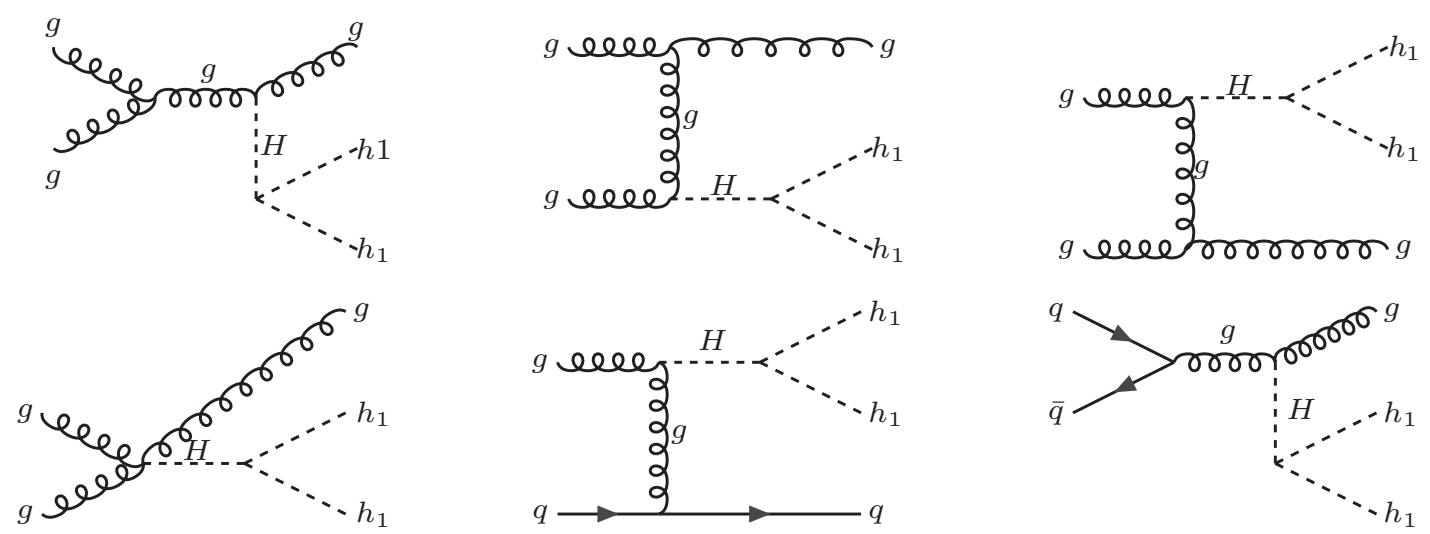

FIG. 12. Feynman diagrams for the $p p \rightarrow h_{1} h_{1} j$ process contributing to a monojet signature.
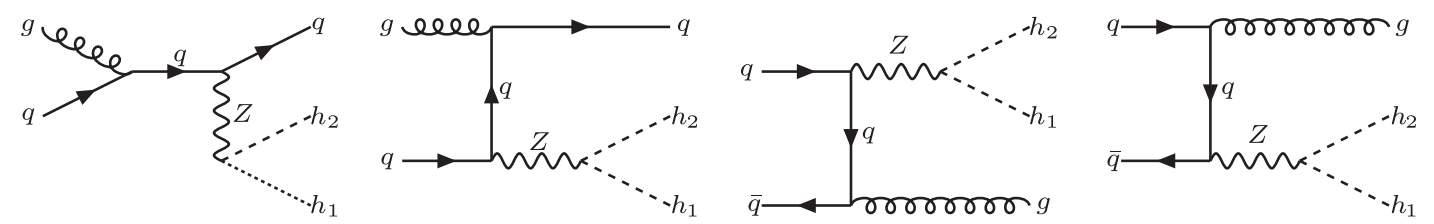

FIG. 13. Feynman diagrams for the $q \bar{q} \rightarrow h_{1} h_{2} g\left(g q \rightarrow h_{1} h_{2} q\right)$ process contributing to a monojet signature.
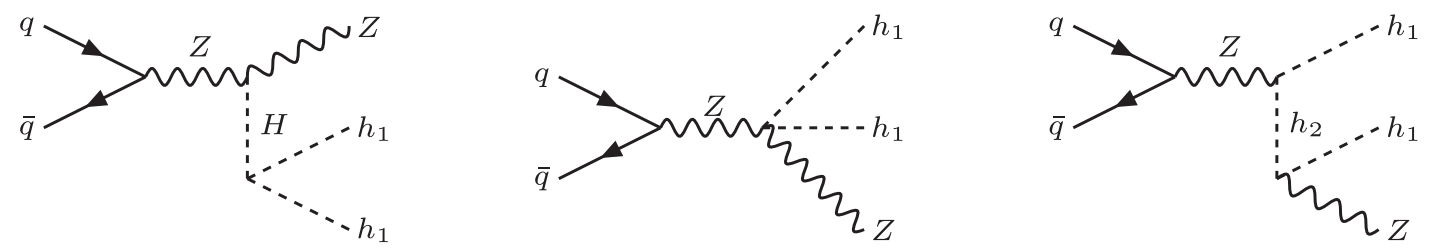

FIG. 14. Feynman diagrams for the $q \bar{q} \rightarrow h_{1} h_{1} Z$ process contributing to a mono- $Z$ signature.

the second parameter, $\lambda_{345}$, simply scales the production cross section which is proportional to $\left(\lambda_{345}\right)^{2}$ for $M_{h_{1}}>M_{H} / 2$. One should note that the mediator mass for this signature is the Higgs mass, $M_{H}=125 \mathrm{GeV}$, thus the EFT approach is not applicable for this process. Also, the recent limits by ATLAS [91] and CMS [92,93] Collaborations are not directly applicable for this process since they have been obtained for a different spin of the mediator and a different spin of DM.

There is one more process, namely $q \bar{q} \rightarrow h_{1} h_{2} g$ $\left(g q \rightarrow h_{1} h_{2} q\right)$ (see diagrams in Fig. 13), that can contribute to a monojet signature in the special case of a small mass split between $h_{1}$ and $h_{2}$. In this scenario $h_{2}$ decays to $h_{1}$ plus soft jets and/or leptons. The essential parameter space for this process is the two-dimensional $\left(M_{h_{1}}, M_{h_{2}}\right)$ plane which fixes its cross section. This channel is particularly relevant in the $\Lambda_{345} \sim 0$ region at low mass that we discovered in this study.

\section{Mono-Z production}

Besides monojets, the i2HDM gives rise to a mono- $Z$ signature, the diagrams for which are presented in Fig. 14.
The first diagram scales with $\lambda_{345}$ while the other two are fixed by electroweak interactions. ${ }^{3}$ In general, nontrivial interference takes place between the three different topologies represented by each of three diagrams, so this process cannot be approximated by a simplified model. However, we found that when $\left|\lambda_{345}\right| \gtrsim 0.02$ with $M_{h_{1}}<M_{H} / 2$ (below the Higgs boson threshold) or $\left|\lambda_{345}\right| \gtrsim 1$ with $M_{h_{1}}>M_{H} / 2$ (above the Higgs boson threshold), the first diagram is dominant and defines the event kinematics. So for these values of $\lambda_{345}$ and $M_{h_{1}}$, a simplified model with the Higgs boson as the mediator is sufficient to set the LHC limits.

One should also note that for values of $\left|\lambda_{345}\right|$ below 0.02 the contribution from diagrams scaling with $\left|\lambda_{345}\right|$ drops below $1 \%$. In this case the $Z$ boson will be the only mediator to probe the $\mathrm{i} 2 \mathrm{HDM}$ model at the LHC, with the mono- $Z$ process being the leading signature for this

\footnotetext{
${ }^{3}$ For the second diagram, the $Z Z h_{1} h_{1}$ vertex for transverse $Z$ bosons is fixed by the weak coupling, while for a longitudinal $Z$ boson it scales with $\tilde{\lambda}_{345}$ in Eq. (8). When this coupling is small, the strength of the $Z Z h_{1} h_{1}$ vertex therefore is fixed by the gauge interactions.
} 
purpose (and not only as a probe complementary to the monojet signature). This signature will be especially pronounced if $M_{h_{2}}-M_{h_{1}}>M_{Z}$, so that the cross section of the mono- $Z$ signature is essentially defined by the cross section of the $2 \rightarrow 2$ process, $p p \rightarrow h_{1} h_{2} \rightarrow h_{1} h_{1} Z$. For small mass split, as it happens in the $\lambda_{345} \sim 0$ region, the mono- $Z$ will complement the signal in the monojet described above. The parameter space for this process is the two-dimensional $\left(M_{h_{1}}, M_{h_{2}}\right)$ plane.

\section{Mono-Higgs production}

The i2HDM could also provide a mono-Higgs signature via $g g \rightarrow h_{1} h_{1} H$ and $q \bar{q} \rightarrow h_{1} h_{2} H$, whose diagrams are presented in Figs. 15 and 16, respectively. The only mediator for $g g \rightarrow h_{1} h_{1} H$ is the Higgs boson, and the respective cross section scales as $\left(\lambda_{345}\right)^{2}$ for small values of $\lambda_{345}$ and $\left(\lambda_{345}\right)^{4}$ for large values of $\lambda_{345}$ because of the second diagram. On the other hand, the $q \bar{q} \rightarrow h_{1} h_{2} H$ process takes place via either a $Z$ boson or an $h_{2}$ as a mediator: the first diagram does not scale with $\lambda_{345}$, while the last two do. Therefore for large $\lambda_{345}$, the $\left(\lambda_{345}\right)^{2}$ scaling takes place for the $q \bar{q} \rightarrow h_{1} h_{2} H$ process. In fact, the contribution from the second and the third diagrams of $q \bar{q} \rightarrow h_{1} h_{2} H$ to the total cross section drops below $1 \%$ only for $\lambda_{345}<0.002$, below which the process kinematics and the cross section are determined by the first diagram with two Z-boson propagators.

\section{Vector boson fusion}

Finally, one should mention the production of DM via vector boson fusion, $p p \rightarrow h_{1} h_{1} j j$, the diagrams for which are presented in Fig. 17. Similar to the mono- $Z$ process, there
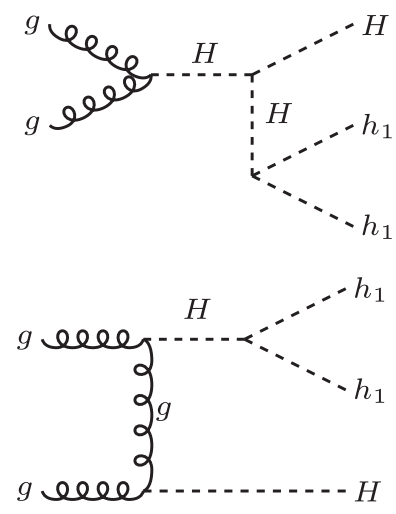
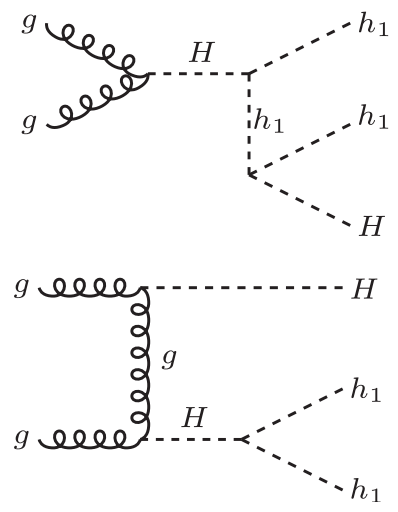
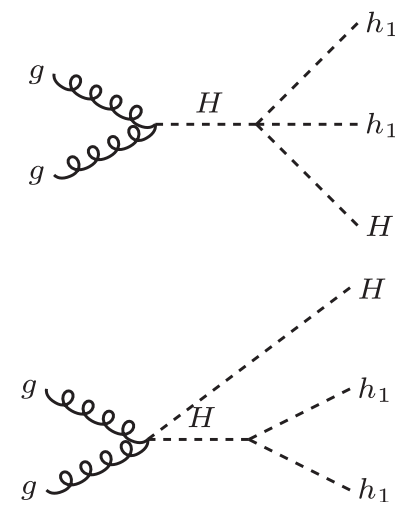

FIG. 15. Feynman diagrams for the $g g \rightarrow h_{1} h_{1} H$ process contributing to a mono-Higgs signature.
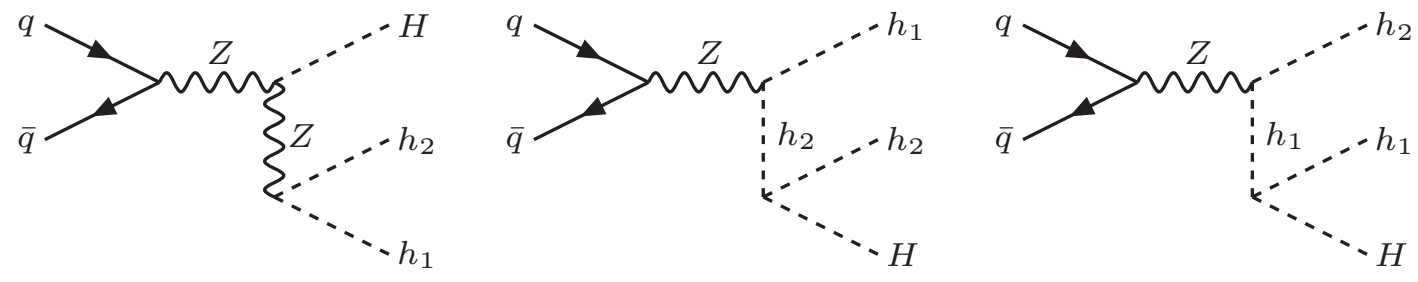

FIG. 16. Feynman diagrams for the $q \bar{q} \rightarrow h_{1} h_{2} H$ process contributing to a mono-Higgs signature.
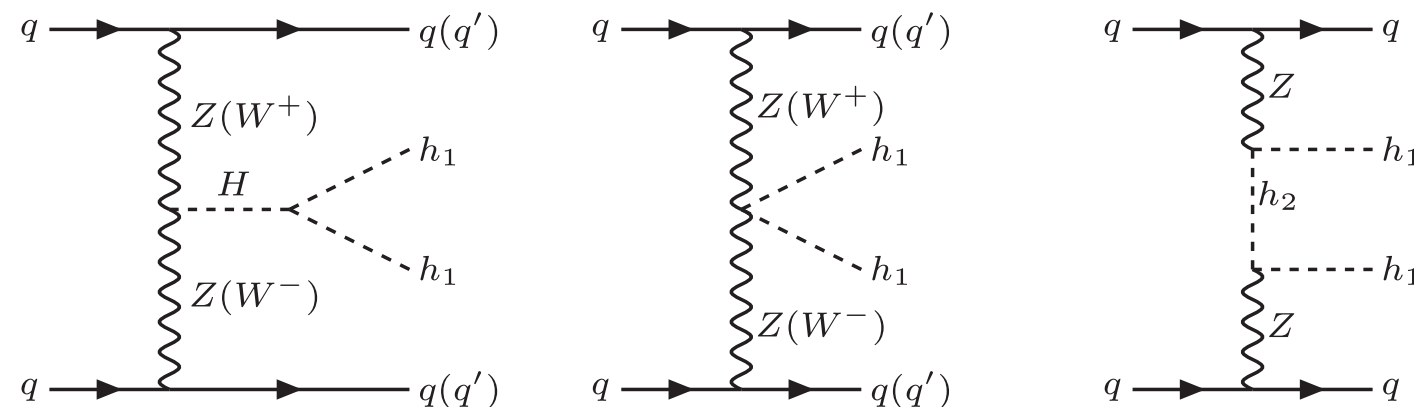

FIG. 17. Diagrams for $q q \rightarrow q^{\left({ }^{\prime}\right)} q^{\left({ }^{\prime}\right)} h_{1} h_{1}$ DM production in the vector boson fusion process. 
are three diagrams with different topologies and mediators which contribute to this process; thus, it cannot be described by just one simplified model. The first two diagrams scale with $\lambda_{345}$. To be accurate, the $Z_{L} Z_{L} h_{1} h_{1}$ coupling in the second diagram is proportional to $\tilde{\lambda}_{345}$ [see Eq. (8)], which is approximately equal to $\lambda_{345}$ for small $M_{h_{2}}-M_{h_{1}}$. They give the dominant contribution to the $p p \rightarrow h_{1} h_{1} j j$ process for $\lambda_{345} \simeq 1$, but their contribution is negligible with very small $\lambda_{345}$. On the other hand, for large $h_{1}-h_{2}$ and $h_{1}-h^{+}$ splittings, they get stronger even with small $\lambda_{345}$ and enhance the vector boson fusion (VBF) process. This opens a new perspective for the exploration of the i2HDM model which we plan to perform in the near future.

\section{B. Mono-object production: Rates and comparison}

\section{Implementation and cuts}

When calculating the cross sections of mono-object production at the LHC, we used the following setup for the process evaluation:

(i) the QCD renormalization and factorization scales $Q$ were chosen to be equal to the transverse momentum of the pair of DM particles, i.e. missing transverse momentum, $\boldsymbol{E}_{\mathrm{T}}^{\text {miss }}$ for all processes;

(ii) the PDF and the strong coupling constant are as provided by the NNPDF23LO (as_0119_qed) PDF set [90];

(iii) for all processes a cut on the minimal value of missing transverse momentum of $100 \mathrm{GeV}$ was applied;

(iv) the VBF cross section has been evaluated with the following additional cuts:

$$
P_{T}^{j}>30 \mathrm{GeV}, \quad \Delta \eta_{j j}>4, \quad E_{j}>400 \mathrm{GeV} .
$$

Below we present plots and numbers for cross sections (in the text and table) with three significant digits corresponding to the accuracy of the $\mathrm{MC}$ phase space integration. But we would like to note that when $Q$ is varied in the range $\boldsymbol{E}_{\mathrm{T}}^{\text {miss }} / 2$ to $2 \times \boldsymbol{E}_{\mathrm{T}}^{\text {miss }}$, the QCD scale uncertainty is around 20\%-30\% for the tree-level cross sections presented, dominating over PDF uncertainties which are below $10 \%$. The presentation and detailed discussion of these uncertainties is out of the scope of this paper.

\section{Production rates}

In Fig. 18 we present a summary of the cross sections versus dark matter mass $M_{h_{1}}$ for all the processes mentioned above, which contribute to the monojet, mono- $Z$, mono-Higgs, and VBF signatures for the LHC at 8 and $13 \mathrm{TeV}$. In the plot we chose some particular values of the mass split $M_{h_{2}}-M_{h_{1}}$ and of the coupling $\lambda_{345}$; however, the cross sections in other cases can easily be inferred by referring to the scaling properties mentioned in Sec. IV A. It is evident from the plot that the dominant cross sections always tend to be monojet processes (in grey), and the mono- $Z$ signature coming from the on-shell decay of $h_{2}$.

It is also worth focusing on the new $\lambda_{345} \sim 0$ region at small masses: the relevant cross sections are related to the mono-jet process $p p \rightarrow j h_{1} h_{2}$ at a small mass split (dashed grey curves in Fig. 18) and mono-Higgs production (dotted red curves). In the low $M_{h_{1}}$ region, below $75 \mathrm{GeV}$, the former channel provides cross sections of several tens of $\mathrm{fb}$ at $13 \mathrm{TeV}$, which may be probed in monojet searches. This is a very important point, as the $\lambda_{345} \sim 0$ region is very difficult to probe in DD experiments due to the suppressed coupling to the Higgs.

The plot also shows that the mono-Higgs channels does not yield detectable rates.

The process $p p \rightarrow j h_{1} h_{2}$ is also relevant for larger mass splits: while the cross section drops, for splits of few tens of $\mathrm{GeV}$, the $h_{2}$ decays can provide visible leptons and thus produce Supersymmetry-like signatures. Finally, when the mass split grows above the $Z$ mass, on-shell decays of $h_{2}$ allow for mono- $Z$ signatures, as shown by the blue-dashed line.

\section{Benchmark points}

The experience we have gained so far, in both relic density and mono-object cross section calculations, allows us to discern several qualitatively distinct regimes of i2HDM and find their representative benchmark points. In Table I we present six benchmarks (BM) from the i2HDM parameter space together with corresponding observables: DM relic density $\left(\Omega_{\mathrm{DM}} h^{2}\right)$, spin-independent DM scattering rate on the proton $\left(\sigma_{\mathrm{SI}}^{p}\right)$ accompanied with its ratio to the experimental limit from LUX following rescaling with the relic density: $R_{\mathrm{SI}}^{\mathrm{LUX}}=$ $\left(\sigma_{\mathrm{SI}}^{p} / \sigma_{\mathrm{SI}}^{\mathrm{LUX}}\right) \cdot\left(\Omega_{\mathrm{DM}} / \Omega_{\mathrm{DM}}^{\text {Planck }}\right)$. We also present the LHC cross sections for the monojet, mono- $Z$, and mono- $H$ signatures discussed above with a $\boldsymbol{E}_{\mathrm{T}}^{\text {miss }}>100 \mathrm{GeV}$ cut applied. All of these benchmarks are allowed by the present experimental data. In Table I we do not give specific benchmarks for the long-lived $h^{+}$scenario discussed above since in Fig. 11 we have effectively provided the whole parameter space for this scenario $(\Delta M=140-200 \mathrm{MeV}$, $\lambda_{345} \simeq 0, M_{h^{+}}=490-600 \mathrm{GeV}$ ).

The first two benchmarks have small and medium values of $\lambda_{345}$ and correspond to the scenario when $M_{h_{1}}$ is below $M_{H} / 2$, and the mass split $\Delta M=M_{h_{2}}-M_{h_{1}}$ is small. BM1 has a very small value of $\lambda_{345}=10^{-4}$ and is therefore characterized by having a small $\operatorname{Br}\left(H \rightarrow h_{1} h_{1}\right)$ value and a very low DM direct detection rate, $\sigma_{\mathrm{SI}}^{p}$, while the relic density is consistent with the Planck limit due to coannihilation. The $h_{1} h_{1} j$ monojet signature rate at the LHC scales with $\left(\lambda_{345}\right)^{2}$ and is therefore very low, while the $\lambda_{345}$-independent $h_{1} h_{2} j$ signature cross section is about $36.7 \mathrm{fb}$ (LHC@8 TeV) and $92.4 \mathrm{fb}$ (LHC@13 TeV). 

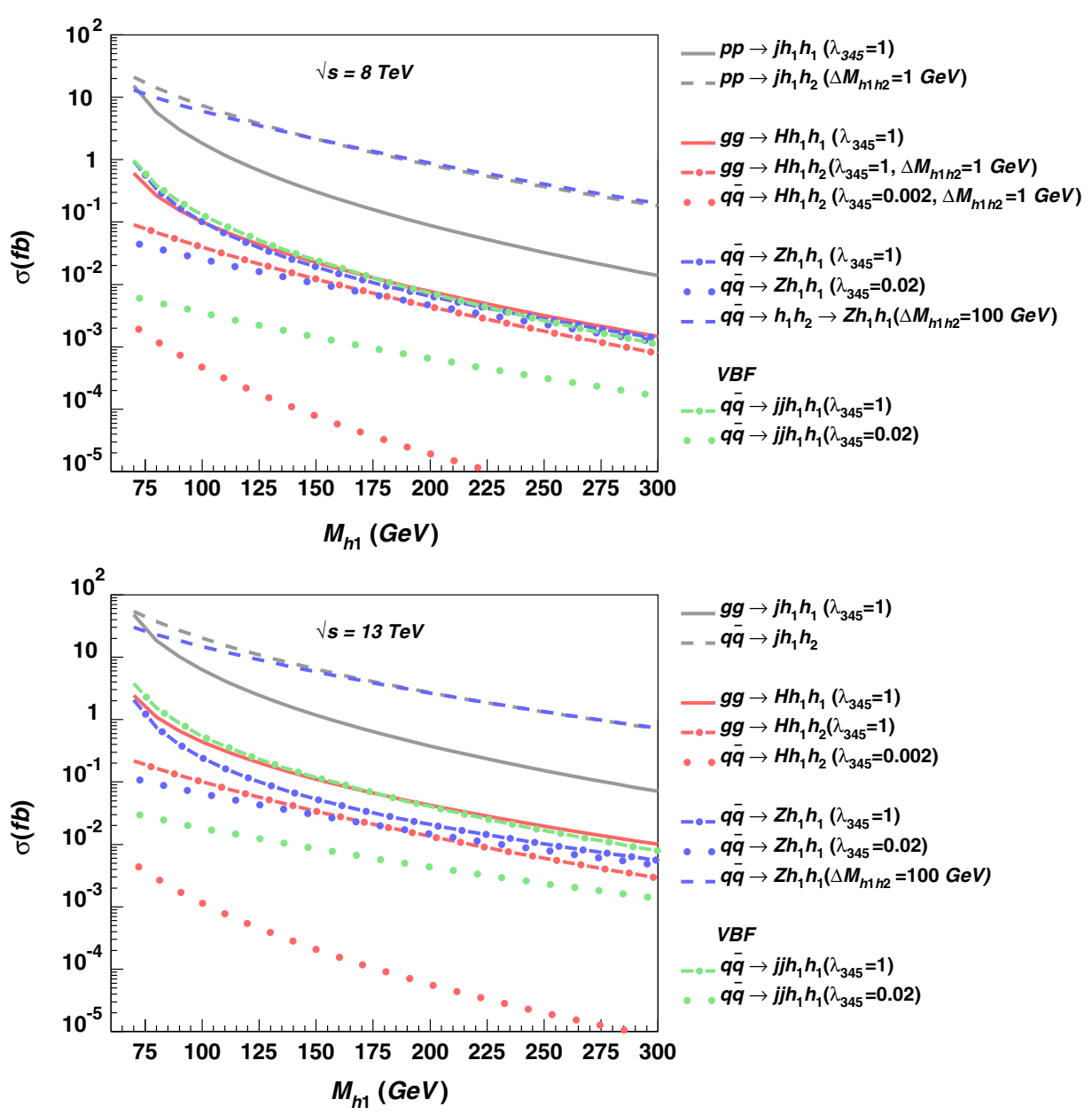

FIG. 18. Cross sections versus dark matter mass, $M_{h_{1}}$, for processes contributing to monojet, mono-Z, mono-Higgs, and VBF signatures for the LHC@8 TeV and LHC@13 TeV.

BM2 differs from BM1 only by the value of $\lambda_{345}=0.027$, which is chosen as the maximum value allowed by the Higgs invisible branching ratio. For this $\lambda_{345}$, the $h_{1} h_{1} j$ monojet production rates are $288 \mathrm{fb}$ (LHC@8 TeV) and 878 fb (LHC@13 TeV).

$\mathrm{BM} 3$ and BM4 correspond to the scenarios where $\Delta M>M_{Z}$ with $M_{h_{1}}$ below and above $M_{H} / 2$, respectively, with the other parameters chosen such that the relic density is consistent with Planck data. In comparison to BM3, BM4 has a very low $h_{1} h_{1} j$ production cross section because the SM Higgs boson is produced off mass shell. At the same time the $h_{1} h_{1} Z$ cross section is of the same order for both benchmarks: $6.48 \mathrm{fb}$ and $3.90 \mathrm{fb}$ for LHC@8 TeV, and $17.8 \mathrm{fb}$ and $11.1 \mathrm{fb}$ for LHC@13 TeV, respectively.

Finally, BM5 and BM6 represent the cases with a small $(5 \mathrm{GeV})$ mass split and $M_{h_{1}}=100 \mathrm{GeV}$. The only difference in the input parameters is the value of $\lambda_{345}$ : large $\lambda_{345}=1$ for BM5 and small $\lambda_{345}=0.002$ for BM6. For both benchmarks, the DM relic density is well below the PLANCK limit, and therefore an additional source of dark matter is required. Even for BM6 which has a small value of $\lambda_{345}$, the DM relic density is of the order of $10^{-3}$ because the DM effectively annihilate via $h_{1} h_{1} \rightarrow V V$ and $h_{1} h_{1} \rightarrow$ $H H$ channels. They are open for this value of DM mass and are defined essentially by the weak coupling, the contribution from $h_{1} h_{1} \rightarrow V_{L} V_{L}$ being small because of the small $h_{1}-h_{2}$ mass split and the contribution from coannihilation being subdominant for this value of mass split. For both of these benchmarks, the $h_{1} h_{2} j$ channel which has cross sections of $6.93 \mathrm{fb}$ (LHC@8 TeV) and $19.1 \mathrm{fb}$ (LHC@13 TeV) looks the most promising.

From Table I one can see that different mono-object signatures are very complementary for these suggested benchmarks, especially the $h_{1} h_{1} j$ and $h_{1} h_{2} j$ processes which are the main focus of the collider study presented below.

\section{Limits from LHC@8 TeV and projections for LHC@13 TeV}

In the previous subsections, we calculated the monoobject production cross sections at the LHC as a function of DM mass $M_{h_{1}}$ for a selection of parameters. In this subsection, we invert the problem: we examine the limits on the parameter space which follow from the current 
TABLE I. Benchmarks (BM) from the i2HDM parameter space together with corresponding observables: DM relic density $\left(\Omega_{\mathrm{DM}} h^{2}\right)$, spin-independent DM scattering rate on the proton $\left(\sigma_{\mathrm{SI}}^{p}\right)$ accompanied with its ratio to the experimental limit from LUX following rescaling with the relic density: $R_{\mathrm{SI}}^{\mathrm{LUX}}=\left(\sigma_{\mathrm{SI}}^{p} / \sigma_{\mathrm{SI}}^{\mathrm{LUX}}\right) \cdot\left(\Omega_{\mathrm{DM}} / \Omega_{\mathrm{DM}}^{\text {Planck }}\right)$, and the LHC cross sections for monojet, mono- $Z$, and mono- $H$ signatures with a $\boldsymbol{E}_{\mathrm{T}}^{\mathrm{miss}}>100 \mathrm{GeV}$ cut applied.

\begin{tabular}{|c|c|c|c|c|c|c|}
\hline BM & 1 & 2 & 3 & 4 & 5 & 6 \\
\hline$M_{h_{1}}[\mathrm{GeV}]$ & 55 & 55 & 50 & 70 & 100 & 100 \\
\hline$M_{h_{2}}[\mathrm{GeV}]$ & 63 & 63 & 150 & 170 & 105 & 105 \\
\hline$M_{h_{+}}[\mathrm{GeV}]$ & 150 & 150 & 200 & 200 & 200 & 200 \\
\hline$\lambda_{345}$ & $1.0 \times 10^{-4}$ & 0.027 & 0.015 & 0.02 & 1.0 & 0.002 \\
\hline$\lambda_{2}$ & 1.0 & 1.0 & 1.0 & 1.0 & 1.0 & 1.0 \\
\hline$\Omega_{\mathrm{DM}} h^{2}$ & $9.2 \times 10^{-2}$ & $1.5 \times 10^{-2}$ & $9.9 \times 10^{-2}$ & $9.7 \times 10^{-2}$ & $1.3 \times 10^{-4}$ & $1.7 \times 10^{-3}$ \\
\hline$\sigma_{\mathrm{SI}}^{p}[\mathrm{pb}]$ & $1.7 \times 10^{-14}$ & $1.3 \times 10^{-9}$ & $4.8 \times 10^{-10}$ & $4.3 \times 10^{-10}$ & $5.3 \times 10^{-7}$ & $2.1 \times 10^{-12}$ \\
\hline$R_{\mathrm{SI}}^{\mathrm{LUX}}$ & $1.6 \times 10^{-5}$ & 0.19 & 0.51 & 0.37 & 0.48 & $2.5 \times 10^{-5}$ \\
\hline $\operatorname{Br}\left(H \rightarrow h_{1} h_{1}\right)$ & $5.2 \times 10^{-6}$ & 0.27 & 0.13 & 0.0 & 0.0 & 0.0 \\
\hline \multicolumn{7}{|l|}{$\sigma_{\mathrm{LHC} 8}[\mathrm{fb}]$} \\
\hline$h_{1} h_{1} j$ & $5.44 \times 10^{-3}$ & 288 & 134 & $6.05 \times 10^{-3}$ & 1.80 & $7.23 \times 10^{-6}$ \\
\hline$h_{1} h_{2} j$ & 36.7 & 36.7 & 6.48 & 3.90 & 6.93 & 6.93 \\
\hline$h_{1} h_{1} Z$ & $6.14 \times 10^{-2}$ & 21.4 & 30.7 & 12.2 & 0.101 & $2.52 \times 10^{-2}$ \\
\hline$h_{1} h_{1} H$ & $1.70 \times 10^{-4}$ & 8.98 & 4.21 & $2.19 \times 10^{-4}$ & 0.100 & $3.33 \times 10^{-7}$ \\
\hline$h_{1} h_{2} H$ & $5.35 \times 10^{-3}$ & $6.31 \times 10^{-3}$ & $9.80 \times 10^{-3}$ & $7.54 \times 10^{-3}$ & $3.86 \times 10^{-2}$ & $5.51 \times 10^{-4}$ \\
\hline$h_{1} h_{1} j j$ & $2.39 \times 10^{-2}$ & 17.2 & 8.11 & $4.44 \times 10^{-2}$ & 0.212 & $1.62 \times 10^{-2}$ \\
\hline \multicolumn{7}{|l|}{$\sigma_{\mathrm{LHC13}}[\mathrm{fb}]$} \\
\hline$h_{1} h_{1} j$ & $1.67 \times 10^{-2}$ & 878 & 411 & $1.93 \times 10^{-2}$ & 6.25 & $2.50 \times 10^{-5}$ \\
\hline$h_{1} h_{2} j$ & 92.4 & 92.4 & 17.8 & 11.1 & 19.1 & 19.1 \\
\hline$h_{1} h_{1} Z$ & 0.153 & 46.2 & 66.9 & 28.3 & 0.241 & $6.47 \times 10^{-2}$ \\
\hline$h_{1} h_{1} H$ & $6.69 \times 10^{-4}$ & 35.3 & 16.5 & $9.08 \times 10^{-4}$ & 0.441 & $1.51 \times 10^{-6}$ \\
\hline$h_{1} h_{2} H$ & $1.18 \times 10^{-2}$ & $1.40 \times 10^{-2}$ & $2.47 \times 10^{-2}$ & $1.99 \times 10^{-2}$ & $9.82 \times 10^{-2}$ & $1.34 \times 10^{-3}$ \\
\hline$h_{1} h_{1} j j$ & 0.101 & 62.7 & 29.6 & 0.189 & 0.904 & $7.49 \times 10^{-2}$ \\
\hline
\end{tabular}

$8 \mathrm{TeV}$ and projected $13 \mathrm{TeV}$ LHC data. We concentrate on limits from monojet processes, as these are the mono-object signatures with the highest cross sections, as shown in Fig. 18. For monojet signals we consider two different processes: $p p \rightarrow h_{1} h_{1} j$ and $p p \rightarrow h_{1} h_{2} j$. The cross section of the former depends on the two parameters only, the dark matter mass $M_{h_{1}}$ and $\lambda_{345}$. For the latter, all the vertices depend only on the gauge constants. The only two parameters that shape its cross section are the inert scalar masses $M_{h_{1}}$ and $M_{h_{2}}$, or equivalently $M_{h_{1}}$ and $\Delta M=$ $M_{h_{2}}-M_{h_{1}}$.

\section{Implementation and the LHC data used}

In order to calculate the limits from the LHC at $8 \mathrm{TeV}$, we used the CHECKMATE [94-102] framework, which allows an easy application of the implemented search analyses. This tool takes a given sample of Monte Carlo events in the HEP or HepMC format after parton showering and hadronization, for which we used PYTHIA-6 [103], and performs a detector simulation on these events using DeLPHES-3 [95]. Subsequently CHECKMATE can apply any of its preprogrammed and validated analyses to the generated signal events and uses the resulting efficiencies along with published information, such as the $95 \%$ confidence level limit on signal count, to produce results from which we can find the cross section limit placed on our model by each analysis.

The signature of both processes that we consider, $p p \rightarrow$ $h_{1} h_{1} j$ and $p p \rightarrow h_{1} h_{2} j$, is a high- $p_{T}$ jet and a large missing transverse momentum, $\boldsymbol{E}_{\mathrm{T}}^{\mathrm{miss}}$. In the case of $p p \rightarrow h_{1} h_{2} j$, the $h_{2}$ will decay via a $h_{1}$ and a $Z^{(*)}$ boson. When $\Delta M$ is very small, the decay products of the $Z$ will generally be too soft to be reconstructed in the detector. Therefore in this case $p p \rightarrow h_{1} h_{2} j$ will give a monojet $+\boldsymbol{E}_{\mathrm{T}}^{\text {miss }}$ signature. Using CHескMATE and HepMC files created with the i2HDM model implemented in CALCHEP, we calculated the limits given by all of the monojet $+\boldsymbol{E}_{\mathrm{T}}^{\text {miss }}$ analyses currently implemented in CHECKMATE [93,104-106] (3 ATLAS and 1 CMS analyses).

For both processes considered, we found that the lowest cross section limits for each benchmark point considered were provided by one of the ATLAS monojet $+\boldsymbol{E}_{\mathrm{T}}^{\text {miss }}$ analyses [106]. These are the limits presented in this section. This analysis requires a leading jet with a $p_{T}>120 \mathrm{GeV}$ and $|\eta|<2.0$, and the leading jet $p_{T} / \boldsymbol{E}_{\mathrm{T}}^{\mathrm{miss}}>0.5$. Furthermore, to reduce the multijet background where the large $\boldsymbol{E}_{\mathrm{T}}^{\text {miss }}$ originates mainly from the jet energy mismeasurement, we place a requirement 
on the azimuthal separation $\Delta \phi\left(\right.$ jet, $\left.p_{T}^{\text {miss }}\right)>1.0$ between the direction of the missing transverse momentum and that of each jet. A number of different signal regions are considered with increasing $\boldsymbol{E}_{\mathrm{T}}^{\text {miss }}$ thresholds from 150 to $700 \mathrm{GeV}$. Full details are available in the ATLAS paper [106].

In order to project these limits for increased luminosity and to $13 \mathrm{TeV}$, we use Monte Carlo events to estimate the efficiencies for the signal and background at $13 \mathrm{TeV}$, which is a function of $M_{h_{1}}$ and depends on the best analysis signal region for each mass. We make the assumption that the analysis cuts for $13 \mathrm{TeV}$ data will be the same as for $8 \mathrm{TeV}$ data, which does not take into account improvements in the signal to background ratio which would likely occur with new analysis cuts at $13 \mathrm{TeV}$. Therefore our projected limits will be slightly conservative.

\section{Limits from $p p \rightarrow h_{1} h_{1} j$}

The results for the process $p p \rightarrow h_{1} h_{1} j$ are shown for $8 \mathrm{TeV}$ in Fig. 19(a) with projections to $13 \mathrm{TeV}$ and higher luminosities in Fig. 19(b). The limits are denoted by the solid lines, while the cross sections for the i2HDM for different values of $\lambda_{345}$ are shown by the dashed lines. For $M_{h_{1}}<M_{H} / 2$, the maximum allowed value of $\lambda_{345}$ is given by the bound on the invisible Higgs branching ratio in Eq. (25) (this constraint has not been applied on the dashed blue $\lambda_{345}=0.1$ curve), while when $M_{h_{1}}>M_{H} / 2$ the maximum allowed value is calculated using the constraints of Eq. (17). The cross section with this maximum value of $\lambda_{345}$ is denoted by the dashed green line. We see in Fig. 19(a), that the $8 \mathrm{TeV}$ LHC monojet $+\boldsymbol{E}_{\mathrm{T}}^{\text {miss }}$ searches do not constrain the i2HDM via the $p p \rightarrow h_{1} h_{1} j$ process. However, at $13 \mathrm{TeV}$, shown in Fig. 19(b), with around
$100 \mathrm{fb}^{-1}$ of data (purple solid curve), we would be able to set limits on $\lambda_{345}$ for $M_{h_{1}}$ up to $66 \mathrm{GeV}$, and for $3000 \mathrm{fb}^{-1}$ (black solid curve) LHC data would set limits on $\lambda_{345}$ for $M_{h_{1}}$ up to $83 \mathrm{GeV}$. It should be remarked that the spike in cross section on the green dashed line at $M_{h_{1}} \sim M_{H} / 2$ is due to the release of the ( $H \rightarrow$ invisible) bound on $\lambda_{345}$ once the decay of the Higgs into DM is kinematically closed.

We should note that a similar projection of CMS monojet limits [93] at $14 \mathrm{TeV}$ has been studied previously [60], where the projected limits were slightly stronger than in Fig. 19(b). Their projection was able to limit $M_{h_{1}}$ for values of $\lambda_{345}$ as small as $\lambda_{345}=0.01$, while we require slightly larger values of $\lambda_{345}$ in order to limit $M_{h_{1}}$. We would like to note that in our paper the limits are based on the fast detector simulations rather than parton level ones used in [60] done for $14 \mathrm{TeV}$. Taking this into account we consider our results as a more realistic projection of the future LHC data potential.

In Fig. 20 we provide the limit on $\lambda_{345}$ versus $M_{h_{1}}$ for different projected luminosities at the LHC@13 TeV. This limit is derived from the analysis presented in Fig. 19 and could be more practical for comparison with limits on $\lambda_{345}$ from different experiments.

\section{Limits from $p p \rightarrow h_{1} h_{2} j$}

For $p p \rightarrow h_{1} h_{2} j$, the results are shown in Fig. 21(a) for $8 \mathrm{TeV}$ and in Fig. 21(b) for $13 \mathrm{TeV}$. We consider two scenarios with a small ( $\Delta M=1 \mathrm{GeV}$ in blue curves) and large $(\Delta M=100 \mathrm{GeV}$ in yellow curves) mass split. The projected cross section limits are again denoted by the solid lines. When $\Delta M=1 \mathrm{GeV}$, the current LHC Run I results are able to rule out $M_{h_{1}}<35 \mathrm{GeV}$. In this case, it should be

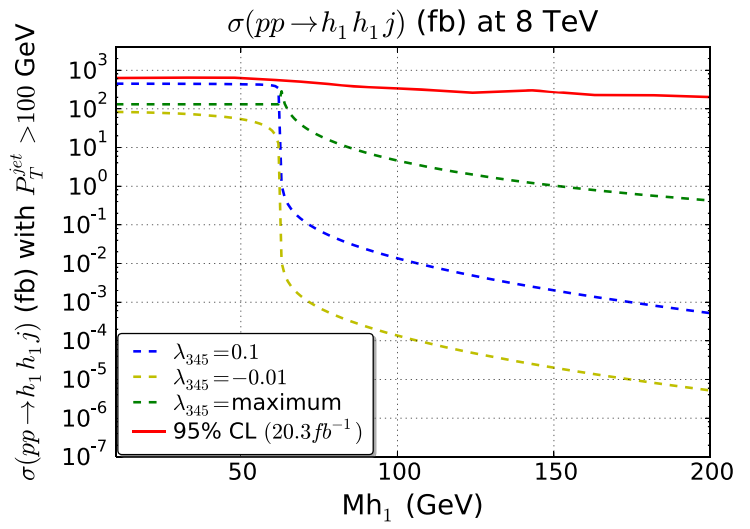

(a) $M_{h_{1}}$ vs cross section at $8 \mathrm{TeV}$.

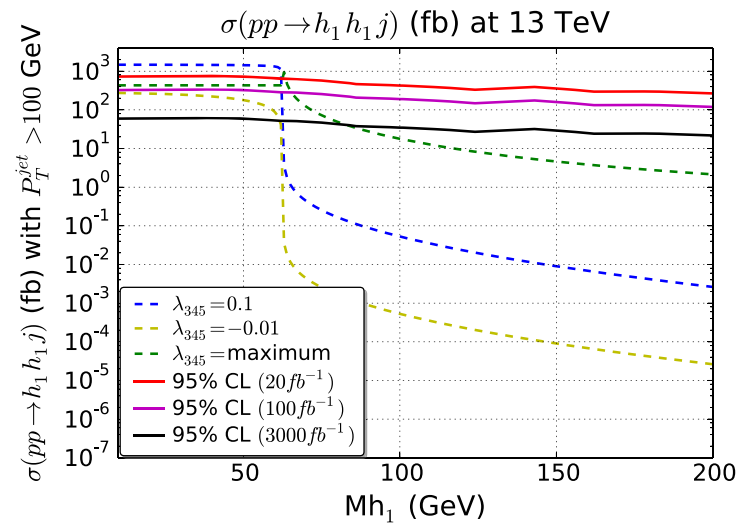

(b) $M_{h_{1}}$ vs cross section at $13 \mathrm{TeV}$.

FIG. 19. Cross sections and 95\% C.L.s for $p p \rightarrow h_{1} h_{1} j$ versus $M_{h_{1}}$ at 8 and $13 \mathrm{TeV}$. In both cases, the cross sections are shown for three different values of $\lambda_{345}$ : (i) $\lambda_{345}=0.1$ (blue dashed curve), (ii) $\lambda_{345}=-0.01$ (yellow dashed curve), and (iii) the maximum $\lambda_{345}$ value (green dashed curve) allowed by constraints (described in text). (a) Results for $8 \mathrm{TeV}$, with limits (solid red curve) calculated using the ATLAS analysis [106]. (b) Results for $13 \mathrm{TeV}$, with projected limits for the ATLAS analysis [106] with luminosities of 20 $\mathrm{fb}^{-1}$, $100 \mathrm{fb}^{-1}$, and $3000 \mathrm{fb}^{-1}$ (red, magenta, and black solid lines) at $13 \mathrm{TeV}$. 


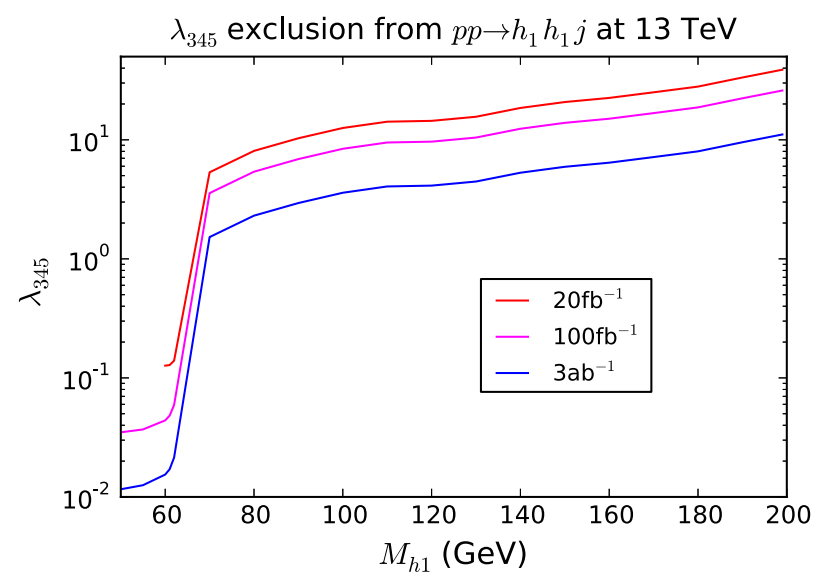

FIG. 20. Projected limit on $\lambda_{345}$ from $p p \rightarrow h_{1} h_{1} j$ at $13 \mathrm{TeV}$ derived from the analysis presented in Fig. 19.

emphasized that as the couplings of the relevant diagrams (see Fig. 13) are fixed by the gauge couplings, this limit on $M_{h_{1}}$ is independent of all parameters other than $\Delta M$. At $13 \mathrm{TeV}$, and at higher luminosities, this lower limit on $M_{h_{1}}$ in this degenerate mass scenario is improved slightly to $41 \mathrm{GeV}, 43 \mathrm{GeV}$, and $55 \mathrm{GeV}$ for $20 \mathrm{fb}^{-1}$ (solid red curve), $100 \mathrm{fb}^{-1}$ (solid magenta curve), and $3000 \mathrm{fb}^{-1}$ (solid black curve) of integrated luminosity, respectively, as is shown in Fig. 21(b). For $\Delta M=100 \mathrm{GeV}$, the production cross section is much smaller and the model is not constrained via monojet and $\boldsymbol{E}_{\mathrm{T}}^{\mathrm{miss}}$ signatures from the $p p \rightarrow h_{1} h_{2} j$ process. However, in this region other collider signatures such as dilepton $+\boldsymbol{E}_{\mathrm{T}}^{\text {miss }}$ from the decay $h_{2} \rightarrow h_{1} Z$ are available and will provide stronger limits as studied for example in [73].

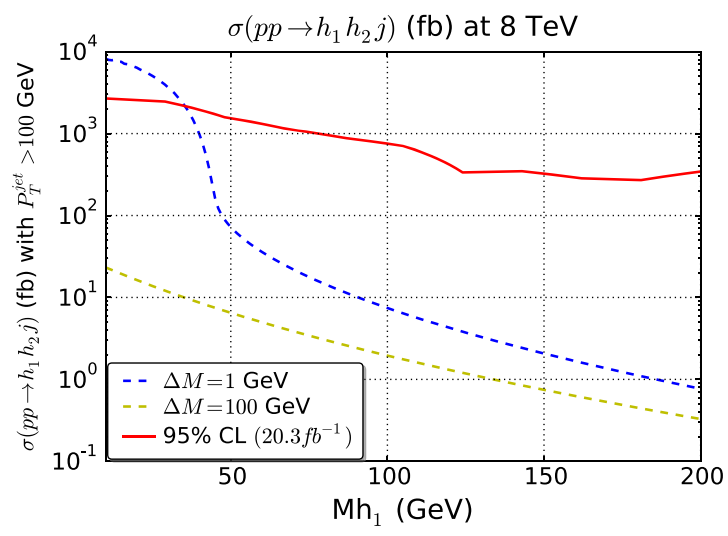

(a) $M_{h_{1}}$ vs cross section at $8 \mathrm{TeV}$.

\section{CONSTRAINING i2HDM: FUTURE PROJECTIONS}

Taking into consideration these collider limits and also adding the projections of the direct detection XENON1T experiment, we are able to impose the complete set of constraints on the i2HDM parameter space. It is worth stressing that, as before, we present the limits using the rescaled DD cross section $\hat{\sigma}_{\mathrm{SI}}=R_{\Omega} \times \sigma_{\mathrm{SI}}$, where $R_{\Omega}=\Omega_{\mathrm{DM}} / \Omega_{\mathrm{DM}}^{\text {Planck }}$, which allows us to take into account additional sources that could contribute to the DM relic density.

\section{A. Highlighting the "always allowed" regions}

The results of the constraints are presented in Fig. 22 as the color map of DM relic density in the $\left(M_{h_{1}}, \lambda_{345}\right)$ plane together with the projected sensitivity of the LHC@13 TeV with $3 \mathrm{ab}^{-1}$ of integrated luminosity using $h_{1} h_{2} j$ and $h_{1} h_{1} j$ channels, as well as a projection for the XENON1T experiment 95\% C.L. exclusion regions. These constraints are indicated by black, dark grey, and light grey colors, respectively. In this figure we plot excluded points on the top of the allowed points demonstrating the colored region of the parameter space which will always be allowed (AA). Figures 22(a) and 22(b) present AA parameter space for the combined constraints (black on the top of dark grey and dark grey on the top of light grey) for large and zoomed $\left(M_{h_{1}}, \lambda_{345}\right)$ regions, respectively, while Figs. 22(c) and 22(d) present AA regions for separate XENON1T and $h_{1} h_{1}+$ jet LHC13 constraints, respectively. From Fig. 22(a)-22(c) one can see how constraints from the LHC and XENON1T are complementary to each other. One can see that XENON1T will exclude large $M_{h_{1}}$ masses for

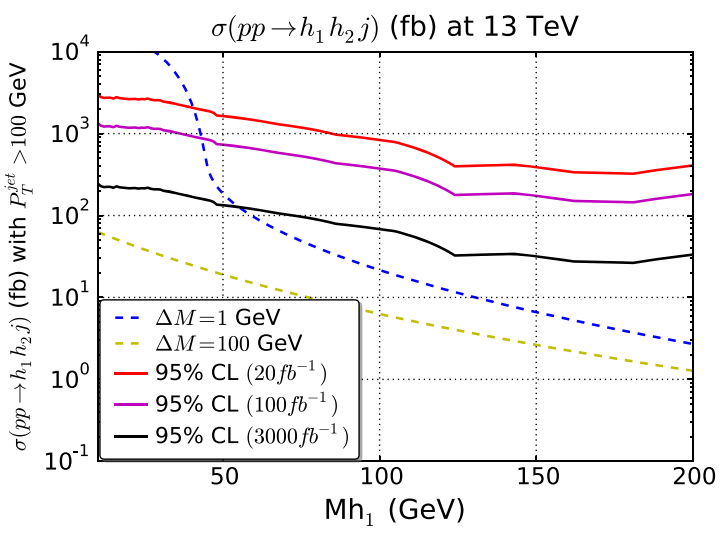

(b) $M_{h_{1}}$ vs cross section at $13 \mathrm{TeV}$.

FIG. 21. Cross sections and 95\% C.L.s for $p p \rightarrow h_{1} h_{2} j$ versus $M_{h_{1}}$ at 8 and $13 \mathrm{TeV}$. In both cases, the cross sections are shown for two different values of $\Delta M=M_{h_{2}}-M_{h_{1}}$ : (i) $\Delta M=1 \mathrm{GeV}$ (blue dashed cuve), and (ii) $\Delta M=100$ (yellow dashed curve). (a) Results for $8 \mathrm{TeV}$, with limits (solid red curve) calculated using the ATLAS analysis [106]. (b) Results for 13 TeV, with projected limits for the ATLAS analysis [106] with luminosities of $20 \mathrm{fb}^{-1}, 100 \mathrm{fb}^{-1}$, and $3000 \mathrm{fb}^{-1}$ (red, magenta, and black solid lines) at $13 \mathrm{TeV}$. 


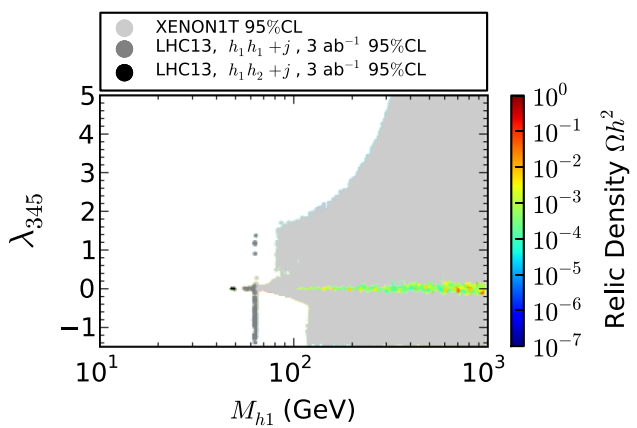

(a) Coloured points - always allowed points (AA)

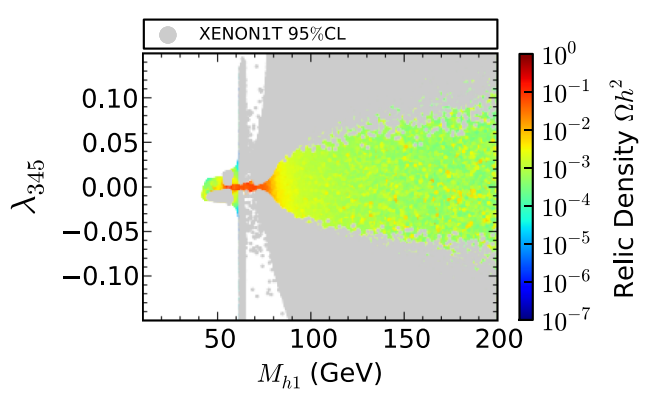

(c) Zoomed AA region only for XENON1T constraints

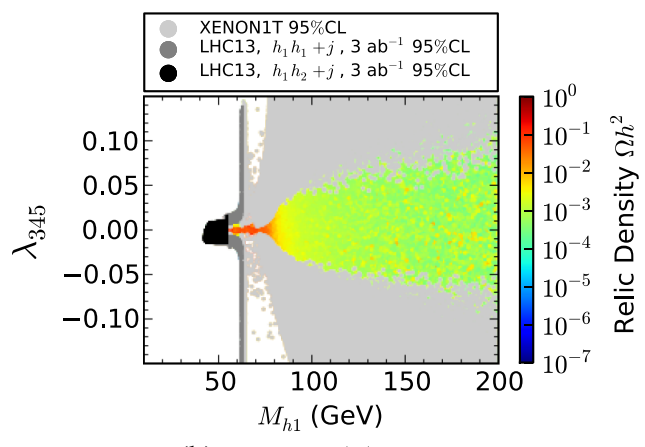

(b) Zoomed AA region

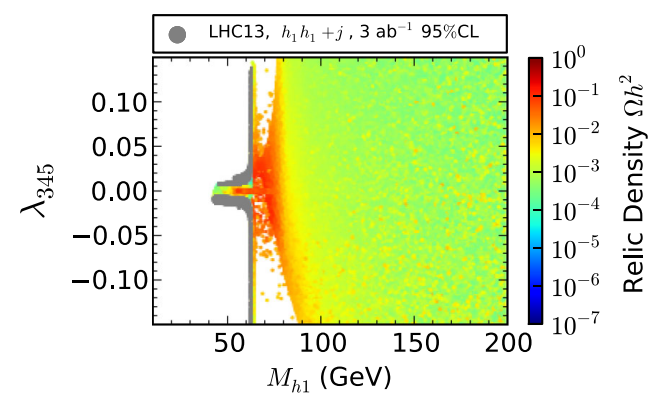

(d) Zoomed AA region only for the LHC13 constraints from $h 1 h 1+j$ signature

FIG. 22. Projection of the 5D random scan of the i2HDM into the $\left(M_{h_{1}}, \lambda_{345}\right)$ plane and the expected reach of the LHC@13 TeV with $3 \mathrm{ab}^{-1}$ of integrated luminosity using $h_{1} h_{2} j$ and $h_{1} h_{1} j$ channels as well as for XENON1T experiment indicated by black, dark grey, and light grey colors, respectively. Excluded points are plotted on the top of the allowed points demonstrating the colored region of the parameter space which will always be allowed (AA): (a) and (b) present AA parameter space for the combined constraints for large and zoomed $\left(M_{h_{1}}, \lambda_{345}\right)$ regions, respectively; (c) and (d) present AA regions for separate XENON1T and $h_{1} h_{1}+$ jet LHC13 constraints, respectively.

large enough values of $\lambda_{345}$ while the LHC will probe the region of smaller values of $\lambda_{345}$ for $M_{h_{1}}$ below the $M_{H} / 2$ threshold using the $h_{1} h_{1} j$ channel, and will cover all values of $\lambda_{345}$ using the $h_{1} h_{2} j$ channel for $M_{h_{1}}$ below $55 \mathrm{GeV}$.

\section{B. Highlighting the "always excluded" regions}

Besides the AA region it is informative to find and analyze the region with allowed points on the top of excluded points; therefore the black and grey colors present the region which always will be probed-and in the case of negative results, always excluded (AE) - by the above experiments. Such a region is presented in Figs. 23(a) and 23(b) in exact analogy to Figs. 22(a) and 22(b).

When comparing Figs. 22(a) and 22(b) and Figs. 23(a) and 23(b) - i.e. the plots with AA versus AE points-one observes a big difference between the order of the overlay of the excluded and allowed points. This is related to the fact that the $\Omega_{\mathrm{DM}} h^{2}$ can substantially vary: even for fixed $M_{h_{1}}$ and $\lambda_{345}$ values, a large $M_{h^{+}}$or $M_{h_{2}}$ can provide, respectively, large quartic couplings $h_{1} h_{1} W_{L} W_{L}$ and $h_{1} h_{1} Z_{L} Z_{L}$ [see Eq. (8)], which lead in their turn to an effective $h_{1} h_{1} \rightarrow V V$ annihilation. This brings the relic density down and avoids the XENON1T constraints (once we use DD rates rescaled to relic density). In the $\left(\lambda_{345}, M_{h_{1}}\right)$ plane, for example, these points overlap with the points where the quartic couplings mentioned above are small and the $\Omega_{\mathrm{DM}} h^{2}$ (and, respectively, exclusion) is driven only by $\lambda_{345}$. So the most complete picture comes from the combination of AA and AE plots: the most conservative allowed region comes from AA plots of Fig. 22, while the most conservative exclusion region is presented by $\mathrm{AE}$ plots of Fig. 23.

From Figs. 22 and 23 one can see that imposition of the XENON1T constraint reduces substantially the parameter space, greatly expanding the previous limits imposed by LUX. This effect is not so evident in the other planes, presented in Fig. 24 in analogy to Fig. 23, because the spinindependent cross section $\hat{\sigma}_{\mathrm{SI}}$ for DD is driven by the $t$-channel Higgs boson exchange and therefore is proportional to $\lambda_{345}^{2}$.

One should stress again the importance of the $p p \rightarrow$ $h_{1} h_{2}+j$ process, using which one can exclude $M_{h_{1}}<$ $55 \mathrm{GeV}$ when $M_{h_{2}}-M_{h_{1}}$ is small for all values of $\lambda_{345}$. This is shown clearly with the black dots in Figs. 24(b) and 24(d) where the (co)annihilation and respective mass 


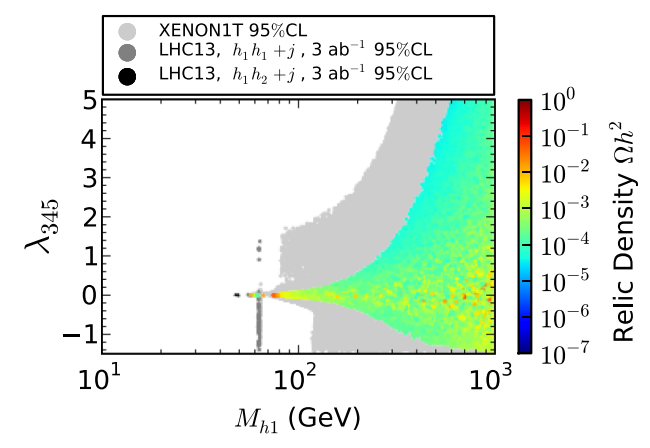

(a) Grey-black points - always excluded points (AE)

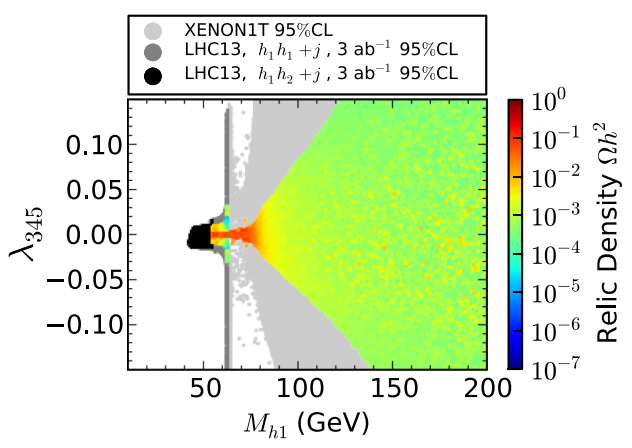

(b) Zoomed region with AE points

FIG. 23. Projection of the 5D random scan of the i2HDM into the $\left(M_{h_{1}}, \lambda_{345}\right)$ plane and the expected reach of the LHC@13 TeV with $3 \mathrm{ab}^{-1}$ of integrated luminosity using $h_{1} h_{2} j$ and $h_{1} h_{1} j$ channels as well as for the XENON1T experiment. Allowed points are on the top of the excluded ones; therefore the black and grey colors present the region which will always be excluded (AE) or probed by the above experiments.

degeneracy between $M_{h_{1}}$ and $M_{h_{2}}$ take place. Finally the $p p \rightarrow h_{1} h_{1}+j$ process imposes an extra constraint for the zone with low relic density corresponding to the $h_{1} h_{1} \rightarrow H$ resonant annihilation just above $M_{h_{1}}=M_{H} / 2$. In this case the invisible Higgs decay $H \rightarrow h_{1} h_{1}$ is closed and there is no restriction on $\left|\lambda_{345}\right|$, as we can see in Figs. 24(b) and 24(d) represented by the dark grey points.

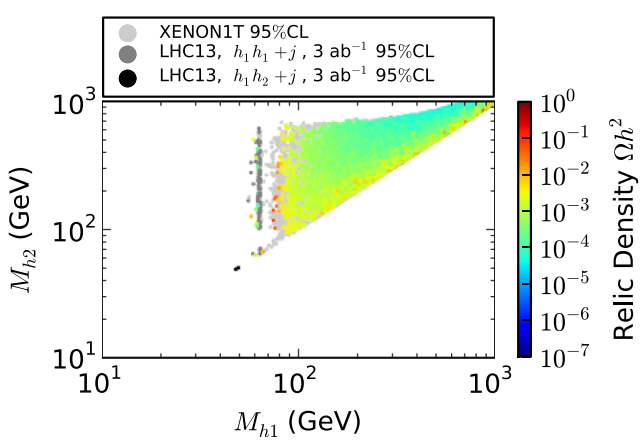

(a)

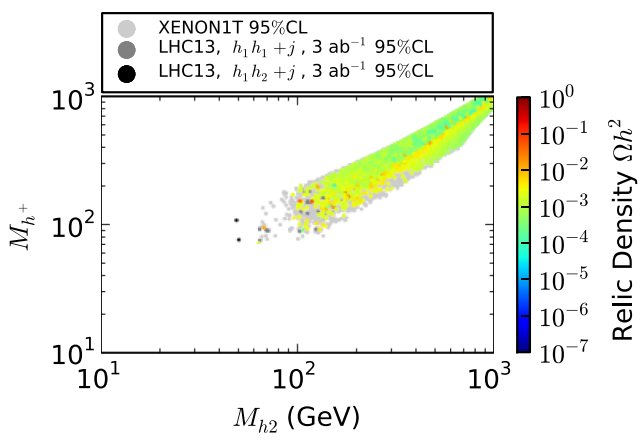

(c)

\section{Fitting the PLANCK data: Future projections}

We have also found the projected limits from colliders of monojet signatures and the XENON1T DD experiment for the i2HDM points which satisfy both the upper and the lower PLANCK limits, Eq. (30). In this case, the scattering cross section $\sigma_{\mathrm{SI}}$ is not rescaled, because we are in the zone with the right amount of DM relic density. The results of

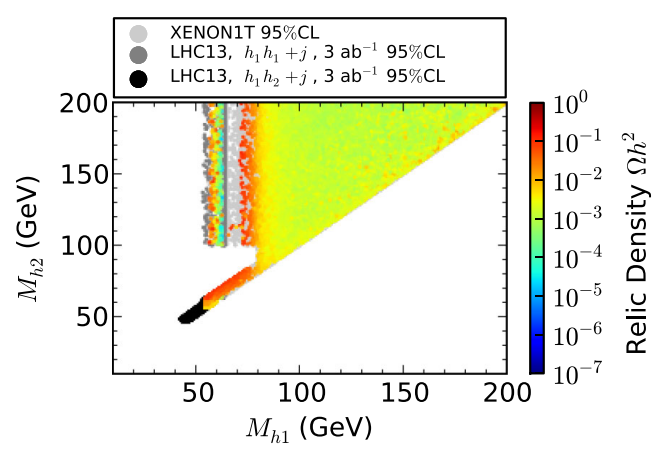

(b)

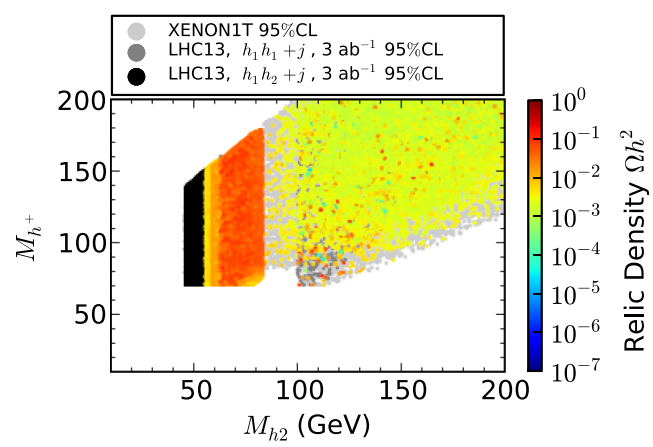

(d)

FIG. 24. Projection of the 5D random scan of the i2HDM into (a),(b) $\left(M_{h_{1}}, M_{h_{2}}\right)$ and (c),(d) $\left(M_{h_{2}}, M_{h^{+}}\right)$planes and the expected reach of the LHC@13 TeV with $3 \mathrm{ab}^{-1}$ of integrated luminosity using $h_{1} h_{2} j$ and $h_{1} h_{1} j$ channels as well as for the XENON1T experiment. Allowed points are on the top of the excluded ones, presenting AE points. The left panels (a),(c) present a bigger region of the parameter space, while the right ones (b),(d) present a zoomed region with AE points. 


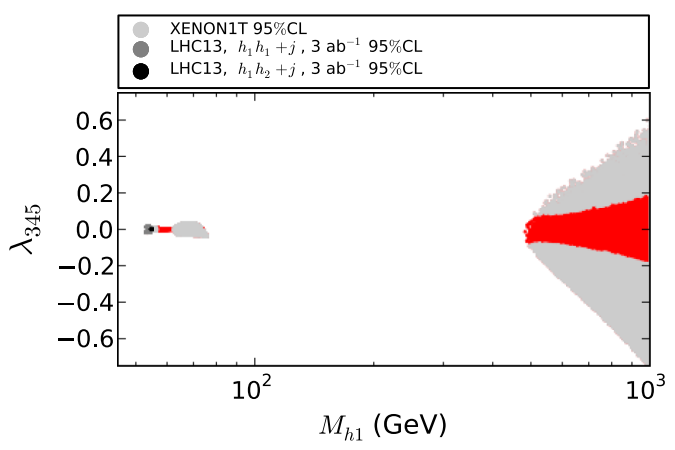

(a)

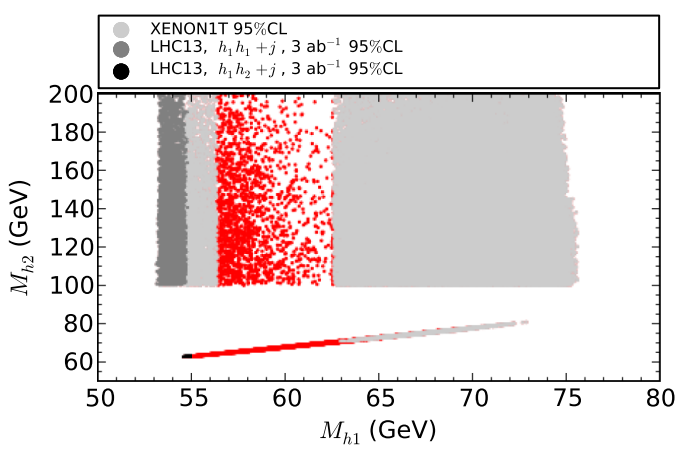

(c)

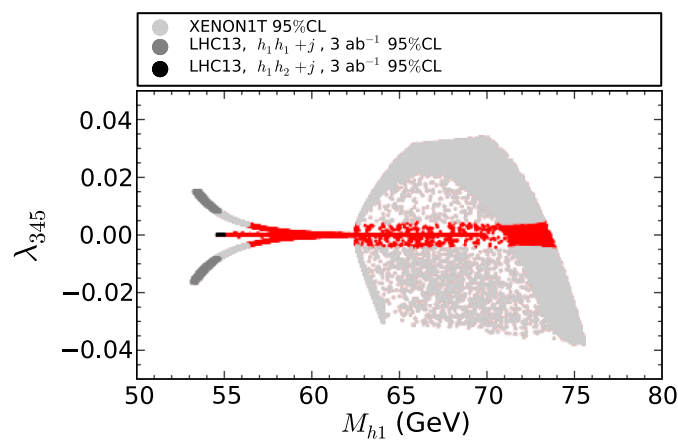

(b)

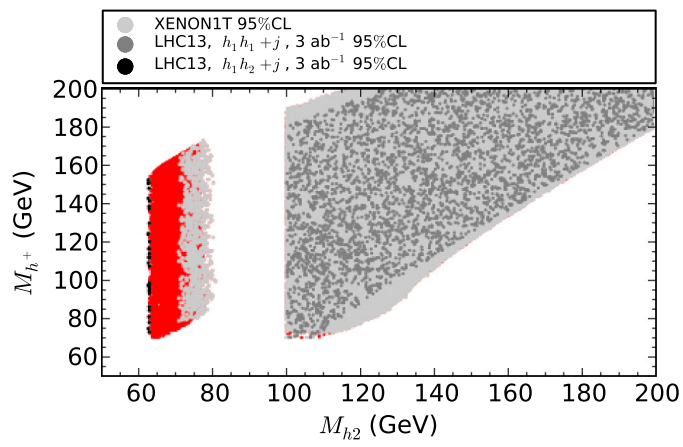

(d)

FIG. 25. The 2D projections of the 5D random scan of the i2HDM satisfying all constraints (Cut-1 to Cut-4) considered above for Figs. 26 and 27 plus the lower limit on the constraint on relic density given by Eq. (30), taking into consideration the collider limits of monojet signatures at $13 \mathrm{TeV}$ with $3 \mathrm{ab}^{-1}$ of integrated luminosity and the projections of the DD XENON1T experiment. In the first row we present the parameter space of the plane $\left(M_{h_{1}}, \lambda_{345}\right)$ in the range $\left.\subset[10 \mathrm{GeV}-1000 \mathrm{GeV}]\right)$. In the second row we present the planes $\left(M_{h_{1}}, \lambda_{345}\right)$ and $\left(M_{h_{1}}, M_{h_{2}}\right)$ in the range $\left.\subset[50 \mathrm{GeV}-80 \mathrm{GeV}]\right)$.

these constraints are presented in Fig. 25 as a scatter plot where the red zones represent the right amount of DM relic density. In the first row we show the parameter space of the plane $\left(M_{h_{1}}, \lambda_{345}\right)$ in the full mass range from 10 to $1000 \mathrm{GeV}$. In the second row we present the planes $\left(M_{h_{1}}, \lambda_{345}\right)$ and $\left(M_{h_{1}}, M_{h_{2}}\right)$ but in a narrow mass range between 50 and $80 \mathrm{GeV}$.

As we can see, the incorporation of the DD constraint sets important restrictions on the parameter space. Still, in Fig. 25(a) there is a zone in the upper mass range that is not ruled out. Also in the low mass range there is a region between $55 \mathrm{GeV}<M_{h_{1}}<74 \mathrm{GeV}$ which survives the restrictive constraint for small values of $\lambda_{345}$. We zoom into the surviving low mass region in Figs. 25(b) and 25(c). Because of the improved limits of the DD experiment, the parameter $\lambda_{345}$ is very sensitive to the scattering cross section, which sets a limit of $\left|\lambda_{345}\right|<0.01$ for this mass range.

The $p p \rightarrow h_{1} h_{2}+j$ process sets the exclusion limit for $M_{h_{1}}<55 \mathrm{GeV}$ (black dots) at the beginning of the $h_{1} h_{2}$ coannihilation region represented by the thin horizontal strip for very small values of $\lambda_{345}$ in Fig. 25(b), which is also seen in the lower part of Fig. 25(c). The $p p \rightarrow h_{1} h_{1}+j$ process imposes an extra constraint on the lower mass zone where the DM annihilates through the Higgs boson exchange and is visible in Fig. 25(b) in the shape of two symmetric wings for negative and positive values of $\lambda_{345}$. This excludes the $M_{h_{1}}<55 \mathrm{GeV}$ region. XENON1T will improve this constraint and exclude the $M_{h_{1}}<56.5 \mathrm{GeV}$ region.

\section{CONCLUDING REMARKS}

The i2HDM is a clear example of a minimal consistent DM model which is very well motivated by theoretical considerations. At the same time this model could provide monojet, mono- $Z$, mono-Higgs, and $\mathrm{VBF}+\boldsymbol{E}_{\mathrm{T}}^{\text {miss }}$ signatures at the LHC complemented by signals in direct and indirect DM search experiments.

The model is implemented into the CALCHEP and MICROMEGAs packages and is publicly available at the HEPMDB database together with the LanHEP model source. It is ready for further exploration in the context of the LHC, relic density, and DM direct detection.

In this paper we have performed detailed analysis of the constraints in the full 5D parameter space of the i2HDM from perturbativity, unitarity, electroweak precision data, Higgs data from the LHC, DM relic density, direct/indirect 
DM detection, and the LHC monojet analysis as well as implications of experimental LHC studies on disappearing charged tracks relevant to the high DM mass region. The LHC monojet analysis for the i2HDM model has been performed at the fast detector simulation level and provides new results together with limits from disappearing charged tracks at the LHC. Our results on non-LHC constraints are summarized in Figs. 6-8 in Sec. III as well as in more detailed Figs. 26-28 in Appendix which show the effect of consequent application of constraints from the following: (a) vacuum stability, perturbativity, and unitarity; (b) electroweak precision data, LEP constraints, and the LHC Higgs data; and (c) relic density constraints as well as constraints from LUX on DM from direct detection. In this paper we have explored for the first time the parameter space where DM from the i2HDM is underabundant implying an additional source of DM, using the above constraints complemented by the collider searches. We have also explored the parameter space in which the DM candidate of i2HDM represents $100 \%$ of the total DM budget of the Universe. We found that the parameter space with $M_{h_{1}}, M_{h_{2}}<45 \mathrm{GeV}$ or $M_{h^{+}}<70 \mathrm{GeV}$ is completely excluded, confirming the first limit found previously complemented the second one found in this study.

Though in general the parameter space of the i2HDM is five-dimensional, the parameter space relevant to the LHC monojet signature is only one or two dimensional, so the model can be easily explored at the LHC. There are two qualitatively different and complementary channels in monojet searches: $p p \rightarrow h_{1} h_{1} j$ and $p p \rightarrow h_{1} h_{2} j$, with the second one being relevant to the monojet signature when the mass gap between $h_{2}$ and $h_{1}$ is of the order of a few GeV. In the case of $h_{1} h_{2}$ degeneracy, the rate for $p p \rightarrow$ $h_{1} h_{1} j$ will be effectively doubled since $g_{H h_{2} h_{2}}=g_{H h_{1} h_{1}}$ [see Eq. (8)], and this easily can be taken into account for the estimation of constraints in the respective region of the parameter space. For a fixed $M_{h_{1}}$, the strength of the first process depends only on $\lambda_{345}$ because the Higgs boson is the only mediator, while the strength of the second process is fixed by the weak coupling since the $Z$ boson is the only mediator for this process. The last process is important to cover the $h_{1} h_{2}$ coannihilation region available for $54 \mathrm{GeV}<M_{h_{1}}<73 \mathrm{GeV}$, where the relic density agrees with the PLANCK data. The results on this process and on this region are new to our best knowledge. Therefore these two processes complement each other in covering the parameter space: for large values of $\lambda_{345}, p p \rightarrow h_{1} h_{1} j$ would be the dominant LHC signature, while for small or vanishing values of $\lambda_{345}$, the $p p \rightarrow h_{1} h_{2} j$ process will cover additional parameter space as demonstrated in Figs. 22-25.

Talking about quantitative results, the LHC has rather limited potential to probe $M_{h_{1}}$ with the monojet signature. Even for the projected luminosity of $3 \mathrm{ab}^{-1}$, we have found that the LHC could set a limit on $M_{h_{1}}$ up to $83 \mathrm{GeV}$ from the $p p \rightarrow h_{1} h_{1} j$ process with the maximal value allowed for $\lambda_{345}$ and only up to $55 \mathrm{GeV}$ from $p p \rightarrow h_{1} h_{2} j$ for any value of $\lambda_{345}$, covering just the tip of the $h_{1} h_{2}$ coannihilation region. Such a weak sensitivity of the LHC is related to the similarity between the shapes of the $\boldsymbol{E}_{\mathrm{T}}^{\text {miss }}$ distribution of the dominant $Z j \rightarrow \nu \nu j$ background and that of the signal which has the same $Z$-boson mediator, while the DM mass is not very different from $M_{Z} / 2$, which as shown in [15] is the reason for such a similarity in $\boldsymbol{E}_{\mathrm{T}}^{\text {miss }}$ shape. At the same time, the potential of the LHC using a search for disappearing charged tracks is quite impressive in probing $M_{h_{1}}$ masses up to about $500 \mathrm{GeV}$ already at $8 \mathrm{TeV}$ with $19.5 \mathrm{fb}^{-1}$ luminosity as we have found in our study.

We have also explored the projected potential of XENON1T to probe the i2HDM parameter space and have found that it is quite impressive, confirming results of previous studies. In our study we have presented "absolutely allowed" and "absolutely excluded" points in different projections of the i2HDM 5D space demonstrating different features of the models and the potential of current and future experiments. In general, DM DD experiments and collider searches complement each other: the $p p \rightarrow$ $h_{1} h_{1} j$ process covers in the region with large $\lambda_{345}$ coupling where DM DD rates are low because of the low relic density rescaling, while the $p p \rightarrow h_{1} h_{2} j$ process is sensitive to the parameter space with low $\lambda_{345}$ where DM DD rates are low because of the low rate of DM scattering off the nuclei.

\section{ACKNOWLEDGMENTS}

A. B. thanks Tania Robens and Alexander Pukhov for useful discussions. A. B. and M. T. acknowledge partial support from the STFC Grant No. ST/L000296/1 and the NExT Institute, FAPESP Grant No. 2011/11973-4 for funding their visit to ICTP-SAIFR as well as SOTONFAPESP collaboration grant. A.B. thanks the Royal Society Leverhulme Trust Senior Research Fellowship No. LT140094. M. T. acknowledges support from an STFC STEP award. G. C. acknowledges partial support from the Labex-LIO (Lyon Institute of Origins) under Grant No. ANR-10-LABX-66 and FRAMA (FR3127, Fédération de Recherche "André Marie Ampère"). I. P. I. acknowledges funding from Fundação para a Ciência e a Tecnologia (FCT) through the Investigator Contract No. IF/ 00989/2014/CP1214/CT0004 under the IF2014 Program and through FCT Contracts No. UID/FIS/00777/2013 and No. CERN/FIS-NUC/0010/2015, which are partially funded through POCTI, COMPETE, QREN, and the European Union.

\section{APPENDIX: NUMERICAL SCAN: DETAILED DISCUSSION}

To have a complete picture of the properties of i2HDM in the whole parameter space, we have performed 
a five-dimensional random scan of the model parameter space with about $10^{8}$ points, evaluating all relevant observables and limits mentioned above. The range for the model parameters of the scan was chosen according to Eq. (18).

To better delineate the impact of each constraint, we have imposed different cuts on the parameter space sequentially, following the classification below:

Cut-1: theoretical constraints on the potential from vacuum stability [Eqs. (11), (12), and (17)], perturbativity and unitarity [Eqs. (13)-(16)];

Cut-2: constraints from LEP [Eqs. (19) and (23)], EWPT [Eq. (22)], and the LHC Higgs data [Eqs. (25) and (26)];

Cut-3: constraint on the relic density $\left[\Omega_{\mathrm{DM}} h^{2} \leq 0.1184+\right.$ $2 \times 0.012]$, where we consider only the upper bound within 2 standard deviations;
Cut-4: constraints from DM DD searches from LUX.

The results of the scan are presented in Fig. 26 in the form of a color map of DM relic density, projected on twodimensional planes: $\left(M_{h_{1}}, \lambda_{345}\right)$ in the first, $\left(M_{h_{1}}, M_{h_{2}}\right)$ in the second, and $\left(M_{h_{2}}, M_{h^{+}}\right)$in the third column, respectively. The four rows reproduce the effect of the progressive application of the four cuts defined above. In Fig. 27 we also present, in the same format, the results of a finer scan, zoomed to the region of low masses, where the range has been restricted to $10-200 \mathrm{GeV}$ for the three masses $M_{h_{1}}, M_{h_{2}}$, and $M_{h^{+}}$. The latter is the most relevant corner of parameter space for the LHC phenomenology that we will discuss in the next section. Note that the lower bound of $\lambda_{345}$ presented in these plots corresponds to the lowest limits allowed by unitarity, perturbativity, and scalar potential constraints (see Fig. 2).

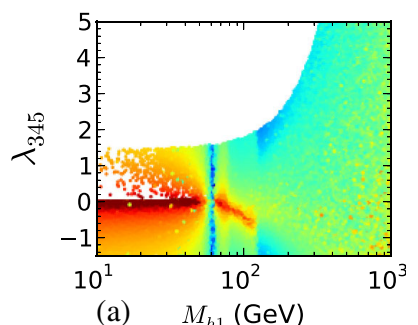

(a) $\quad M_{h 1}(\mathrm{GeV})$

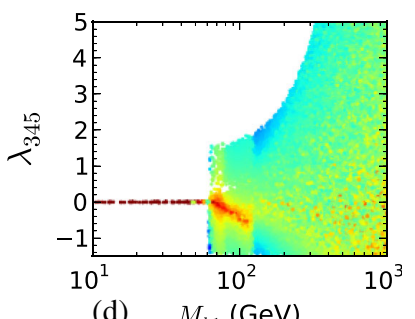

(d) $\quad M_{h 1}(\mathrm{GeV})$
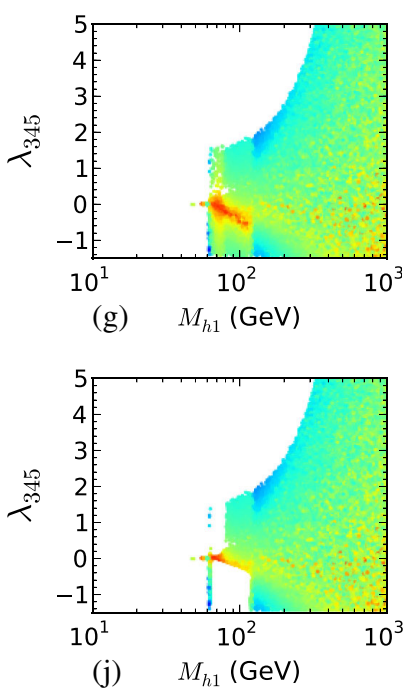

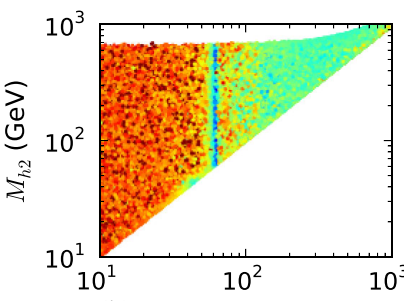

(b) $\quad M_{h 1}(\mathrm{GeV})$

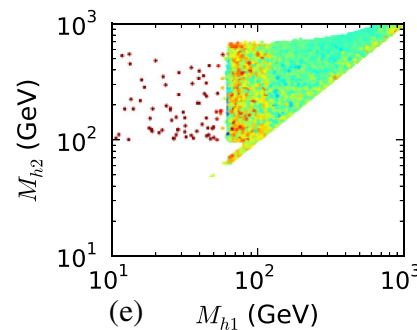

(e) $\quad M_{h 1}(\mathrm{GeV})$
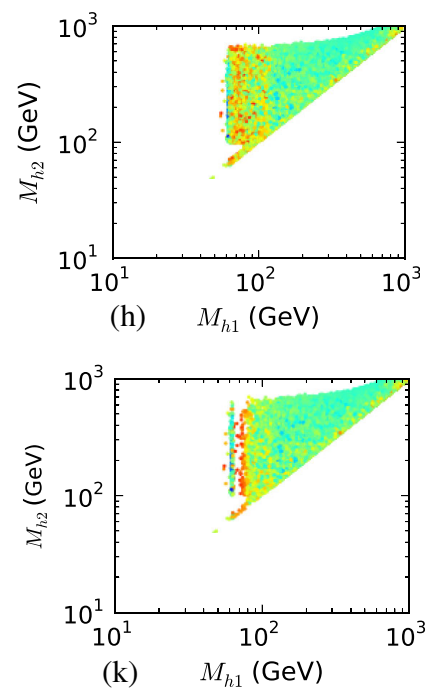

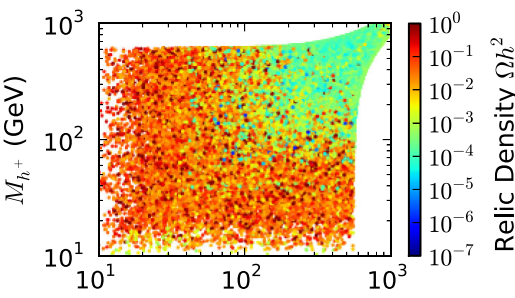

(c) $\quad M_{h 2}(\mathrm{GeV})$
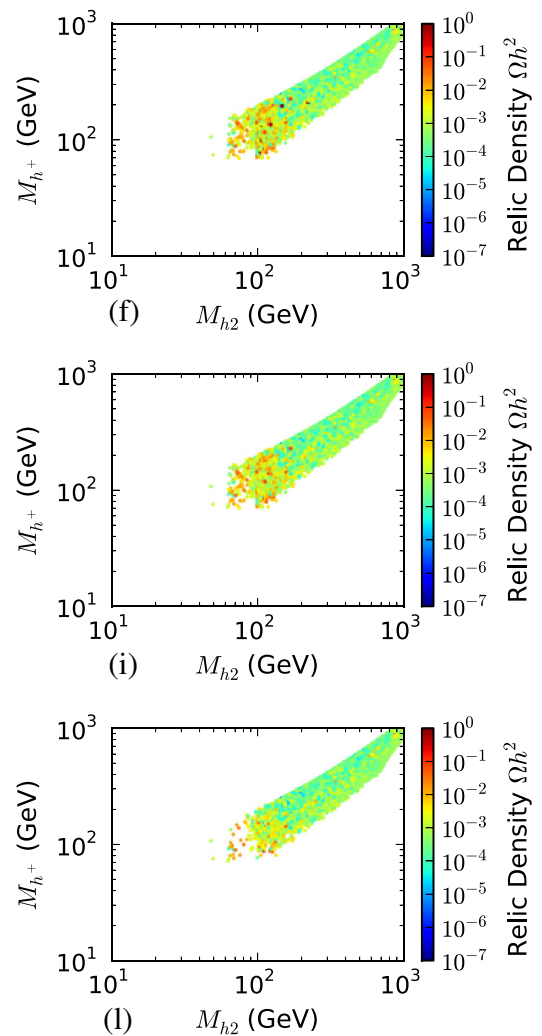

FIG. 26. Color maps of DM relic density for 2D projections of the 5D random scan of the i2HDM: each row demonstrates the effect of consequent application of the experimental and theoretical constraints in the $\left(M_{h_{1}}, \lambda_{345}\right),\left(M_{h_{1}}, M_{h_{2}}\right)$, and $\left(M_{h_{1}}, M_{h^{+}}\right)$planes. Each row corresponds to Cut-1-Cut-4, described in the text: Cut-1 for (a)-(c) [Eqs. (11)-(15)]; Cut-2 for (d)-(f) [Eqs. (19), (23), (22), (25), (26)]; Cut-3 for (g)-(i) $\left[\Omega_{\mathrm{DM}}^{\text {Planck }} h^{2} \leq 0.1184+2 \times 0.012\right]$; Cut-4 for (j)-(l) [LUX]. 

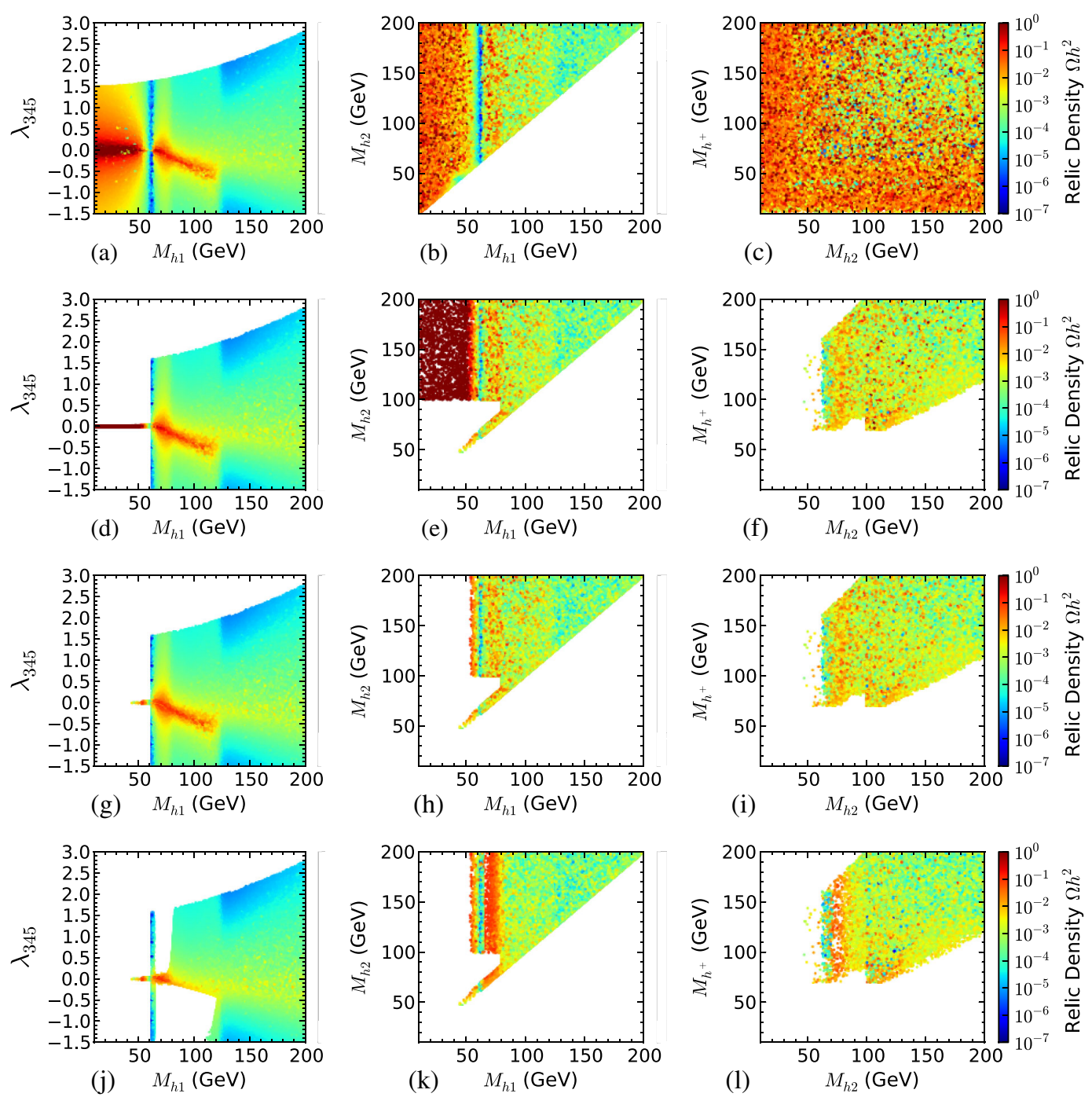

FIG. 27. Color maps of DM relic density for 2D projections of the 5D random scan of the i2HDM for the parameter space restricted to (10 GeV-200 GeV) for $M_{h_{1}}, M_{h_{2}}$, and $M_{h^{+}}$. As for Fig. 26 each row corresponds to Cut-1-Cut-4, described in the text: Cut-1 for (a)-(c) [Eqs. (11)-(15)]; Cut-2 for (d)-(f) [Eqs. (19), (23), (22), (25), (26)]; Cut-3 for (g)-(i) $\left[\Omega_{\mathrm{DM}}^{\text {Planck }} h^{2} \leq 0.1184+2 \times 0.012\right.$ ]; Cut-4 for (j)-(1) [LUX].

One can see from Figs. 26 and 27(a) that $\lambda_{345}$ is limited from above, and the dependence which defines the shape of this limit as a function of $M_{h_{1}}$ comes from the vacuum stability condition given by Eq. (16). One can also see from Figs. 26, 27(a), 27(b), and analogous figures in the rows below, that the relic density is too high for small $M_{h_{1}}$ values and small $\lambda_{345}$. Therefore, the relic density constraint combined with the LHC Higgs data constraints (limiting the invisible decays of the Higgs) restricts $M_{h_{1}}$ to be above $45 \mathrm{GeV}$, as it can clearly be seen from Figs. 26, 27(g), and 27(h). For example, the range $45 \mathrm{GeV}<M_{h_{1}}<50 \mathrm{GeV}$ is allowed but it requires $h_{1} h_{2}$ coannihilation and respective mass degeneracy, as one can see from Figs. 26, 27(h), and 27(k). From Figs. 26, 27(a), 27(b), and analogous ones in the rows below, one can see a clear vertical blue pattern of low relic density corresponding to the $h_{1} h_{1} \rightarrow H$ resonant annihilation. For $M_{h_{1}}>M_{H} / 2$ the pattern of DM relic density follows the pattern of $W W, Z Z$, and $H H$ thresholds presented earlier in Fig. 4.

One can also observe that the effect of Cut-1 plus Cut-2 is quite dramatic: (a) $\operatorname{Br}\left(H \rightarrow h_{1} h_{1}\right)<0.28$ and $\mu^{\gamma \gamma}=$ $1.14_{-0.36}^{+0.38}$ constraints require $\lambda_{345} \leq 0.02$ for $M_{h_{1}}<M_{H} / 2$ [Figs. 26-27(d)]; (b) LEP constraints require $M_{h_{2}} \gtrsim$ $100 \mathrm{GeV}$ if $M_{h_{2}}-M_{h_{1}}>8 \mathrm{GeV}$ [Figs. 26-27(e)]; and (c) LEP and LHC Higgs data constraints require $M_{h^{+}}>70 \mathrm{GeV}$, while $M_{h_{2}}$ is generically excluded below $M_{Z} / 2$ [Figs. 26-27(f)]. The effect from adding the (upper) cut from relic density (Cut-3) is shown in Figs. 26-27(g)27(i): one can see that this cut (combined with the previous ones) excludes $M_{h_{1}}<M_{Z} / 2$ for the whole i2HDM 


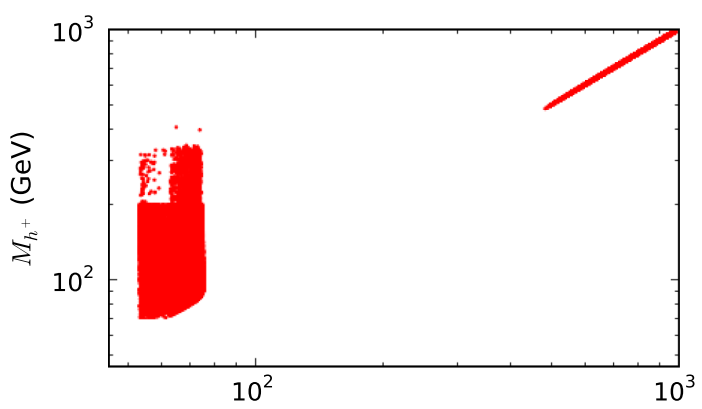

(a)

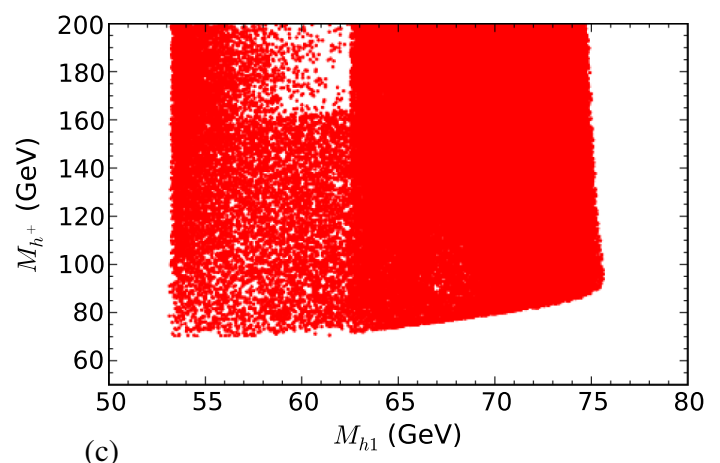

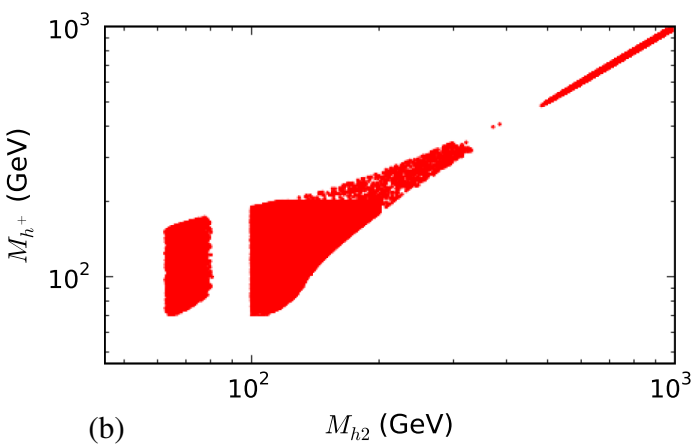

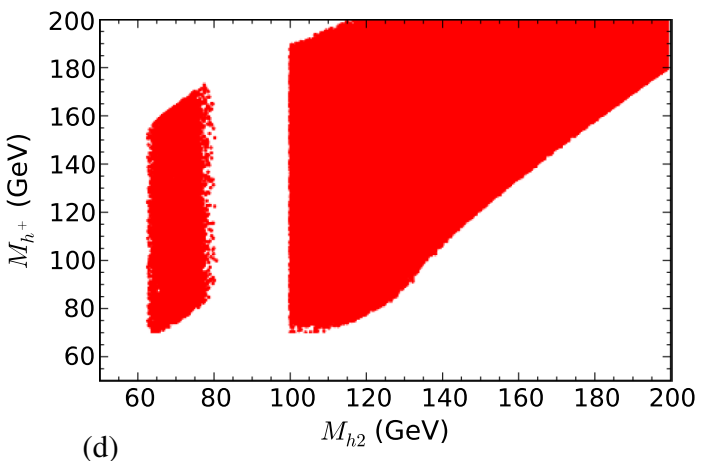

(d)

FIG. 28. The 2D projections of the random scan of the i2HDM for points satisfying all constraints and providing the correct relic abundance within two sigmas of the PLANCK result. The top row corresponds to the "full" parameter space $10 \mathrm{GeV}<M_{h_{1}}, M_{h_{2}}$, $M_{h^{+}}<1000 \mathrm{GeV}$, while the lower row refers to the "zoomed" parameter space $10 \mathrm{GeV}<M_{h_{1}}, M_{h_{2}}, M_{h^{+}}<200 \mathrm{GeV}$.

parameter space [Figs. 26-27(g) and 27(h)], but does not have a visible effect in the $\left(M_{h_{2}}, M_{h^{+}}\right)$plane [Figs. 26-27(i)]. Actually the region with $M_{h_{1}}<M_{Z} / 2$ is excluded due to the interplay of several constraints. In the $M_{h_{1}}<M_{H} / 2$ region with $\left|\lambda_{345}\right| \lesssim 0.02$ as required by LHC Higgs data, the only possibility for relic density of $h_{1}$ to be sufficiently low to satisfy the PLANCK constraints is the $h_{1} h_{2}$ coannihilation channel: potentially this coannihilation could provide low enough relic density for $M_{h_{1}}$ down to about $20 \mathrm{GeV}$. However, for $M_{h_{1}}+M_{h_{2}}<M_{Z}$ the $Z \rightarrow$ $h_{1} h_{2}$ decay is open and contributes significantly to the invisible Z-boson decay that is strongly limited by LEP. As the $Z$-boson partial width for this decay channel is defined just by $M_{h_{1}}$ and $M_{h_{2}}$, since $Z h_{1} h_{2}$ coupling is fixed by the gauge invariance, the $M_{h_{1}}+M_{h_{2}}<M_{Z}$ parameter space is completely excluded. For the $h_{1} h_{2}$ coannihilation region, this exclusion is equivalent to $M_{h_{1}}, M_{h_{2}} \gtrsim M_{Z} / 2$. The $h_{1} h_{2}$ coannihilation corridor which provides relic density below or equal to the PLANCK limit is clearly visible in Figs. 26-27(e), 27(h), 27(k).

The additional constraint from DM DD from LUX (Cut-4) removes a substantial portion of the parameter space for large and intermediate $\left|\lambda_{345}\right|$ values for $M_{h_{1}} \lesssim M_{H}$ [Figs. 26-27(j)]. In this excluded parameter space the scattering cross section of $h_{1}$ on the proton is quite large due to the Higgs boson exchange enhanced by $\left|\lambda_{345}\right|$, while the relic density is respectively low, again due to the large value $\left|\lambda_{345}\right|$, but it is not low enough to suppress the DM detection rate below the experimental exclusion. So, the LUX cut removes the low relic density region, and one can see this clearly in Figs. 26-27(k), 27(1) by the enhanced yellow-red color in the $M_{h_{1}} \lesssim M_{H}$ region in comparison to the respective Figs. 26-27(h), 27(i) where the DM DD cut was not applied. For $\lambda_{345} \gtrsim 0.2$ the parameter space is excluded for $M_{H} / 2<M_{h_{1}}<M_{W}$ while for $\lambda_{345} \lesssim-0.2$ it is excluded for $M_{H} / 2<M_{h_{1}}<M_{H}$ as illustrated in Figs. 26-27(j). Once the $h_{1} h_{1} \rightarrow W^{+} W^{-}$channel is open for positive $\lambda_{345}$, or the $h_{1} h_{1} \rightarrow H H$ channel is open for negative $\lambda_{345}$, the relic density drops substantially below the PLANCK limit, which makes the rescaling factor low enough to avoid limits from LUX searches. The difference between the positive and negative $\lambda_{345}$ cases is related to the respective positive and negative interference of the $h_{1} h_{1} \rightarrow$ $H \rightarrow X X$ channel with non-Higgs-exchange diagrams. This asymmetry between positive and negative $\lambda_{345}$ cases was seen initially in Fig. 4 , where the $h_{1}$ relic density was presented as a function of $M_{h_{1}}$ for different $\lambda_{345}$ values.

We would also like to point to some features of the scan for the region of $M_{h_{1}}, M_{h_{2}}$, and $M_{h^{+}}$above $200 \mathrm{GeV}$, presented in Fig. 26. From Figs. 26(f), 26(i), 26(1), one can see that EWPT constraints require a very modest mass split between $M_{h_{2}}$ and $M_{h^{+}}$since this mass split is directly related to values of the $M_{h_{2}}$ and $M_{h^{+}}$couplings to the SM Higgs as well as to the couplings to longitudinal 
components of the $W$ and $Z$ bosons. Therefore constraints from $S$ and $T$ parameters leave only a rather narrow corridor in the $\left(M_{h^{+}}, M_{h_{2}}\right)$ plane.

Finally, for the case when the relic density is required to fit the PLANCK result within two sigmas, 2D projections on the $\left(M_{h_{1}}, M_{h^{+}}\right)$and $\left(M_{h_{2}}, M_{h^{+}}\right)$are shown in Fig. 28.
The plots in the top row show results for the "full" scan $10 \mathrm{GeV}<M_{h_{1}}, M_{h_{2}}, M_{h^{+}}<1000 \mathrm{GeV}$, while in the lower row we present the "zoomed" scan $10 \mathrm{GeV}<$ $M_{h_{1}}, M_{h_{2}}, M_{h^{+}}<200 \mathrm{GeV}$. These plots complement the information on the surviving regions given by Fig. 8 in the main text.
[1] S. Chatrchyan et al. (CMS Collaboration), Observation of a new boson at a mass of $125 \mathrm{GeV}$ with the CMS experiment at the LHC, Phys. Lett. B 716, 30 (2012).

[2] G. Aad et al. (ATLAS Collaboration), Observation of a new particle in the search for the Standard Model Higgs boson with the ATLAS detector at the LHC, Phys. Lett. B 716, 1 (2012).

[3] P. J. Fox, R. Harnik, J. Kopp, and Y. Tsai, Missing energy signatures of dark matter at the LHC, Phys. Rev. D 85, 056011 (2012).

[4] A. Rajaraman, W. Shepherd, T. M. P. Tait, and A. M. Wijangco, LHC bounds on interactions of dark matter, Phys. Rev. D 84, 095013 (2011).

[5] J. Goodman, M. Ibe, A. Rajaraman, W. Shepherd, T. M. P. Tait, and H.-B. Yu, Constraints on dark matter from colliders, Phys. Rev. D 82, 116010 (2010).

[6] Y. Bai, P. J. Fox, and R. Harnik, The tevatron at the frontier of dark matter direct detection, J. High Energy Phys. 12 (2010) 048.

[7] M. Beltran, D. Hooper, E. W. Kolb, Z. A. C. Krusberg, and T. M.P. Tait, Maverick dark matter at colliders, J. High Energy Phys. 09 (2010) 037.

[8] J. Goodman, M. Ibe, A. Rajaraman, W. Shepherd, T. M. P. Tait, and H.-B. Yu, Constraints on light Majorana dark matter from colliders, Phys. Lett. B 695, 185 (2011).

[9] P. J. Fox, R. Harnik, J. Kopp, and Y. Tsai, LEP shines light on dark matter, Phys. Rev. D 84, 014028 (2011).

[10] I. M. Shoemaker and L. Vecchi, Unitarity and monojet bounds on models for DAMA, CoGeNT, and CRESST-II, Phys. Rev. D 86, 015023 (2012).

[11] P. J. Fox and C. Williams, Next-to-leading order predictions for dark matter production at hadron colliders, Phys. Rev. D 87, 054030 (2013).

[12] U. Haisch, F. Kahlhoefer, and J. Unwin, The impact of heavy-quark loops on LHC dark matter searches, J. High Energy Phys. 07 (2013) 125.

[13] G. Busoni, A. De Simone, E. Morgante, and A. Riotto, On the validity of the effective field theory for dark matter searches at the LHC, Phys. Lett. B 728, 412 (2014).

[14] G. Busoni, A. De Simone, J. Gramling, E. Morgante, and A. Riotto, On the validity of the effective field theory for dark matter searches at the LHC. Part II: Complete analysis for the $s$-channel, J. Cosmol. Astropart. Phys. 06 (2014) 060.

[15] A. Belyaev, L. Panizzi, A. Pukhov, and M. Thomas, Dark matter characterization at the LHC in the effective field theory approach, J. High Energy Phys. 04 (2017) 110.
[16] O. Buchmueller, M. J. Dolan, and C. McCabe, Beyond effective field theory for dark matter searches at the LHC, J. High Energy Phys. 01 (2014) 025.

[17] G. Busoni, A. De Simone, J. Gramling, E. Morgante, and A. Riotto, On the validity of the effective field theory for dark matter searches at the LHC. Part II: Complete analysis for the $s$-channel, J. Cosmol. Astropart. Phys. 06 (2014) 060 .

[18] G. Busoni, A. De Simone, T. Jacques, E. Morgante, and A. Riotto, On the validity of the effective field theory for dark matter searches at the LHC. Part III: Analysis for the $t$-channel, J. Cosmol. Astropart. Phys. 09 (2014) 022.

[19] O. Buchmueller, M. J. Dolan, S. A. Malik, and C. McCabe, Characterising dark matter searches at colliders and direct detection experiments: Vector mediators, J. High Energy Phys. 01 (2015) 037.

[20] M. R. Buckley, D. Feld, and D. Goncalves, Scalar simplified models for dark matter, Phys. Rev. D 91, 015017 (2015).

[21] J. Abdallah et al., Simplified models for dark matter searches at the LHC, Phys. Dark Universe 9, 8 (2015).

[22] J. Abdallah et al., Simplified models for dark matter and missing energy searches at the LHC, arXiv:1409.2893.

[23] D. Abercrombie et al., Dark matter benchmark models for early LHC Run-2 searches: Report of the ATLAS/CMS Dark Matter Forum, arXiv:1507.00966.

[24] N. G. Deshpande and E. Ma, Pattern of symmetry breaking with two Higgs doublets, Phys. Rev. D 18, 2574 (1978).

[25] E. Ma, Verifiable radiative seesaw mechanism of neutrino mass and dark matter, Phys. Rev. D 73, 077301 (2006).

[26] R. Barbieri, L. J. Hall, and V. S. Rychkov, Improved naturalness with a heavy Higgs: An alternative road to LHC physics, Phys. Rev. D 74, 015007 (2006).

[27] L. Lopez Honorez, E. Nezri, J. F. Oliver, and M. H. Tytgat, The inert doublet model: An archetype for dark matter, J. Cosmol. Astropart. Phys. 02 (2007) 028.

[28] M. Krawczyk, D. Sokolowska, P. Swaczyna, and B. Swiezewska, Constraining inert dark matter by $R_{\gamma \gamma}$ and WMAP data, J. High Energy Phys. 09 (2013) 055.

[29] A. Ilnicka, M. Krawczyk, and T. Robens, Inert doublet model in light of LHC Run I and astrophysical data, Phys. Rev. D 93, 055026 (2016).

[30] M. A. Díaz, B. Koch, and S. Urrutia-Quiroga, Constraints to dark matter from inert Higgs doublet model, Adv. High Energy Phys. 16, 8278375 (2016).

[31] N. Chakrabarty, D. K. Ghosh, B. Mukhopadhyaya, and I. Saha, Dark matter, neutrino masses and high scale validity 
of an inert Higgs doublet model, Phys. Rev. D 92, 015002 (2015).

[32] N. Khan and S. Rakshit, Constraints on inert dark matter from the metastability of the electroweak vacuum, Phys. Rev. D 92, 055006 (2015).

[33] J.-O. Gong, H. M. Lee, and S. K. Kang, Inflation and dark matter in two Higgs doublet models, J. High Energy Phys. 04 (2012) 128.

[34] C. Arina, F.-S. Ling, and M. H. G. Tytgat, IDM and iDM or the inert doublet model and inelastic dark matter, J. Cosmol. Astropart. Phys. 10 (2009) 018.

[35] K. P. Modak and D. Majumdar, Confronting galactic and extragalactic $\gamma$-rays observed by Fermi-lat with annihilating dark matter in an inert Higgs doublet model, Astrophys. J. Suppl. Ser. 219, 37 (2015).

[36] F. S. Queiroz and C. E. Yaguna, The CTA aims at the inert doublet model, J. Cosmol. Astropart. Phys. 02 (2016) 038.

[37] C. Garcia-Cely, M. Gustafsson, and A. Ibarra, Probing the inert doublet dark matter model with Cherenkov telescopes, J. Cosmol. Astropart. Phys. 02 (2016) 043.

[38] P. Agrawal, E. M. Dolle, and C. A. Krenke, Signals of inert doublet dark matter in neutrino telescopes, Phys. Rev. D 79, 015015 (2009).

[39] S. Andreas, M. H. G. Tytgat, and Q. Swillens, Neutrinos from inert doublet dark matter, J. Cosmol. Astropart. Phys. 04 (2009) 004.

[40] E. Nezri, M. H. G. Tytgat, and G. Vertongen, $e+$ and anti- $p$ from inert doublet model dark matter, J. Cosmol. Astropart. Phys. 04 (2009) 014.

[41] N. Turok and J. Zadrozny, Phase transitions in the two doublet model, Nucl. Phys. B369, 729 (1992).

[42] W. N. Cottingham and N. Hasan, Two Higgs doublet potential at finite temperature, Phys. Rev. D 51, 866 (1995).

[43] I. F. Ginzburg, I. P. Ivanov, and K. A. Kanishev, The evolution of vacuum states and phase transitions in 2HDM during cooling of Universe, Phys. Rev. D 81, 085031 (2010).

[44] I. F. Ginzburg, K. A. Kanishev, M. Krawczyk, and D. Sokolowska, Evolution of Universe to the present inert phase, Phys. Rev. D 82, 123533 (2010).

[45] T. A. Chowdhury, M. Nemevsek, G. Senjanovic, and Y. Zhang, Dark matter as the trigger of strong electroweak phase transition, J. Cosmol. Astropart. Phys. 02 (2012) 029.

[46] D. Borah and J. M. Cline, Inert doublet dark matter with strong electroweak phase transition, Phys. Rev. D 86, 055001 (2012).

[47] G. Gil, P. Chankowski, and M. Krawczyk, Inert dark matter and strong electroweak phase transition, Phys. Lett. B 717, 396 (2012).

[48] G. C. Dorsch, S. J. Huber, and J. M. No, A strong electroweak phase transition in the 2HDM after LHC8, J. High Energy Phys. 10 (2013) 029.

[49] J. M. Cline and K. Kainulainen, Improved electroweak phase transition with subdominant inert doublet dark matter, Phys. Rev. D 87, 071701 (2013).

[50] N. Blinov, S. Profumo, and T. Stefaniak, The electroweak phase transition in the inert doublet model, J. Cosmol. Astropart. Phys. 07 (2015) 028.
[51] A. Arhrib, R. Benbrik, and N. Gaur, $H \rightarrow \gamma \gamma$ in inert Higgs doublet model, Phys. Rev. D 85, 095021 (2012).

[52] B. Swiezewska and M. Krawczyk, Diphoton rate in the inert doublet model with a $125 \mathrm{GeV}$ Higgs boson, Phys. Rev. D 88, 035019 (2013).

[53] M. Krawczyk, D. Sokołowska, P. Swaczyna, and B. Świeżewska, Higgs $\rightarrow \gamma \gamma, Z \gamma$ in the inert doublet model, Acta Phys. Pol. B 44, 2163 (2013).

[54] X. Miao, S. Su, and B. Thomas, Trilepton signals in the inert doublet model, Phys. Rev. D 82, 035009 (2010).

[55] M. Gustafsson, S. Rydbeck, L. Lopez-Honorez, and E. Lundstrom, Status of the inert doublet model and the role of multileptons at the LHC, Phys. Rev. D 86, 075019 (2012).

[56] M. Hashemi and S. Najjari, Observability of inert scalars at the LHC, Eur. Phys. J. C 77, 592 (2017).

[57] A. Datta, N. Ganguly, N. Khan, and S. Rakshit, Exploring collider signatures of the inert Higgs doublet model, Phys. Rev. D 95, 015017 (2017).

[58] P. Poulose, S. Sahoo, and K. Sridhar, Exploring the inert doublet model through the dijet plus missing transverse energy channel at the LHC, Phys. Lett. B 765, 300 (2017).

[59] A. Goudelis, B. Herrmann, and O. Stål, Dark matter in the inert doublet model after the discovery of a Higgs-like boson at the LHC, J. High Energy Phys. 09 (2013) 106.

[60] A. Arhrib, Y.-L. S. Tsai, Q. Yuan, and T.-C. Yuan, An updated analysis of inert Higgs doublet model in light of the recent results from LUX, PLANCK, AMS-02 and LHC, J. Cosmol. Astropart. Phys. 06 (2014) 030.

[61] N. Blinov, J. Kozaczuk, D. E. Morrissey, and A. de la Puente, Compressing the inert doublet model, Phys. Rev. D 93, 035020 (2016).

[62] A. Alves, D. A. Camargo, A. G. Dias, R. Longas, C. C. Nishi, and F. S. Queiroz, Collider and dark matter searches in the inert doublet model from Peccei-Quinn symmetry, J. High Energy Phys. 10 (2016) 015.

[63] M. Aoki, S. Kanemura, and H. Yokoya, Reconstruction of inert doublet scalars at the International Linear Collider, Phys. Lett. B 725, 302 (2013).

[64] S. Kanemura, M. Kikuchi, and K. Sakurai, Testing the dark matter scenario in the inert doublet model by future precision measurements of the Higgs boson couplings, Phys. Rev. D 94, 115011 (2016).

[65] A. Belyaev, N. D. Christensen, and A. Pukhov, CalcHEP 3.4 for collider physics within and beyond the Standard Model, Comput. Phys. Commun. 184, 1729 (2013).

[66] A. Semenov, LanHEP: A package for automatic generation of Feynman rules from the Lagrangian, Comput. Phys. Commun. 115, 124 (1998).

[67] A. Semenov, LanHEP: A package for the automatic generation of Feynman rules in field theory. Version 3.0, Comput. Phys. Commun. 180, 431 (2009).

[68] M. Bondarenko et al., Les Houches 2011: Physics at TeV Colliders New Physics Working Group Report, arXiv: 1203.1488.

[69] D. Eriksson, J. Rathsman, and O. Stal, 2HDMC: TwoHiggs-doublet model calculator physics and manual, Comput. Phys. Commun. 181, 189 (2010).

[70] M. E. Peskin and T. Takeuchi, Estimation of oblique electroweak corrections, Phys. Rev. D 46, 381 (1992). 
[71] M. Baak, J. Cuth, J. Haller, A. Hoecker, R. Kogler, K. Munig, M. Schott, and J. Stelzer (Gfitter Group Collaboration), The global electroweak fit at NNLO and prospects for the LHC and ILC, Eur. Phys. J. C 74, 3046 (2014).

[72] E. Lundstrom, M. Gustafsson, and J. Edsjo, The inert doublet model and LEP II limits, Phys. Rev. D 79, 035013 (2009).

[73] G. Belanger, B. Dumont, A. Goudelis, B. Herrmann, S. Kraml, and D. Sengupta, Dilepton constraints in the inert doublet model from Run 1 of the LHC, Phys. Rev. D 91, 115011 (2015).

[74] A. Pierce and J. Thaler, Natural dark matter from an unnatural Higgs boson and new colored particles at the TeV scale, J. High Energy Phys. 08 (2007) 026.

[75] G. Aad et al. (ATLAS, CMS Collaborations), Measurements of the Higgs boson production and decay rates and constraints on its couplings from a combined ATLAS and CMS analysis of the LHC $p p$ collision data at $\sqrt{s}=7$ and $8 \mathrm{TeV}$, J. High Energy Phys. 08 (2016) 045.

[76] G. Aad et al. (ATLAS Collaboration), Search for invisible decays of a Higgs boson using vector-boson fusion in $p p$ collisions at $\sqrt{s}=8 \mathrm{TeV}$ with the ATLAS detector, J. High Energy Phys. 01 (2016) 172.

[77] CMS Collaboration, A combination of searches for the invisible decays of the Higgs boson using the CMS detector, Report No. CMS-PAS-HIG-15-012, 2015.

[78] P. A. R. Ade et al. (Planck Collaboration), Planck 2013 results. XVI. Cosmological parameters, Astron. Astrophys. 571, A16 (2014).

[79] P. A. R. Ade et al. (Planck Collaboration), Planck 2015 results. XIII. Cosmological parameters, Astron. Astrophys. 594, A13 (2016).

[80] G. Hinshaw et al. (WMAP Collaboration), Nine-year Wilkinson Microwave Anisotropy Probe (WMAP) observations: Cosmological parameter results, Astrophys. J. Suppl. Ser. 208, 19 (2013).

[81] G. Belanger, F. Boudjema, A. Pukhov, and A. Semenov, micrOMEGAs_3: A program for calculating dark matter observables, Comput. Phys. Commun. 185, 960 (2014).

[82] G. Belanger, F. Boudjema, A. Pukhov, and A. Semenov, MicrOMEGAs 2.0: A program to calculate the relic density of dark matter in a generic model, Comput. Phys. Commun. 176, 367 (2007).

[83] G. Belanger, F. Boudjema, P. Brun, A. Pukhov, S. RosierLees, P. Salati, and A. Semenov, Indirect search for dark matter with micrOMEGAs2.4, Comput. Phys. Commun. 182, 842 (2011).

[84] D. S. Akerib et al. (LUX Collaboration), First Results from the LUX Dark Matter Experiment at the Sanford Underground Research Facility, Phys. Rev. Lett. 112, 091303 (2014).

[85] M. Ackermann et al. (Fermi-LAT Collaboration), Constraining Dark Matter Models from a Combined Analysis of Milky Way Satellites with the Fermi Large Area Telescope, Phys. Rev. Lett. 107, 241302 (2011).

[86] B. Eiteneuer, A. Goudelis, and J. Heisig, The inert doublet model in the light of Fermi-LAT gamma-ray data: A global fit analysis, Eur. Phys. J. C 77, 624 (2017).

[87] L. Goodenough and D. Hooper, Possible evidence for dark matter annihilation in the inner Milky Way from the Fermi Gamma Ray Space Telescope, arXiv:0910.2998.
[88] L. J. Hall, K. Jedamzik, J. March-Russell, and S. M. West, Freeze-in production of FIMP dark matter, J. High Energy Phys. 03 (2010) 080.

[89] V. Khachatryan et al. (CMS Collaboration), Search for disappearing tracks in proton-proton collisions at $\sqrt{s}=8 \mathrm{TeV}$, J. High Energy Phys. 01 (2015) 096.

[90] R. D. Ball et al., Parton distributions with LHC data, Nucl. Phys. B867, 244 (2013).

[91] E. Diehl (ATLAS Collaboration), The search for dark matter using monojets and monophotons with the ATLAS detector, AIP Conf. Proc. 1604, 324 (2014).

[92] S. Chatrchyan et al. (CMS Collaboration), Search for dark matter and large extra dimensions in monojet events in $p p$ collisions at $\sqrt{s}=7 \mathrm{TeV}$, J. High Energy Phys. 09 (2012) 094.

[93] V. Khachatryan et al. (CMS Collaboration), Search for dark matter, extra dimensions, and unparticles in monojet events in proton-proton collisions at $\sqrt{s}=8 \mathrm{TeV}$, Eur. Phys. J. C 75, 235 (2015).

[94] M. Drees, H. Dreiner, D. Schmeier, J. Tattersall, and J. S. Kim, CheckMATE: Confronting your favourite new physics model with LHC data, Comput. Phys. Commun. 187, 227 (2015).

[95] J. de Favereau, C. Delaere, P. Demin, A. Giammanco, V. Lemaître, A. Mertens, and M. Selvaggi (DELPHES 3 Collaboration), DELPHES 3, A modular framework for fast simulation of a generic collider experiment, J. High Energy Phys. 02 (2014) 057.

[96] M. Cacciari, G. P. Salam, and G. Soyez, FastJet user manual, Eur. Phys. J. C 72, 1896 (2012).

[97] M. Cacciari and G. P. Salam, Dispelling the $N^{3}$ myth for the $k t$ jet-finder, Phys. Lett. B 641, 57 (2006).

[98] M. Cacciari, G. P. Salam, and G. Soyez, The anti- $k(t)$ jet clustering algorithm, J. High Energy Phys. 04 (2008) 063.

[99] A.L. Read, Presentation of search results: The CL(s) technique, J. Phys. G 28, 2693 (2002).

[100] C. G. Lester and D. J. Summers, Measuring masses of semi-invisibly decaying particles pair produced at hadron colliders, Phys. Lett. B 463, 99 (1999).

[101] A. Barr, C. Lester, and P. Stephens, m(T2): The truth behind the glamour, J. Phys. G 29, 2343 (2003).

[102] H.-C. Cheng and Z. Han, Minimal kinematic constraints and m(T2), J. High Energy Phys. 12 (2008) 063.

[103] T. Sjostrand, S. Mrenna, and P. Z. Skands, PYTHIA 6.4 physics and manual, J. High Energy Phys. 05 (2006) 026.

[104] ATLAS Collaboration, Search for new phenomena in monojet plus missing transverse momentum final states using $10 \mathrm{fb}^{-1}$ of $p p$ Collisions at $\sqrt{s}=8 \mathrm{TeV}$ with the ATLAS detector at the LHC, Report No. ATLAS-CONF2012-147, 2012.

[105] G. Aad et al. (ATLAS Collaboration), Search for pairproduced third-generation squarks decaying via charm quarks or in compressed supersymmetric scenarios in $p p$ collisions at $\sqrt{s}=8 \mathrm{TeV}$ with the ATLAS detector, Phys. Rev. D 90, 052008 (2014).

[106] G. Aad et al. (ATLAS Collaboration), Search for new phenomena in final states with an energetic jet and large missing transverse momentum in $p p$ collisions at $\sqrt{s}=$ $8 \mathrm{TeV}$ with the ATLAS detector, Eur. Phys. J. C 75, 299 (2015); Erratum, Eur. Phys. J. C 75, 408 (2015). 